\title{
Pollen-based biome reconstructions for Latin America at 0, 6000 and 18000 radiocarbon years ago
}

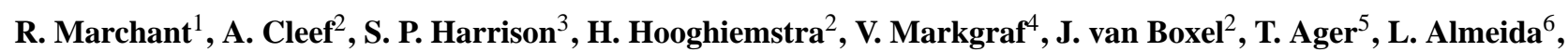

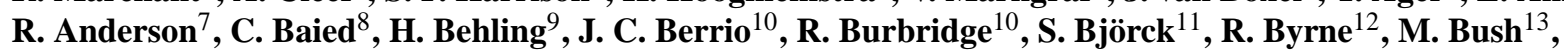
J. Duivenvoorden ${ }^{2}$, J. Flenley ${ }^{14}$, P. De Oliveira ${ }^{15}$, B. van Geel ${ }^{2}$, K. Graf $^{16}$, W. D. Gosling ${ }^{17}$, S. Harbele ${ }^{18}$, T. van der Hammen ${ }^{19}$, B. Hansen ${ }^{20}$, S. Horn ${ }^{21}$, P. Kuhry ${ }^{22}$, M.-P. Ledru ${ }^{23}$, F. Mayle ${ }^{24}$, B. Leyden ${ }^{25}$, S. Lozano-García ${ }^{26}$, A. M. Melief ${ }^{27}$, P. Moreno ${ }^{28}$, N. T. Moar ${ }^{29}$, A. Prieto ${ }^{30}$, G. van Reenen ${ }^{2}$, M. Salgado-Labouriau ${ }^{31}$, F. Schäbitz ${ }^{32}$, E. J. Schreve-Brinkman ${ }^{33}$, and M. Wille ${ }^{34}$

${ }^{1}$ The York Institute for Tropical Ecosystem Dynamics (KITE), Environment Department, University of York, York,

Heslington, YO10 5DD, UK

${ }^{2}$ Institute for Biodiversity and Ecosystem Dynamics (IBED), Faculty of Science, University of Amsterdam, Postbus 94062, 1090 GB Amsterdam, The Netherlands

${ }^{3}$ Bristol Research Initiative for the Dynamic Global Environment (BRIDGE), School of Geographical Sciences, University Road, University of Bristol, Bristol BS8 1SS, UK

${ }^{4}$ INSTAAR, University of Colorado, Boulder, CO 80309, USA

${ }^{5}$ USGS, National Centre, MS 970, Reston, VA 22092, USA

${ }^{6}$ Laboratorio Biogeografía, Facultad de Ciencias, Universidad Nacional Autónoma de México, Aptdo Postal 70-296, 04510

México D.F., Mexico

${ }^{7}$ Department of Geography, University of Montana, Missoula, Montana 59812-1018, USA

${ }^{8}$ Environmental Studies Program, University of Montana, Missoula Montana 59812, USA

${ }^{9}$ Georg-August-Universität, Albrecht-von-Haller-Institut für Pflanzenwissenschaften, Abteilung Palynologie und Klimadynamik, Untere Karspüle 2, 37073 Göttingen, Germany

${ }^{10}$ Department of Geography, University Road, University of Leicester, LE1 7RH, UK

${ }^{11}$ Geological Institute, University of Copenhagen, Øster Volgade 10, 1350 Copenhagen, Denmark

${ }^{12}$ Department of Geography, University of California, Berkeley, California 94720-4740, USA

${ }^{13}$ Dept. of Biological Sciences, Florida Institute of Technology, 150 W. University Boulevard, Melbourne, FL 32905, USA

${ }^{14}$ Department of Geography, Massey University, Palmerston, New Zealand

${ }^{15}$ Instituto de Geociencias-DPE, Universidade de São Paulo, Caixa Postal 11348, São Paulo, SP 05422-970, Brazil

${ }^{16}$ Geographisches Institut der Universität, Winterthürerstraße 190, 8057 Zürich, Switzerland

${ }^{17}$ Dept. of Earth and Environmental Sciences, CEPSA, The Open University, Walton Hall, Milton Keynes, MK7 6AA, UK

${ }^{18}$ Department of Archaeology and Natural History, College of Asia and the Pacific, Australian National University, Canberra, ACT 0200, Australia

${ }^{19}$ Fundación Tropenbos Colombia, Carrera 21 \# 39-35, Santafe de Bogotá, Colombia

${ }^{20}$ Limnological Research Centre, University of Minnesota, 220 Pillsbury Hall, 310 Pillsbury Drive, Minneapolis, MN 55455-0219, USA

${ }^{21}$ Arctic Centre, University of Lapland, PL 122, 96101 Rovaniemi, Finland

${ }^{22}$ Department of Geography, University of Tennessee, 408 G\&G Building, Knoxville, TN 37996-1420, USA

${ }^{23}$ Equipe Paléoenvironements, Institut des Sciences de l'Evolution Institut de Recherche pour le Developement, Montpellier, France

${ }^{24}$ Geography Building, Drummond Street, Edinburgh, EH8 9XP, UK

${ }^{25}$ Department of Geology, University of South Florida, 4202 East Fowler Avenue, SCA 203, Tampa, FL 33620-5200, USA

${ }^{26}$ Universidad Nacional Autónoma de México, Instituto de Geología, Aptdo Postal 70-296, 04510 México D.F., Mexico

${ }^{27} 2$ E Oosterparkstraat 163A, 1092 BE Amsterdam, The Netherlands

${ }^{28}$ Facultad de Ciencias, Universidad de Chile, Casilla 653, Santiago, Chile

${ }^{29}$ Botany Division, D.S.I.R., Private Bag, Christchurch, New Zealand

${ }^{30}$ Laboratorio de Palinologia, National Universidad Mar del Plata, Dept. de Biologia, Funes 3250, 7600 Mar del Plata,

Argentina

${ }^{31}$ Inst. de Geociencias, Fundação Universidade do Brazilia, Campus Universitario, Asa Norte, 0910-900, DF Brazilia, Brazil 


\author{
${ }^{32}$ Geographisches Institut, Universität Bamberg, Am Kranen 1, 96045 Bamberg, Germany \\ ${ }^{33}$ Welbergsweg 8, 7495 SZ Delden, The Netherlands \\ ${ }^{34}$ Geographisches Institut, Universität Essen, Essen, Germany
}

Received: 13 October 2008 - Published in Clim. Past Discuss.: 10 February 2009

Revised: 7 August 2009 - Accepted: 29 September 2009 - Published: 1 December 2009

Abstract. The biomisation method is used to reconstruct Latin American vegetation at $6000 \pm 500$ and $18000 \pm 1000$ radiocarbon years before present $\left({ }^{14} \mathrm{C}\right.$ yr BP) from pollen data. Tests using modern pollen data from 381 samples derived from 287 locations broadly reproduce potential natural vegetation. The strong temperature gradient associated with the Andes is recorded by a transition from high altitude cool grass/shrubland and cool mixed forest to mid-altitude cool temperate rain forest, to tropical dry, seasonal and rain forest at low altitudes. Reconstructed biomes from a number of sites do not match the potential vegetation due to local factors such as human impact, methodological artefacts and mechanisms of pollen representivity of the parent vegetation.

At $6000 \pm 500{ }^{14} \mathrm{C}$ yr BP 255 samples are analysed from 127 sites. Differences between the modern and the $6000 \pm 500{ }^{14} \mathrm{C}$ yr BP reconstruction are comparatively small; change relative to the modern reconstruction are mainly to biomes characteristic of drier climate in the north of the region with a slight more mesic shift in the south. Cool temperate rain forest remains dominant in western South America. In northwestern South America a number of sites record transitions from tropical seasonal forest to tropical dry forest and tropical rain forest to tropical seasonal forest. Sites in Central America show a change in biome assignment, but to more mesic vegetation, indicative of greater plant available moisture, e.g. on the Yucatán peninsula sites record warm evergreen forest, replacing tropical dry forest and warm mixed forest presently recorded.

At $18000 \pm 1000{ }^{14} \mathrm{C}$ yr BP 61 samples from 34 sites record vegetation reflecting a generally cool and dry environment. Cool grass/shrubland is prevalent in southeast Brazil whereas Amazonian sites record tropical dry forest, warm temperate rain forest and tropical seasonal forest. Southernmost South America is dominated by cool grass/shrubland, a single site retains cool temperate rain forest indicating that forest was present at some locations at the LGM. Some sites in Central Mexico and lowland Colombia remain unchanged in the biome assignments of warm mixed forest and tropical dry forest respectively, although the affinities that these sites have to different biomes do change between $18000 \pm 1000$

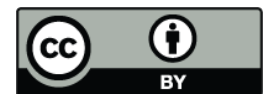

Correspondence to: R. Marchant (rm524@york.ac.uk)
${ }^{14} \mathrm{C}$ yr BP and present. The "unresponsive" nature of these sites results from their location and the impact of local edaphic influence.

\section{Introduction}

Biomisation is an objective method to reconstruct broad vegetation types based on the assignment of pollen taxa to one or more plant functional types (PFTs) (Prentice et al., 1996a). The method is based on the assumption that a pollen spectrum will have different degrees of affinity to different biomes that can be quantified by a simple algorithm. Biome reconstructions from pollen data at $6000 \pm 500{ }^{14} \mathrm{C} \mathrm{yr} \mathrm{BP}$ and the last glacial maximum (LGM) at $18000 \pm 1000{ }^{14} \mathrm{C}$ yr BP have been produced for most regions of the world under the auspices of the BIOME 6000 project (Prentice et al., 1998, 2000). The validity of the method in reconstructing biomes at different time intervals has been demonstrated for Africa (Jolly et al., 1998a; Elenga et al., 2000), Australia (Pickett et al., 2004) Beringia (Bigelow et al., 2003; Edwards et al., 2000), China (Yu et al., 1998, 2001), Eastern North America (Williams et al., 2000), Eurasia (Tarasov et al., 1998a), Europe (Prentice et al., 1996a, b; Tarasov et al., 1998a, b; Elenga et al., 2000), Japan (Takahara et al., 2001) and Western North America (Thompson and Anderson, 2000). Results from Latin America, presented here, represent the last geographically large area to undergo this process. Within Latin America the biomisation method has been previously applied to Colombian and Mexican pollen data at a range of spatial and temporal scales; from the middle Holocene (Marchant et al., 2001a; Ortega-Rosas et al., 2008a, b), the LGM (Marchant et al., 2002a), to investigate modern-pollen vegetation relationships (Marchant et al., 2001b), impact of human societies on vegetation (Marchant et al., 2004a) and as a basis for comparisons with output from a vegetation model run under different climatic and environmental scenarios (Marchant et al., 2004b, 2006). In addition to these spatial investigations, the method has been applied downcore down to a 450000 year pollen record from the high plain of Bogotá (Marchant et al., 2002b). As Colombia is biogeographically complex, encompasses high altitude, temperate and tropical floras reflecting a range of environmental space including transitions from hyper-humid to semi-arid climates, these analyses provided a suitable test-bed for the wider geographical focus presented here. 
In addition to reconstructing vegetation patterns, and investigating factors that can explain observed changes, data on past biomes contributes to testing of climate and vegetation models (Prentice et al., 1992; Haxeltine and Prentice, 1996; Peng et al., 1998; Marchant et al., 2006; Braconnot et al., 2007). Vegetation models can be used to portray output from Global Circulation Models (GCMs) as maps of potential vegetation (Claussen and Esh, 1994; Foley et al., 1996; Prentice et al., 1996b; Williams et al., 1998) that can be used in the development of models that couple biosphere, atmosphere and oceanic components (Braconnot et al., 2007; Claussen, 1994; Harrison et al., 2003; Texier et al., 1997) and testing of biogeochemical dynamics (Peng et al., 1998). There has been growing interest has focused how atmosphere-biosphere interactions have operated under the changing environmental conditions since the LGM, particularly in trying to understand the response of ecosystems to different types of environmental forcing (Jolly and Haxeltine, 1997). Transformed pollen data can further be used in conjunction with other data types, such as on lake status (Jolly et al., 1998b) and archaeological evidence (Piperno et al., 1990, 1991a, b), to better understand the causal factors driving vegetation change over the recent geological past.

\subsection{Latin American region}

Latin America comprises the area from $35^{\circ} \mathrm{N}$ to $65^{\circ} \mathrm{S}$, and from $35^{\circ} \mathrm{W}$ to $120^{\circ} \mathrm{W}$ extending from México to islands off southernmost South America from Eastern Brazil to the Galapagos Islands. Latin America is characterised by strong environmental gradients associated with $100^{\circ}$ of latitude, approximately $7000 \mathrm{~m}$ of altitude and the transition from oceanic- to continentally-dominated climate systems (Fig. 1). Despite this great extent there has been permanent contact between the tropical and temperate domains throughout geological time. Physiographically, Latin America is characterised by stable cratons associated with the interior and areas of active mountain building, particularly associated with the Andes. This environmental variability is reflected by an incredibly diverse biogeography, ranging from the highly diverse rain forest of the Chocó Pacific (Colombia) to the cold deserts of the high Andes, from the hot semidesert areas of México to the cold moorlands of Tierra del Fuego (Fig. 2). Descending an altitudinal gradient there is a transition from páramo (cool grass/shrubland ) to high Andean forests (cool mixed and cool temperate rain forests) and lower Andean forest (warm evergreen forest) (Fig. 3). Complicating this potential vegetation distribution is the factor of human impact with the majority of the vegetation in Latin America being impacted on by the vegetation (Ellis and Ramankutty, 2008). The timing of early human settlement in Latin America is a contentious subject, although it seems from the early Holocene there was considerable cultural diversity and adaptation to a series of different environments (Gnécco, 1999). Human-induced impact has had a direct in-

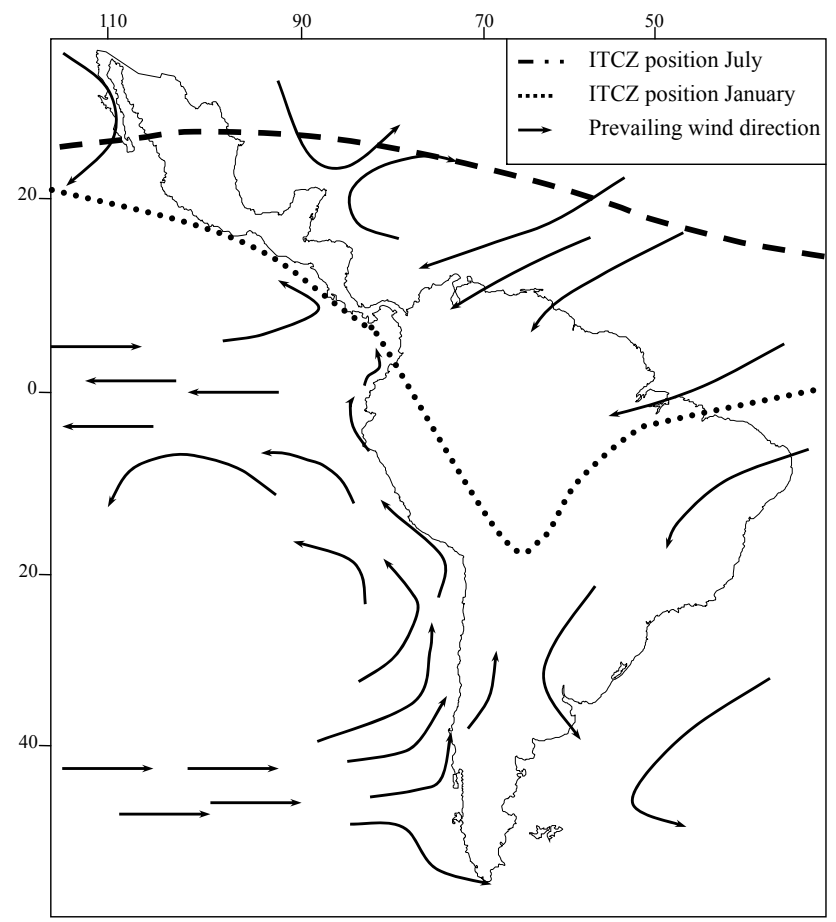

Fig. 1. Map of Latin America depicting the present-day summer and winter position of the ITCZ and the macro-scale wind (and hence moisture) patterns over Latin America.

fluence on vegetation composition and distribution through land-use practices and the introduction of alien taxa and cultivars to the Latin American flora. For example, in excess of 100 plants were under cultivation prior to the European conquests in the 15 th century (Piperno et al., 2000).

\subsection{Latin America climate}

Cerverny (1998), Eidt (1968) and Metcalfe et al. (2000) have reviewed Latin American climate. Given the broad geographical scope, Latin America is characterised by a variety of climates that relates to its global position, shape of the landmass, location and height of the Andes, offshore currents, general hemisphere air flow and proximity of large water bodies (Fig. 1). Four dominant circulation regimes influence Latin America: the Inter-Tropical Convergence Zone (ITCZ), the prevailing westerlies, the semi-permanent high pressure cells located over the South Pacific and South Atlantic Oceans and the trade winds. Perhaps most dominant is the annual oscillation of the meteorological equator (ITCZ), this migrating some $10-15^{\circ}$ latitude about the equator (Fig. 1). The ITCZ reaches its northernmost location in June, this bringing high rainfall for northern South America and the Caribbean, with January and February recording the dry season (Cerveny, 1998). However, due to the influence of the westerlies from the Pacific, and the sharply rising topography of the Andes, the ITCZ has a sinusoidal 


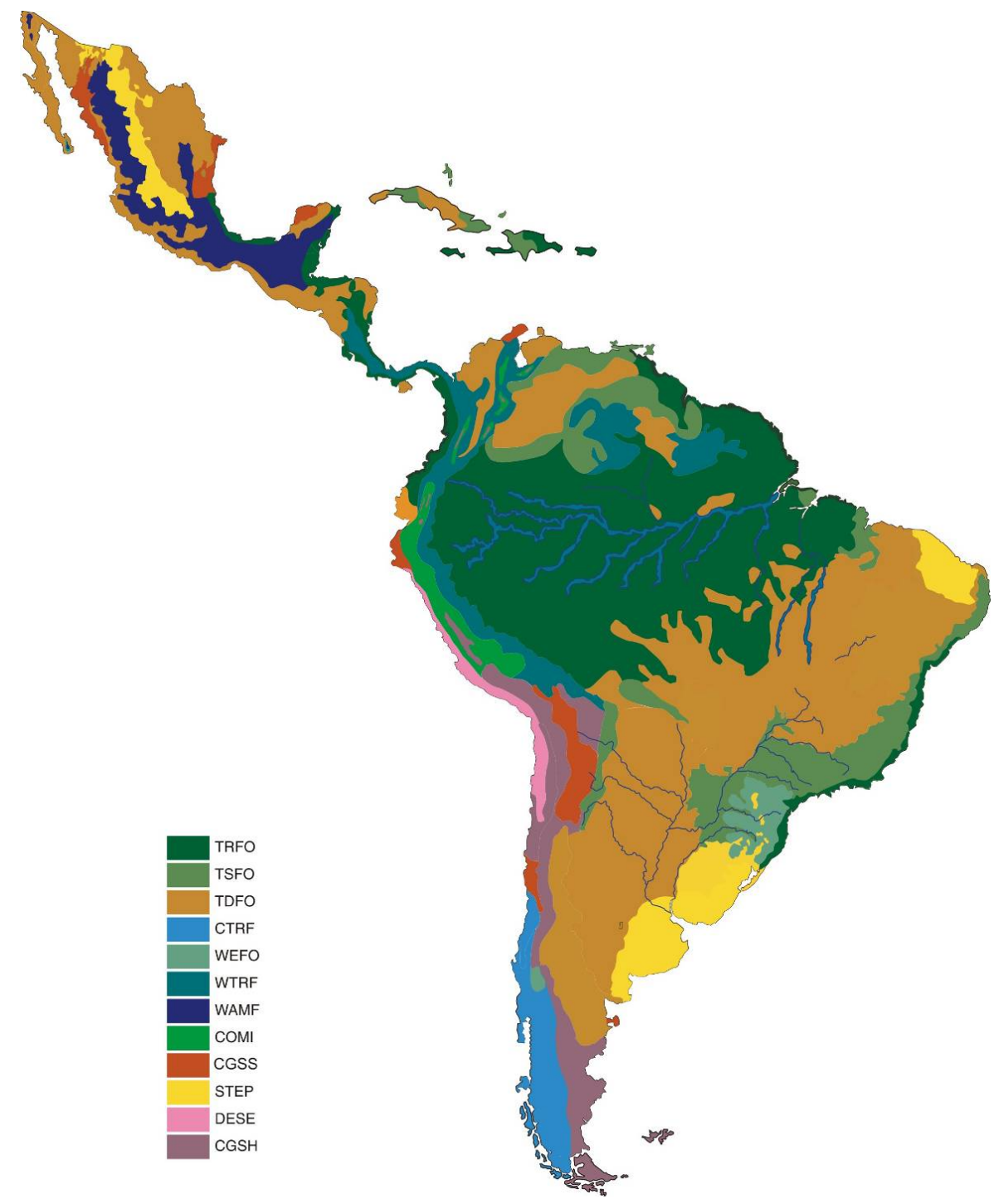

Fig. 2. Map of the modern potential vegetation as derived from Schmithüsen (1976) and Hück (1960). For example, the various divisions of seasonally dry forest such as cerrado, caatinga, campo rupstre, savanna, are combined to the biome of tropical dry forest. Acronyms are explained in Table 1.

profile over northwestern South America (Fig. 1). In southern South America the prevailing westerlies south of $40^{\circ} \mathrm{S}$ are particularly important in controlling the moisture regime. The topographic barrier of the Andes contributes to the creation of two large semi-present anticyclones, one over the South Pacific and one over the South Atlantic, the southeast trade winds associated with this latter system brings abundant moisture to the Amazon Basin (Cerveny, 1998). Due to the large size of South America, and the highland ranges that fringe much of the continent there is often a rapid transition from relatively moist coastal areas to a dry interior reflecting the transition from oceanic- to continental-dominated climate systems. For example, due to the proximal location of the Pacific-based moisture source and steeply rising ground, precipitation is highest ( $>15000 \mathrm{~mm} \mathrm{yr}^{-1}$ ) in the Chocó Pacific region. Exceptions to this scenario are areas located between the anticyclones, e.g. the Peruvian coast, where relatively arid conditions prevail.

One of the main environmental gradients in Latin America is associated with the Andes. The Andes are characterised by a diurnal climate (Kuhry, 1988); at a given location differences in monthly temperature are small $\left(<3^{\circ} \mathrm{C}\right)$ although daily fluctuations may be large $\left(20^{\circ} \mathrm{C}\right)$, especially during the dry seasons. Climatic changes with altitude can be summarised as a lapse rate (Barry and Chorley, 1990). Applying a lapse rate of $6.6^{\circ} \mathrm{C} 1000 \mathrm{~m}^{-1}$ (Van der Hammen and González, 1965; Wille et al., 2001), this altitudinal rise equates to a temperature change of more than $30^{\circ} \mathrm{C}$. Also 

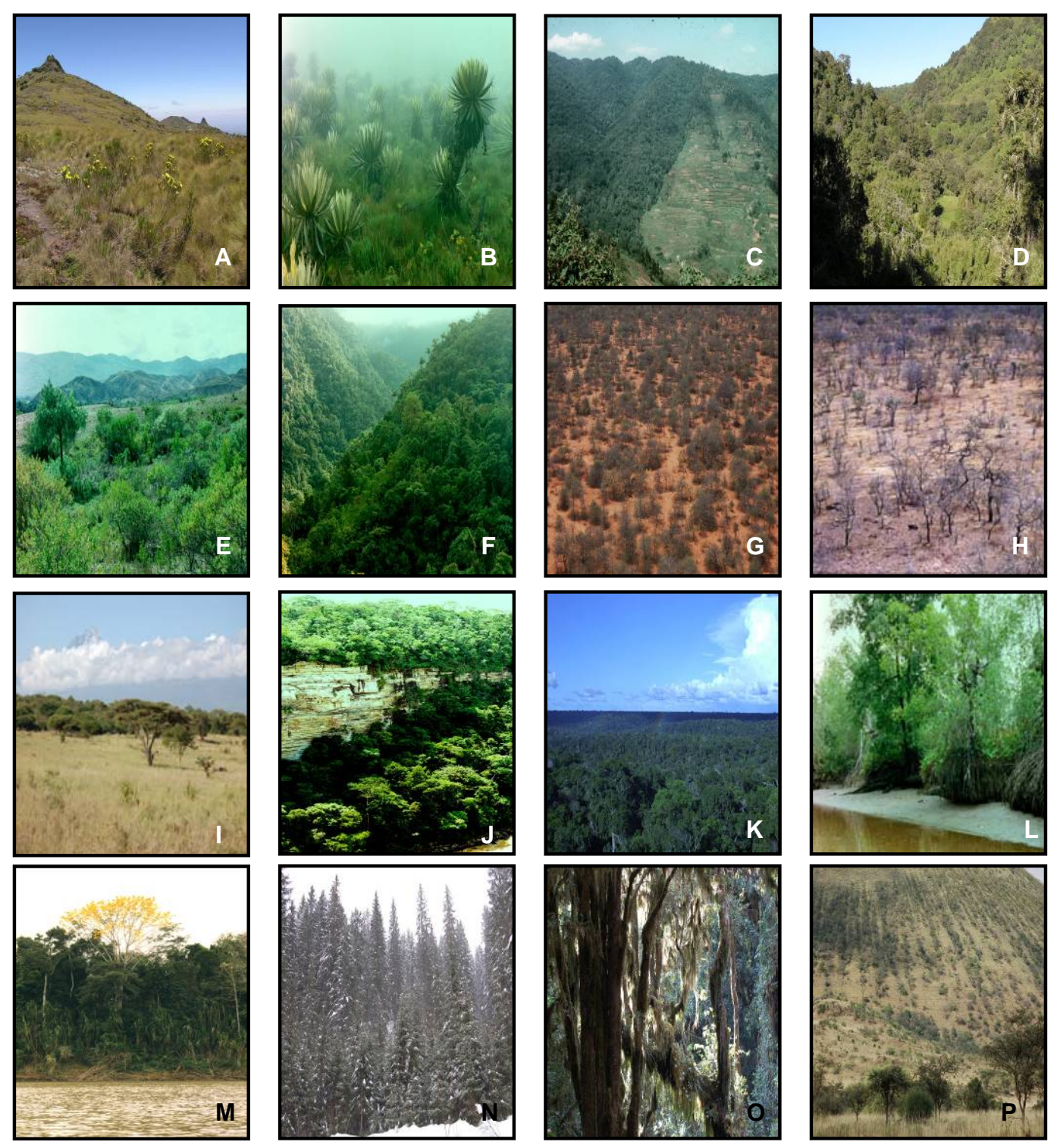

Fig. 3. Examples of Latin America biomes from cool grass shrubland with Scencio and Espeletia (a, b) to cool mixed forest (c, d, e, f), tropical dry forest that can be dominated by Bauhina $(\mathbf{g}, \mathbf{h})$ with dominance of open steppe grasslands (i) tropical rain forest $(\mathbf{j}, \mathbf{k})$, dominated by mangrove in areas fringing saline water (l), tropical seasonal forest (m), cool temperate forest (n, o). The bottom plate (p) shows the importance of edpahic factors on controlling vegetation, in this case local hydrology where rill channels allow trees to grow in areas that would be dominated by grassland.

associated with the Andes are steep gradients of moisture availability. Rainfall is high on the eastern slopes of the Andes; the concave nature acting as a receptacle for moisture transferred by the southeast trade winds from the Atlantic Ocean, in part receiving moisture generated by the Amazonian forest (Fjeldså, 1993). Low rainfall is recorded within rain shadow areas, such as on the lower slopes of the Magdalena Valley and the inter-Andean plains (Kuhry, 1988). These climate gradients result in rapid transitions from mesic to xeric vegetation types, e.g., cool high-altitude grasslands change to "temperate" forests at mid-altitudes and diverse tropical rain forests within a few kilometres (Fig. 3). In the southern part of Latin America rainfall is largely controlled by the persistence and strength of the westerly winds (Gilli et al., 2005). There has been increased interest in largescale temperature-driven surface pressure oscillations in the Pacific Ocean termed the Southern Oscillation, and its assimilated oceanic aspects, El Niño and its antithesis La Niña (Cerveny, 1998; Godínez-Domínguez et al., 2000; Metcalfe et al., 2000), however, given the temporal foci of the vegetation reconstructions here this is not so relevant. 
Table 1. Range of plant functional types identified within the Latin American region giving bioclimatic range and physiological adaptation.

\begin{tabular}{|c|c|c|}
\hline Code & Plant functional type & Bioclimatic range and plant physiological adaption \\
\hline g & Graminoid & $\begin{array}{l}\text { Ecologically broad category that occurs in a number of biomes, a highly adap- } \\
\text { tive PFT with a ubiquitous distribution and little diagnostic value. }\end{array}$ \\
\hline man & Mangrove & $\begin{array}{l}\text { Constituent of lowland tropical vegetation, control on distribution is mainly } \\
\text { hydrological }\end{array}$ \\
\hline tx & Tree fern & $\begin{array}{l}\text { Can be locally dominant. Occupying a broad bioclimatic range, particularly } \\
\text { common in temperate moist areas. }\end{array}$ \\
\hline $\mathrm{Te}_{1}$ & Tropical broad-leaved evergreen tree & MTCO $>15.5^{\circ} \mathrm{C}, \alpha>0.7$, short dry season (1month), GDD $>5000$ \\
\hline $\mathrm{Te}_{2}$ & Tropical xeric broad-leaved evergreen tree & $\begin{array}{l}\text { MTCO }>15.5^{\circ} \mathrm{C}, \alpha>0.6-08 \text {, longer dry season ( } 2-4 \text { months), GDD }>5000 \text {, } \\
\text { withstands longer dry season by shedding leaves. }\end{array}$ \\
\hline $\operatorname{Tr}_{1}$ & Tropical rain green tree & MTCO $>15.5^{\circ} \mathrm{C}, \alpha>0.9, \mathrm{GDD}>5000$, present in wettest tropical rain forest. \\
\hline $\operatorname{Tr}_{2}$ & Dry tropical rain green tree & $\begin{array}{l}\text { MTCO }>15.5^{\circ} \mathrm{C}, \alpha 0.8-0.9, \mathrm{GDD}>5000 \text {, present in range of tropical seasonal } \\
\text { forest types. }\end{array}$ \\
\hline ctc & Cold temperate conifer & MTCO $5^{\circ} \mathrm{C}-15^{\circ} \mathrm{C}, \alpha>0.7$, GDD $>4500$, common in the Brazilian highlands. \\
\hline $\operatorname{ctc}_{1}$ & Cool temperate conifer & $\begin{array}{l}\text { MTCO }-5^{\circ} \mathrm{C}-10^{\circ} \mathrm{C}, \alpha 0.95-0.75, \mathrm{GDD}>900, \text { common along the western } \\
\text { coast of southern South America. }\end{array}$ \\
\hline $\operatorname{ctc}_{2}$ & Maritime evergreen conifer & $\begin{array}{l}\text { MTCO }-10^{\circ} \mathrm{C}-5^{\circ} \mathrm{C}, \alpha>0.65, \mathrm{GDD}>1000 \text {, common along the western coast } \\
\text { of southern South America. }\end{array}$ \\
\hline ec & Eurythermic conifer & $\begin{array}{l}\mathrm{MTCO}>5^{\circ} \mathrm{C}, \alpha 0.4-0.6, \mathrm{GDD}>5000 \text {, common within dry forest of South } \\
\text { America and Mexico. }\end{array}$ \\
\hline txts & Tropical xerophytic tree/shrub & MTCO $>20^{\circ} \mathrm{C}, \alpha 0.2-0.35$, GDD $>5000$, woody shrubs common in dry forest. \\
\hline ds & Desert shrub & $\begin{array}{l}\text { MTCO }>20^{\circ} \mathrm{C}, \alpha 0.2-0.35, \mathrm{GDD}>5000 \text {, woody shrub and cacti in Mexico } \\
\text { and coastal Peru }\end{array}$ \\
\hline df & Xerophytic forb & $\begin{array}{l}\text { MTCO }>20^{\circ} \mathrm{C}, \alpha 0.2-0.35, \mathrm{GDD}>5000 \text {, woody shrub and cacti in Mexico } \\
\text { and coastal Peru }\end{array}$ \\
\hline $\mathrm{tf}$ & Tropical forb & $\mathrm{MTCO}>15.5^{\circ} \mathrm{C}, \alpha>0.6, \mathrm{GDD}>5000$, frost intolerant \\
\hline tef & Temperate forb & $\mathrm{MTCO}>5^{\circ} \mathrm{C}-15^{\circ} \mathrm{C}, \alpha>0.6, \mathrm{GDD}>1000$, frost tolerant \\
\hline sf & Eurythermic forb & $\begin{array}{l}\text { MTCO } 5^{\circ} \mathrm{C}-10^{\circ} \mathrm{C}, \alpha 0.65-0.7, \text { GDD } 2500-4000 \text {, requires a seasonal moist } \\
\text { environment }\end{array}$ \\
\hline af & Arctic forb & MTCO $-5^{\circ} \mathrm{C}-0^{\circ} \mathrm{C}, \alpha 0.05-0.1, \mathrm{GDD}<500$, frost tolerant \\
\hline $\mathrm{cp}$ & Cushion forb & MTCO $<-5^{\circ} \mathrm{C}, \alpha<0.2$, GDD $<500$, specific growth form, frost tolerant. \\
\hline wte & Warm temperate evergreen broad-leaved tree & MTCO $5^{\circ} \mathrm{C}-15^{\circ} \mathrm{C}, \alpha>0.65, \mathrm{GDD}>3000$, frost tolerant mesophyllous trees \\
\hline ts & Temperate summer green tree & $\begin{array}{l}\text { MTCO } 0^{\circ} \mathrm{C}-5^{\circ} \mathrm{C}, \alpha>0.65, \mathrm{GDD}>2000 \text {, frost tolerant micro and mesophyl- } \\
\text { lous trees }\end{array}$ \\
\hline $\mathrm{ts}_{1}$ & Temperate evergreen broad-leaved tree & MTCO $-5^{\circ} \mathrm{C}-5^{\circ} \mathrm{C}, \alpha>0.5$, GDD $>1000$, sclerophyllous, usually evergreen \\
\hline wte $_{1}$ & Temperate cool deciduous broad-leaved tree & $\begin{array}{l}\text { MTCO } 0^{\circ} \mathrm{C}-15^{\circ} \mathrm{C}, \alpha>0.6, \mathrm{GDD}>3000 \text {, winter deciduous, requires warm } \\
\text { growing season. }\end{array}$ \\
\hline wte $_{4}$ & Temperate cold-deciduous broad-leaved tree & $\begin{array}{l}\text { MTCO }-5^{\circ} \mathrm{C}-5^{\circ} \mathrm{C}, \alpha 0.55 \text {, GDD is }>2500 \text {, winter deciduous, requires warm } \\
\text { growing season but this can be short. }\end{array}$ \\
\hline aa & Arctic shrub & MTCO $-5^{\circ} \mathrm{C}-0^{\circ} \mathrm{C}, \alpha 0.2-0.4$, GDD 500-1000, frost tolerant \\
\hline
\end{tabular}



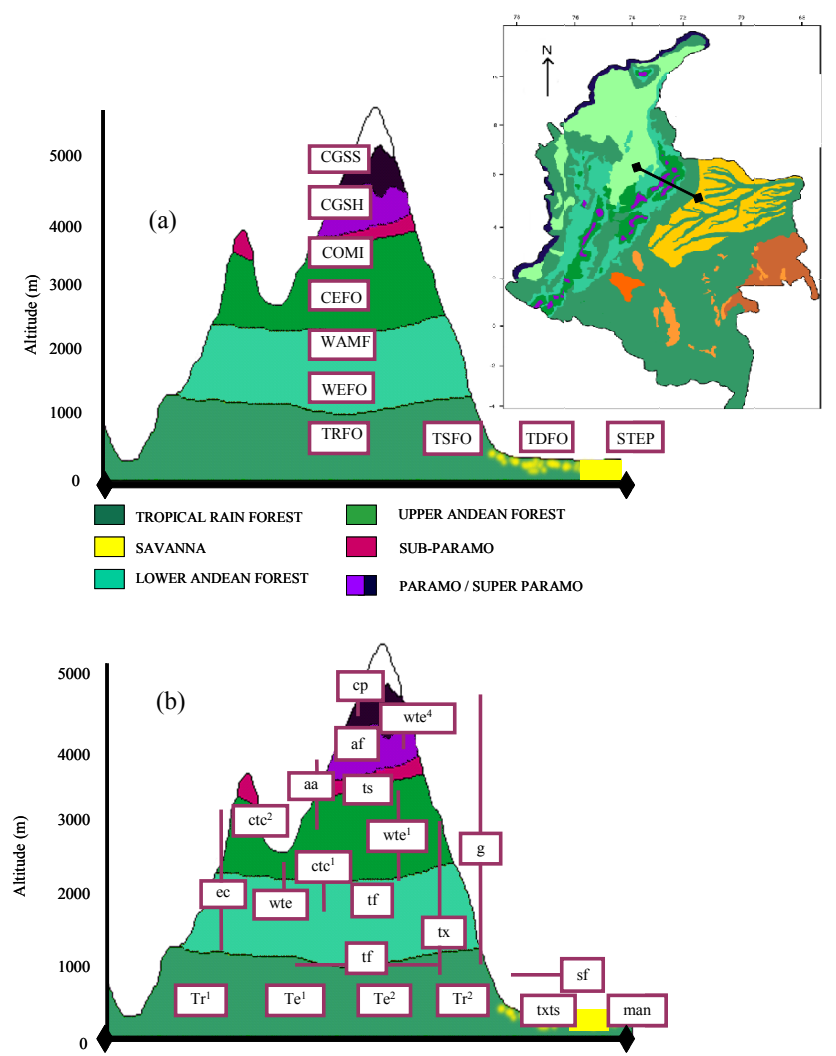

Fig. 4. Cross an altitudinal cross section across the northern Andes in Colombia showing the standard vegetation units and their relationship to Biomes (a) and plant functional types (b). Acronyms are explained in Tables 1 and 2 .

\subsection{Latin America vegetation}

For the purpose of this investigation the potential vegetation composition and distribution Latin America is classified at a coarse resolution with twelve biomes being identified (Fig. 2) that summarise the 57 categories mapped by Hück (1960) and 45 by Schmithüsen (1976). This process of reclassifying the vegetation was carried out in consultation with a number of the co-authors, particularly those with good ecological knowledge and a range of geographical expertise. Reducing the number of vegetation categories results in a significant smoothing of the ecosystem transitions, particularly when these are very sharp or specific biomes are quite isolated. A good example are the relatively small deserts of north-western Mexico that get mapped as tropical dry forest rather than desert. The vegetation composition and distribution generally reflects the main climatic and topographic gradients described above. However, a series of caveats to this must be stressed. Firstly, the actual and potential vegetation can be quite different, the former reflecting a long history of human interaction that has been particularly pronounced since the colonial period but has been influencing the vegetation for at least the last 5000 years (Marchant et al., 2004). In numerous areas this interaction has completely transformed the potential vegetation to an agricultural landscape. Another factor complicating the relationship between climate and vegetation is the locally strong edaphic influence by substrate, topography or geographic character (Fig. 3). The strength of this influence is demonstrated by areas of tropical dry forest that form on free-draining sandstones, e.g. the Llanos Orientales (Colombia); these are located in areas where the climate regime would support tropical seasonal forest, or even tropical rain forest.

Broad types of vegetation with similar composition and distribution (biomes) result from a combination of plant functional types (PFTs). PFTs and biomes, which lie at the heart of the biomisation technique, allow the high floristic diversity within the Latin America pollen flora to link with the relatively coarse vegetation classification (Fig. 4). PFTs group together species that have common character (Prentice et al., 1992). This grouping is based on common life form and phenology, combined with the geographic distribution that is in part determined by climate (Woodward, 1987). An indication of the bioclimatic range of each PFT and plant physiological adaptation, to the given environmental condition, is presented in Table 1. The range of biomes identified within the Latin America, floristic description, main location and equivalent floristic units is portrayed in Table 2. The cool grass/shrubland biome incorporates a relatively wide range of vegetation dominated by grasses, heath, cool temperate sclerophyll shrubs and cushion plants (Fig. 3). This biome is present in southern South America and at high altitudes along the Andes. In addition to the cool grassland a warm grassland (steppe) is identified. Steppe is found predominately under the warm, dry climates of southeast and northeast Brazil, northwestern Argentina and coastal northern South America. Warm temperate rain forest represents a mix of warm conifers such as Araucaria, Andean and Atlantic rain forest elements, whereas cool temperate rain forest contains cool conifers, such as Fitzroya, Andean and Valdivian rain forest elements. Dry forests are extensive in Latin America, specifically associated with areas located between the two semi-permanent anticyclones and influenced by the high seasonality of rainfall imposed by the annual migration of the ITCZ. For our classification we characterise the diverse dry vegetation formations (Fig. 4) as the tropical dry forest biome. Xerophytic trees and shrubs is widespread in the interior of South America, along the southwestern Pacific coast and northeast Brazil where it grades into steppe, additionally, there are patches in Colombia, on the Yucatán peninsula and in México (Fig. 2). Tropical dry forest is predominantly recorded in two main swaths either side of the Amazon basin, with an extension through Central America. The tropical seasonal forest biome is predominantly recorded to the north of Amazonia where it is interspersed with patches of dry forest; this reflecting a strong edaphic influence. A large area of tropical seasonal forest is recorded away from the hyperhumid area of Brazil along the Atlantic coast. The tropical 
Table 2. Biomes identified within the Latin American region as portrayed in the vegetation map (Fig. 2) indicting a floristic description, the main location and equivalent floristic units found in a macro scale analysis of the Latin American vegetation.

\begin{tabular}{|c|c|c|c|c|c|}
\hline Code & Biome & Definition & Main locations & Equivalent & Floristic characteristics \\
\hline TRFO & $\begin{array}{l}\text { Tropical } \\
\text { rainforest }\end{array}$ & $\begin{array}{l}\text { Closed canopy low- } \\
\text { land evergreen forests. } \\
\text { Canopy broken by } \\
\text { emergent trees }(>40 \mathrm{~m}) \text {. } \\
\text { MTCO }>18^{\circ} \mathrm{C} \text {, precipi- } \\
\text { tation }>1500 \mathrm{~mm} \mathrm{yr}^{-1} \text {, } \\
\text { frost-intolerant. }\end{array}$ & $\begin{array}{l}\text { Characterise much of } \\
\text { the Amazon catchment. } \\
\text { Can form a relatively } \\
\text { thin band along tropi- } \\
\text { cal coastal areas, e.g. } \\
\text { Atlantic rain forest of } \\
\text { Brazil, Chocó pluvial } \\
\text { forest of Colombia, } \\
\text { maintained by high } \\
\text { moisture derived from } \\
\text { close proximity of } \\
\text { oceanic influence. }\end{array}$ & $\begin{array}{l}\text { Amazonian forest, Trop- } \\
\text { ical moist forest, Atlantic } \\
\text { rain forest, Terra firme } \\
\text { forest, Várzea, Gallery } \\
\text { forest, Chocó pluvial for- } \\
\text { est. }\end{array}$ & $\begin{array}{l}\text { Generally characterised } \\
\text { by plants with meso- } \\
\text { phyll leaf, although some } \\
\text { sclerophyllous plants are } \\
\text { present, often tree ferns } \\
\text { and palms. }\end{array}$ \\
\hline TSFO & $\begin{array}{l}\text { Tropical } \\
\text { seasonal } \\
\text { forest }\end{array}$ & $\begin{array}{l}\text { Relatively tall }(20-30 \mathrm{~m}) \\
\text { closed canopy forest } \\
\text { with occasionally tall } \\
(>40 \mathrm{~m}) \text { emergent trees. } \\
\text { Canopy opens in a } \\
\text { mosaic as deciduous } \\
\text { elements loose leaves. } \\
\text { Seasonally dry from } 1-4 \\
\text { months. }\end{array}$ & $\begin{array}{l}\text { Dominant to the north of } \\
\text { Amazonian tropical rain- } \\
\text { forest, in central Amer- } \\
\text { ica and formerly exten- } \\
\text { sive in the interior of } \\
\text { Brazil prior to extensive } \\
\text { clearance. }\end{array}$ & $\begin{array}{l}\text { Marsh forests, savanna } \\
\text { gallery forest, Seasonal } \\
\text { swamp forest with } \\
\text { palms. }\end{array}$ & $\begin{array}{l}\text { A mix of mesophyllous } \\
\text { and sclerophyllous taxa } \\
\text { The structure of the for- } \\
\text { est is dependent on mois- } \\
\text { ture demand and length } \\
\text { of dry season - this de- } \\
\text { termines the amount of } \\
\text { deciduous taxa. Palms } \\
\text { can be locally common. }\end{array}$ \\
\hline TDFO & $\begin{array}{l}\text { Tropical dry } \\
\text { forest }\end{array}$ & $\begin{array}{l}\text { Relatively low }(5-10 \mathrm{~m}) \text {, } \\
\text { occasionally tall }(20 \mathrm{~m}) \\
\text { trees. Mixed forest, } \\
\text { forming where the dry } \\
\text { season leads to drought } \\
\text { and plant water stress. }\end{array}$ & $\begin{array}{l}\text { Extensive in central } \\
\text { Brazil and central South } \\
\text { America adjacent trop- } \\
\text { ical rainforest. More } \\
\text { fragmented in north- } \\
\text { western South America } \\
\text { where free draining } \\
\text { substrate leads to water- } \\
\text { stress. Extends to mid } \\
\text { altitudes, particularly } \\
\text { within rain shadow } \\
\text { areas. Extensive in } \\
\text { western Central America } \\
\text { and Mexico. Present on } \\
\text { the Galápagos Islands. }\end{array}$ & $\begin{array}{l}\text { Andean xerophytic bush, } \\
\text { Cerrado, Campo ru- } \\
\text { pestres, Campo cerrado } \\
\text { ("campo" is more asso- } \\
\text { ciated with grasslands). } \\
\text { Cactus forest, Matorral, } \\
\text { Deciduous xerophytic } \\
\text { forest, Andean xero- } \\
\text { phytic bush, Espinar, } \\
\text { Restinga dune forests, } \\
\text { Thorn forest, Chaco. }\end{array}$ & $\begin{array}{l}\text { Xeromorphic charac- } \\
\text { teristic, particularly fire } \\
\text { tolerant. For example, } \\
\text { microphyllous leaves, } \\
\text { thorns, deciduous leaves, } \\
\text { thick bark, stomata often } \\
\text { present along lines } \\
\text { Drought adapted taxa } \\
\text { are common, e.g. tree } \\
\text { cacti (Opuntia and Jas } \\
\text { minocerus) with dense } \\
\text { undergrowth of shrubs } \\
\text { and herbs. }\end{array}$ \\
\hline WTRF & $\begin{array}{l}\text { Warm tem- } \\
\text { perate rain- } \\
\text { forest }\end{array}$ & $\begin{array}{l}\text { Evergreen closed forest, } \\
\text { of relatively low stature } \\
(<20 \mathrm{~m}) \text { with tall emer- } \\
\text { gent trees }(>25 \mathrm{~m}) \text {. Not } \\
\text { tolerant of freezing. A } \\
\text { transitional forest type } \\
\text { between lowland and } \\
\text { higher altitude forms } \\
(1000-2500 \mathrm{~m}) \text {. }\end{array}$ & $\begin{array}{l}\text { Extending along the } \\
\text { Andes at mid to low el- } \\
\text { evations }(500-2000 \mathrm{~m}) \text {. } \\
\text { Present at slightly lower } \\
\text { elevations in eastern } \\
\text { Brazilian highlands } \\
(<1000 \mathrm{~m}) \text {. }\end{array}$ & $\begin{array}{l}\text { Lower montane forest, } \\
\text { Moist lower montane } \\
\text { forest, Submontane } \\
\text { forest and Araucaria- } \\
\text { dominated forest also } \\
\text { with Podocarpus. }\end{array}$ & $\begin{array}{l}\text { A mix of mesophyllous } \\
\text { and sclerophyllous taxa } \\
\text { constrained by altitude } \\
\text { and length of dry season } \\
\text { Palms and tree ferns can } \\
\text { be locally common. }\end{array}$ \\
\hline WEFO & $\begin{array}{l}\text { Warm- } \\
\text { temperate } \\
\text { evergreen } \\
\text { broadleaf } \\
\text { forest }\end{array}$ & $\begin{array}{l}\text { Evergreen semi-closed } \\
\text { forest with tall emergent } \\
\text { trees }(>30 \mathrm{~m}) \text {. Not } \\
\text { tolerant of freezing. }\end{array}$ & $\begin{array}{l}\text { Present within a rel- } \\
\text { atively restricted range } \\
\text { and along the Andes, } \\
\text { particularly present from } \\
1000-2000 \mathrm{~m} \text {. }\end{array}$ & $\begin{array}{l}\text { Andean forest, Transi- } \\
\text { tional Andean forest, } \\
\text { Upper Andean forest. }\end{array}$ & $\begin{array}{l}\text { A mix of mesophyllous } \\
\text { and sclerophyllous taxa } \\
\text { Tree ferns can be locally } \\
\text { common. }\end{array}$ \\
\hline
\end{tabular}


Table 2. Continued.

\begin{tabular}{|c|c|c|c|c|c|}
\hline Code & Biome & Definition & Main locations & Equivalent & Floristic characteristics \\
\hline WAMF & $\begin{array}{l}\text { Warm } \\
\text { temperate } \\
\text { mixed forest }\end{array}$ & $\begin{array}{l}\text { Medium height }(<15 \mathrm{~m}) \\
\text { open canopy with open } \\
\text { under-story. Drought tol- } \\
\text { erant, semi fire-tolerant. }\end{array}$ & $\begin{array}{l}\text { Mid to high altitudes of } \\
\text { north Central America, } \\
\text { in particular Mexico. }\end{array}$ & $\begin{array}{l}\text { Pinus and Quercus- } \\
\text { dominated forest. }\end{array}$ & $\begin{array}{l}\text { Mixed evergreen forest } \\
\text { dominated by scle- } \\
\text { rophyllous taxa that } \\
\text { require warm for bud- } \\
\text { burst. }\end{array}$ \\
\hline CGSS & $\begin{array}{l}\text { Cool grass- } \\
\text { lands }\end{array}$ & $\begin{array}{l}\text { Common above the } \\
\text { forest line of the Andes, } \\
\text { dominated by tussock } \\
\text { grasses and cushion } \\
\text { plants. }\end{array}$ & $\begin{array}{l}\text { Present only at the high- } \\
\text { est altitudes of the Andes }\end{array}$ & $\begin{array}{l}\text { Puna, Heath, Cushion } \\
\text { heath. }\end{array}$ & $\begin{array}{l}\text { Poaceae-dominated } \\
\text { cool grasslands with } \\
\text { occasional cushion } \\
\text { plants }\end{array}$ \\
\hline STEP & Steppe & $\begin{array}{l}\text { Dominated by grasses, } \\
\text { occasional shrubs and } \\
\text { steppe herbs. Profuse } \\
\text { flowering during the wet } \\
\text { season }\end{array}$ & $\begin{array}{l}\text { Extensive in eastern } \\
\text { Argentina, present in } \\
\text { lowland Central America } \\
\text { and northeast Brazil. }\end{array}$ & $\begin{array}{l}\text { Steppe grasslands, } \\
\text { Campo limpo, Pampa. }\end{array}$ & $\begin{array}{l}\text { Grasses and chenopods } \\
\text { forming low altitude } \\
\text { warm grasslands. }\end{array}$ \\
\hline
\end{tabular}

rain forest biome is present in three main areas: Amazonia, linear strips along the Atlantic coast and northeast South America extending into Central America. Forest associated with highland areas is divided into three biomes: warm evergreen forest, cool temperate rain forest and cool mixed forest (Fig. 4). Warm evergreen forest is most extensive along the lowland Andes, adjacent to the tropical rain forest. Cool mixed forest has a more restricted distribution, occupying a highland position until temperature becomes limiting for a number of taxa. Warm mixed forest is characterised by a mix of Pinus and Quercus species and is mainly restricted to Central America. The desert biome is restricted to coastal Peru, due to the Pacific Ocean anticyclone, this area receives very little moisture.

\section{Methods}

\subsection{Data sources}

Over the past five decades researchers have collected numerous pollen-based records from lakes and bogs (Table 3) 
Table 3. Characteristics of the sites from Latin America: specifically detailing location, potential vegetation around the sites, sample type, age range of sediments, number of $\mathrm{C}_{14}$ dates (RC), data type, principle analysts and associated references. Dating control (DC) codes are based on the COHMAP dating control scheme (Webb, 1985; Yu and Harrison, 1995). For sites with a continuous sedimentation (indicated by $\mathrm{C}$ after the numerical code), the dating control is based on bracketing dates as follows. 1: both dates within 2000 years of the selected interval, 2: one date within 2000 years the other within 4000 years, 3: both dates within 4000 years, 4: one date within 4000 one date within 6000 years, 5: both dates within 6000 years, 6: one date within 6000 the other within 8000 years, 7 bracketing dates more than 8000 years from the selected interval. For sites with discontinuous sedimentation (indicated by D after the numerical code), the dating control is based on single dates 1: indicated a date within 250 years of the selected interval, 2: a date within 500 years, 3: a date within 750 years, 4: a date within 1000 years, 5: a date within 1500 years, 6: a date within 2000 years, 7: a date of more than 2000 years from the selected interval.

\begin{tabular}{|c|c|c|c|c|c|c|c|c|c|c|c|c|c|}
\hline Site & Country & Long. & Latitude & Alt. & Age range & Present biome & $\begin{array}{c}\text { Sample } \\
\text { type }\end{array}$ & $\mathrm{RC}$ & $\begin{array}{l}\text { DC at } 6000 \\
\text { yr BP }\end{array}$ & $\begin{array}{c}\mathrm{DC} \text { at } 18000 \\
\text { yr BP }\end{array}$ & Data type & Analyst & Site publications \\
\hline Lake Åsa3 & Antarctica & -61.13 & -62.62 & 35 & $1280-4980$ & CGSH & Soil & 14 & - & - & Raw & Björck, S. & Björck et al. (1993) \\
\hline Harberton & Argentina & -67.16 & -54.88 & 20 & $0-13360$ & STEP & Mire & 16 & $1 \mathrm{C}$ & - & Raw & Markgraf, V. & $\begin{array}{l}\text { Markgraf }(1989,1991 \text {, } \\
1993)\end{array}$ \\
\hline Puerto del Hambre & Chile & -70.92 & -53.59 & 5 & $0-16000$ & CTRF & Mire & 5 & $4 \mathrm{C}$ & $7 \mathrm{D}$ & Digi & Heusser, C. & Heusser et al. (1995) \\
\hline La Misión & Argentina & -67.83 & -53.5 & 5 & $0-9990$ & STEP & Mire & 4 & $4 \mathrm{C}$ & - & Raw & Markgraf, V. & Markgraf (1993) \\
\hline Punta Arenas & Chile & -70.97 & -53.15 & 75 & $0-16500$ & CTRF & Mire & 5 & $4 \mathrm{C}$ & $7 \mathrm{D}$ & Digi & Heusser, C. & Heusser et al. (1995) \\
\hline Meseta Latorre 1 & Argentina & -72.05 & -51.52 & 980 & $240-7120$ & CGSH & Mire & 3 & $4 \mathrm{C}$ & - & Raw & Schäbitz, F & Schäbitz (1991) \\
\hline Meseta Latorre 2 & Argentina & -72.03 & -51.44 & 1000 & $0-7000$ & CGSH & Mire & 1 & $6 \mathrm{D}$ & - & Raw & Schäbitz, F. & Schäbitz (1991) \\
\hline Torres del Paine & Chile & -72.66 & -50.98 & 100 & $0-11000$ & CTRF & Lake & 8 & $2 \mathrm{C}$ & - & Digi & Heusser, C. & Heusser et al. (1995) \\
\hline Moreno Glacier Bog & Argentina & -73.00 & -50.46 & 200 & $0-9550$ & CTRF & Mire & 2 & $4 \mathrm{C}$ & - & Raw & Ager, T. A. & Mercer and Ager (1983) \\
\hline Patagonia & Argentina & -72.90 & -50.25 & 50 & Modern & CTRF & Soil & - & - & - & Digi & Mancini, M. V. & Mancini (1993) \\
\hline Patagonia & Argentina & -72.90 & -50.20 & 20 & Modern & CTRF & Soil & - & - & - & Digi & Mancini, M. V. & Mancini (1993) \\
\hline Patagonia & Argentina & -72.70 & -50.20 & 80 & Modern & CTRF & Soil & - & - & - & Digi & Mancini, M. V. & Mancini (1993) \\
\hline Patagonia & Argentina & -72.98 & -50.15 & 20 & Modern & CTRF & Soil & - & - & - & Digi & Mancini, M. V. & Mancini (1993) \\
\hline Patagonia & Argentina & -72.95 & -50.15 & 50 & Modern & CTRF & Soil & - & - & - & Digi & Mancini, M. V. & Mancini (1993) \\
\hline Patagonia & Argentina & -72.90 & -50.15 & 50 & Modern & CTRF & Soil & - & - & - & Digi & Mancini, M. V. & Mancini (1993) \\
\hline Patagonia & Argentina & -72.85 & -50.15 & 60 & Modern & CTRF & Soil & - & - & - & Digi & Mancini, M. V. & Mancini (1993) \\
\hline Patagonia & Argentina & -72.75 & -50.15 & 60 & Modern & CTRF & Soil & - & - & - & Digi & Mancini, M. V. & Mancini (1993) \\
\hline Patagonia & Argentina & -72.80 & -50.15 & 70 & Modern & CTRF & Soil & - & - & - & Digi & Mancini, M. V. & Mancini (1993) \\
\hline Patagonia & Argentina & -72.00 & -50.15 & 150 & Modern & CTRF & Soil & - & - & - & Digi & Mancini, M. V. & Mancini (1993) \\
\hline Patagonia & Argentina & -71.70 & -50.15 & 180 & Modern & CTRF & Soil & - & - & - & Digi & Mancini, M. V. & Mancini (1993) \\
\hline Patagonia & Argentina & -71.50 & -50.15 & 180 & Modern & CTRF & Soil & - & - & - & Digi & Mancini, M. V. & Mancini (1993) \\
\hline Patagonia & Argentina & -71.10 & -50.15 & 180 & Modern & CTRF & Soil & - & - & - & Digi & Mancini, M. V. & Mancini (1993) \\
\hline Patagonia & Argentina & -69.90 & -50.15 & 180 & Modern & CTRF & Soil & - & - & - & Digi & Mancini, M. V. & Mancini (1993) \\
\hline Patagonia & Argentina & -69.30 & -50.15 & 180 & Modern & CTRF & Soil & - & - & - & Digi & Mancini, M. V. & Mancini (1993) \\
\hline Patagonia & Argentina & -71.30 & -50.15 & 190 & Modern & CTRF & Soil & - & - & - & Digi & Mancini, M. V. & Mancini (1993) \\
\hline Patagonia & Argentina & -70.90 & -50.15 & 190 & Modern & CTRF & Soil & - & - & - & Digi & Mancini, M. V. & Mancini (1993) \\
\hline Patagonia & Argentina & -70.20 & -50.15 & 190 & Modern & CTRF & Soil & - & - & - & Digi & Mancini, M. V. & Mancini (1993) \\
\hline Patagonia & Argentina & -69.60 & -50.15 & 190 & Modern & CTRF & Soil & - & - & - & Digi & Mancini, M. V. & Mancini (1993) \\
\hline Patagonia & Argentina & -70.50 & -50.15 & 200 & Modern & CTRF & Soil & - & - & - & Digi & Mancini, M. V. & Mancini (1993) \\
\hline Patagonia & Argentina & -72.55 & -50.10 & 100 & Modern & CTRF & Soil & - & - & - & Digi & Mancini, M. V. & Mancini (1993) \\
\hline Patagonia & Argentina & -71.95 & -50.10 & 180 & Modern & CTRF & Soil & - & - & - & Digi & Mancini, M. V. & Mancini (1993) \\
\hline Patagonia & Argentina & -69.00 & -50.10 & 190 & Modern & CTRF & Soil & - & - & - & Digi & Mancini, M. V. & Mancini (1993) \\
\hline Patagonia & Argentina & -72.90 & -50.05 & 50 & Modern & CTRF & Soil & - & - & - & Digi & Mancini, M. V. & Mancini (1993) \\
\hline Patagonia & Argentina & -68.60 & -50.05 & 50 & Modern & CTRF & Soil & - & - & - & Digi & Mancini, M. V. & Mancini (1993) \\
\hline Patagonia & Argentina & -68.90 & -50.05 & 100 & Modern & CTRF & Soil & - & - & - & Digi & Mancini, M. V. & Mancini (1993) \\
\hline Patagonia & Argentina & -72.00 & -50.05 & 150 & Modern & CTRF & Soil & - & - & - & Digi & Mancini, M. V. & Mancini (1993) \\
\hline Patagonia & Argentina & -68.10 & -50.00 & 0 & Modern & CTRF & Soil & - & - & - & Digi & Mancini, M. V. & Mancini (1993) \\
\hline Patagonia & Argentina & -68.30 & -50.00 & 20 & Modern & CTRF & Soil & - & - & - & Digi & Mancini, M. V. & Mancini (1993) \\
\hline Dichan & Chile & -73.88 & -49.66 & 50 & $0-8000$ & CTRF & Mire & 5 & $2 \mathrm{C}$ & - & Digi & Heusser, C. & Heusser et al. (1995) \\
\hline Puerto Eden & Chile & -74.41 & -49.13 & 50 & $0-14750$ & CTRF & Mire & 7 & $4 \mathrm{C}$ & - & Raw & Markgraf, V. & $\begin{array}{l}\text { Ashworth and Markgraf } \\
\text { (1989); Ashworth et al. } \\
\text { (1991) }\end{array}$ \\
\hline La Esperanza & Chile & -72.83 & -46.63 & 330 & $0-1500$ & CTRF & Mire & - & - & - & Raw & Graf, $\mathrm{K}$. & Graf (1992) \\
\hline LagoBsAs & Argentina & -71.45 & -46.44 & 230 & Modern & CTRF & Soil & - & - & - & Raw & Schäbitz, F. & Schäbitz (1994) \\
\hline Laguna Stibnite & Chile & -74.43 & -46.43 & 50 & $0-14000$ & CTRF & Lake & 6 & $1 \mathrm{C}$ & $4 \mathrm{D}$ & Digi & Lumley, S. & $\begin{array}{l}\text { Lumley and Switsur } \\
\text { (1993) }\end{array}$ \\
\hline Laguna Stibnite & Chile & -74.38 & -46.43 & 50 & $0-17500$ & CTRF & Lake & 10 & $1 \mathrm{C}$ & $2 \mathrm{D}$ & Digi & Bennet, $\mathrm{K}$. & Bennet et al. (2000) \\
\hline Laguna Six Minutes & Chile & -74.33 & -46.43 & 50 & $0-17500$ & CTRF & Lake & 4 & $4 \mathrm{C}$ & 7D & Digi & Bennet, $\mathrm{K}$. & Bennet et al. (2000) \\
\hline Pico Salam & Argentina & -67.43 & -45.42 & 637 & Modern & STEP & Soil & - & - & - & Raw & Schäbitz, F. & Schäbitz (1994) \\
\hline Laguna Lincoln & Chile & -74.07 & -45.34 & 50 & $0-16500$ & CTRF & Lake & 5 & $2 \mathrm{C}$ & $7 \mathrm{D}$ & Digi & Bennet, $\mathrm{K}$. & Bennet et al. (2000) \\
\hline Laguna Lofel & Chile & -74.43 & -44.85 & 50 & $0-16500$ & CTRF & Lake & 5 & $2 \mathrm{C}$ & $6 \mathrm{D}$ & Digi & Bennet, $\mathrm{K}$. & Bennet et al. (2000) \\
\hline Estero Huitanque & Chile & -73.82 & -43.61 & 52 & $0-14000$ & CTRF & Mire & 9 & $1 \mathrm{C}$ & - & Digi & Heusser, C. & Heusser et al. (1995) \\
\hline AustroEsqu & Argentina & -71.47 & -42.66 & 1100 & Modern & CTRF & Soil & - & - & - & Raw & Schäbitz, F. & Schäbitz (1994) \\
\hline Mayol & Chile & -73.75 & -42.64 & 75 & $0-14000$ & CTRF & Mire & 12 & $3 \mathrm{C}$ & - & Digi & Heusser, C. & Heusser et al. (1995) \\
\hline Puchilco & Chile & -73.62 & -42.63 & 110 & $0-12500$ & CTRF & Mire & 7 & $2 \mathrm{C}$ & - & Digi & Heusser, C. & Heusser et al. (1995) \\
\hline AlercesNor & Argentina & -71.60 & -42.56 & 800 & Modern & CTRF & Soil & - & - & - & Raw & Schäbitz, F. & Schäbitz (1994) \\
\hline San Pedro & Chile & -73.95 & -42.25 & 650 & Modern & CTRF & Mire & - & - & - & Raw & Moar, N. T. & Godley and Moar (1973) \\
\hline Chepu & Chile & -73.66 & -42.17 & 140 & Modern & CTRF & Mire & & - & - & Raw & Moar, N. T. & Godley and Moar (1973) \\
\hline Mallin Book & Argentina & -71.58 & -41.33 & 800 & $290-14200$ & CTRF & Mire & 9 & $1 \mathrm{C}$ & - & Raw & Markgraf, V. & Markgraf (1983) \\
\hline Comallo & Argentina & -70.21 & -41.01 & 815 & Modern & CGSH & Soil & - & - & - & Raw & Schäbitz, F. & Schäbitz (1994) \\
\hline Puerto Octay PM13 & Chile & -72.90 & -40.93 & 120 & $500-20000$ & CTRF & Mire & 16 & $4 \mathrm{C}$ & $1 \mathrm{C}$ & Raw & Moreno, P. I. & Moreno (1994) \\
\hline Espuma & Argentina & -63.25 & -40.67 & 50 & Modern & TDFO & Soil & - & - & - & Raw & Schäbitz, F. & Schäbitz (1994) \\
\hline Primavera & Argentina & -71.18 & -40.66 & 800 & $1800-4380$ & CTRF & Midden & 6 & - & - & Raw & Markgraf, V. & Markgraf et al. (2009) \\
\hline Encantado & Argentina & -71.13 & -40.66 & 960 & $290-1260$ & CTRF & Midden & 3 & - & - & Raw & Markgraf, V. & Markgraf et al. (2009) \\
\hline Ruta 3.4 & Argentina & -62.79 & -40.50 & 20 & Modern & STEP & Soil & - & - & - & Raw & Schäbitz, F. & Schäbitz (1994) \\
\hline Ruta 3.3 & Argentina & -62.59 & -40.08 & 20 & Modern & STEP & Soil & - & - & - & Raw & Schäbitz, F. & Schäbitz (1994) \\
\hline Caunahue & Chile & -72.00 & -40.00 & 500 & $4370-14930$ & CTRF & Section & 9 & $2 \mathrm{C}$ & - & Raw & Markgraf, V. & Markgraf (1991) \\
\hline Ruta 250.19 & Argentina & -65.58 & -39.54 & 117 & Modern & STEP & Soil & - & - & - & Raw & Schäbitz, F. & Schäbitz (1994) \\
\hline
\end{tabular}


Table 3. Continued.

\begin{tabular}{|c|c|c|c|c|c|c|c|c|c|c|c|c|c|}
\hline Site & Country & Long. & Latitude & Alt. & Age range & Present biome & $\begin{array}{l}\text { Sample } \\
\text { type }\end{array}$ & $\mathrm{RC}$ & $\begin{array}{c}\mathrm{DC} \text { at } 6000 \\
\text { yr BP }\end{array}$ & $\begin{array}{c}\mathrm{DC} \text { at } 18000 \\
\text { yr BP }\end{array}$ & Data type & Analyst & Site publications \\
\hline Pedro Luro & Argentina & -62.53 & -39.50 & 20 & Modern & STEP & Soil & - & - & - & Raw & Schäbitz, F. & Schäbitz (1994) \\
\hline Origone & Argentina & -62.43 & -39.08 & 20 & Modern & STEP & Soil & - & - & - & Raw & Schäbitz, F. & Schäbitz (1994) \\
\hline Gaviotas & Argentina & -63.65 & -39.07 & 90 & Modern & TDFO & Soil & - & - & - & Raw & Schäbitz, F. & Schäbitz (1994) \\
\hline Salina Anzotegui & Argentina & -63.77 & -39.06 & -5 & $0-10360$ & STEP & Playa & 5 & 3D & - & Raw & Schäbitz, F. & Schäbitz (1994) \\
\hline Cueva Haichol & Argentina & -70.66 & -38.58 & 1050 & $200-6890$ & STEP & Cave & 2 & $1 \mathrm{C}$ & - & Raw & Markgraf, v. & Markgraf (1988) \\
\hline Arroyo Sauce Chico & Argentina & -62.23 & -38.07 & 85 & Modern & STEP & Soil & - & - & - & Raw & Prieto, A. R. & Prieto (1996) \\
\hline Cerro La China & Argentina & -58.64 & -37.84 & 200 & $0-10500$ & STEP & Soil & 2 & $4 \mathrm{C}$ & - & Raw & Prieto, A. R. & $\begin{array}{l}\text { Prieto and Páez } \\
\text { (1989); Páez and } \\
\text { Prieto (1993) }\end{array}$ \\
\hline Empalme Querandíes & Argentina & -60.65 & -37.00 & 105 & $0-15000$ & STEP & Lake & 8 & $2 \mathrm{C}$ & - & Raw & Prieto, A. R. & Prieto (1996) \\
\hline Veranada Pelan & Argentina & -70.38 & -36.88 & 1860 & $0-10890$ & CGSH & Mire & 3 & $3 \mathrm{C}$ & - & Raw & Schäbitz, F. & Schäbitz (1989) \\
\hline Vaca Lauquen & Argentina & -71.08 & -36.83 & 1450 & $0-11260$ & CTRF & Mire & 3 & $1 \mathrm{C}$ & - & Raw & Markgraf, V. & Markgraf (1987) \\
\hline Veranada Vulkanpickel & Argentina & -70.41 & -36.68 & 2800 & $0-7790$ & CGSH & Mire & 1 & 7D & - & Raw & Schäbitz, F. & Schäbitz (1989) \\
\hline Salado & Argentina & -69.75 & -35.33 & 3200 & $20-4330$ & CGSH & Mire & 2 & - & - & Raw & Markgraf, V. & Markgraf (1983) \\
\hline Salina 2 & Argentina & -69.33 & -32.25 & 2000 & $100-6510$ & CGSH & Mire & 2 & $1 \mathrm{C}$ & - & Raw & Markgraf, v. & Markgraf (1983) \\
\hline Serra do Rio Rastro & Brazil & -49.55 & -28.55 & 1420 & $800-11180$ & WTRF & Mire & 3 & $2 \mathrm{C}$ & - & Raw & Behling, $\mathrm{H}$. & $\begin{array}{l}\text { Behling (1993); } \\
\text { Behling (1997a, b) }\end{array}$ \\
\hline Morro da Igreja & Brazil & -49.86 & -28.18 & 1800 & $0-10390$ & WTRF & Mire & 2 & - & - & Raw & Behling, $\mathrm{H}$. & $\begin{array}{l}\text { Behling (1993); } \\
\text { Behling (1997a, b) }\end{array}$ \\
\hline Serra da Boa Vista & Brazil & -49.15 & -27.70 & 1160 & $0-14000$ & WTRF & Mire & 4 & $2 \mathrm{C}$ & - & Raw & Behling, $\mathrm{H}$. & $\begin{array}{l}\text { Behling (1993); } \\
\text { Behling (1997a, b) }\end{array}$ \\
\hline Rano Kao & Chile & -109.43 & -27.18 & 110 & $0-1360$ & TDFO & Lake & 2 & - & - & Raw & Flenley, J. & $\begin{array}{l}\text { Flenley and King } \\
\text { (1984); Flenley et } \\
\text { al. (1991) }\end{array}$ \\
\hline Rano Raraku Bore 3 & Chile & -109.28 & -27.16 & 75 & $0-35260$ & TDFO & Lake & 10 & $4 \mathrm{C}$ & $4 \mathrm{C}$ & Raw & Flenley, J. & $\begin{array}{l}\text { Flenley and King } \\
\text { (1984); Flenley et } \\
\text { al. (1991) }\end{array}$ \\
\hline Rano Aroui & Chile & -109.40 & -27.08 & 425 & $0-37600$ & TDFO & Lake & 11 & $2 \mathrm{C}$ & $6 \mathrm{C}$ & Raw & Flenley, J. & $\begin{array}{l}\text { Flenley and King } \\
\text { (1984) }\end{array}$ \\
\hline Poço Grande & Brazil & -48.86 & -26.41 & 10 & $0-4840$ & WTRF & Section & 4 & - & - & Raw & Behling, $\mathrm{H}$. & $\begin{array}{l}\text { Behling (1993); } \\
\text { Behling (1997a, b) }\end{array}$ \\
\hline Reserva Volta Velha & Brazil & -48.38 & -26.04 & 0 & Modern & WTRF & Trap & - & - & - & Digi & Behling, $\mathrm{H}$. & $\begin{array}{l}\text { Behling et al. } \\
\text { (1997) }\end{array}$ \\
\hline Atlantic & Brazil & -48.35 & -25.95 & 200 & Modern & WTRF & Trap & - & - & - & Raw & Behling, $\mathrm{H}$. & $\begin{array}{l}\text { Behling et al. } \\
\text { (1997) }\end{array}$ \\
\hline Colombo & Brazil & -49.23 & -25.33 & 920 & Modern & TSFO & Trap & - & - & - & Raw & Behling, $\mathrm{H}$. & $\begin{array}{l}\text { Behling et al. } \\
\text { (1997) }\end{array}$ \\
\hline Serra Campos Gerais & Brazil & -50.21 & -24.66 & 1200 & $0-12480$ & WTRF & Mire & 4 & $3 \mathrm{C}$ & - & Raw & Behling, $\mathrm{H}$. & Behling (1997a) \\
\hline Aguilar & Argentina & -65.75 & -23.83 & 4000 & $0-9830$ & CGSH & Mire & 3 & $2 \mathrm{C}$ & - & Raw & Markgraf, v. & Markgraf (1985) \\
\hline Rio da Curuá & Brazil & -48.83 & -23.83 & 800 & $0-7500$ & WTRF & Lake & 4 & - & - & Raw & Behling, $\mathrm{H}$. & $\begin{array}{l}\text { Behling et al. } \\
\text { (1997) }\end{array}$ \\
\hline Tumbre 2 & Chile & -67.78 & -23.31 & 3880 & $241-7500$ & CGSH & Lake & 3 & $2 \mathrm{C}$ & - & Raw & Graf, $\mathrm{K}$. & Graf (1992) \\
\hline Aguas Calientas & Chile & -67.42 & -23.08 & 4210 & $0-6400$ & CGSH & Mire & 1 & 7D & - & Raw & Graf, K. & Graf (1992) \\
\hline Botucatu & Brazil & -48.00 & -23.00 & 700 & Modern & WTRF & Soil & - & - & - & Raw & Behling, $\mathrm{H}$. & Behling (2009) \\
\hline Curcuab & Brazil & -48.00 & -23.00 & 700 & Modern & WTRF & Soil & - & - & - & Raw & Behling, $\mathrm{H}$. & Behling (2009) \\
\hline Lagoa da Caço & Brazil & -43.43 & -22.97 & 5 & $3000-20000$ & TDFO & Lake & 14 & $1 \mathrm{C}$ & - & Raw & Behling, $\mathrm{H}$. & $\begin{array}{l}\text { Ledru et al. } \\
(2001)\end{array}$ \\
\hline Morro de Itapeva & Brazil & -45.63 & -22.78 & 1850 & $0-35010$ & WTRF & Lake & 9 & $4 \mathrm{C}$ & - & Raw & Behling, $\mathrm{H}$. & Behling (1997b) \\
\hline Assis & Brazil & -50.50 & -22.68 & 540 & Modern & TSFO & Soil & - & - & - & Raw & Behling, $\mathrm{H}$. & Behling (2009) \\
\hline Lagoa Santa & Brazil & -47.45 & -22.36 & 630 & Modern & WTRF & River & - & - & - & Digi & Parizzi, M. G. & $\begin{array}{l}\text { Salgado-Labouriau } \\
\text { et al. (1998) }\end{array}$ \\
\hline Bauru & Brazil & -49.07 & -22.32 & 570 & Modern & TSFO & Soil & - & - & - & Raw & Behling, $\mathrm{H}$. & Behling (2009) \\
\hline Brotas & Brazil & -48.08 & -22.29 & 700 & Modern & WTRF & Soil & - & - & - & Raw & Behling, H. & Behling (2009) \\
\hline Salitre & Brazil & -46.78 & -19.00 & 1050 & $0-50000$ & WTRF & Lake & 14 & $1 \mathrm{C}$ & $7 \mathrm{C}$ & Raw & Ledru, M.-P. & $\begin{array}{l}\text { Ledru (1992, } \\
\text { 1993); Ledru et al. } \\
(1994,1996)\end{array}$ \\
\hline Ajata & Chile & -69.20 & -18.25 & 4700 & $0-1460$ & CGSH & Mire & 1 & - & - & Raw & Graf, K. & Graf (1992) \\
\hline Sajama & Bolivia & -68.88 & -18.16 & 4250 & $0-4400$ & CGSH & Lake & 9 & - & - & Raw & Graf, K. & Graf (1992) \\
\hline Lago do Pires & Brazil & -42.21 & -17.95 & 390 & $0-9720$ & TSFO & Lake & 7 & $1 \mathrm{C}$ & - & Raw & Behling, $\mathrm{H}$. & $\begin{array}{l}\text { Behling (1993); } \\
\text { Behling (1997a, b) }\end{array}$ \\
\hline Wasa Mayu & Bolivia & -65.91 & -17.54 & 2720 & $1000-31000$ & COMI & Lake & 1 & 7D & 7D & Raw & Graf, K. & Graf (1992) \\
\hline Crominia & Brazil & -49.45 & -17.28 & 200 & $0-32200$ & TDFO & Palm swamp & 5 & $2 \mathrm{C}$ & $1 \mathrm{D}$ & Digi & $\begin{array}{l}\text { Salgado- } \\
\text { Labouriau, M. L. }\end{array}$ & $\begin{array}{l}\text { Salgado-Labouriau } \\
\text { et al. (1998) }\end{array}$ \\
\hline Mt. Blanco & Bolivia & -67.35 & -17.02 & 4780 & $1250-7500$ & CGSH & Lake & 7 & $1 \mathrm{C}$ & - & Raw & Graf, K. & Graf (1992) \\
\hline Lake Huinãmimarca & Bolivia & -69.00 & -16.50 & 3765 & $0-25000$ & CSGH & Lake & 48 & $1 \mathrm{C}$ & $3 \mathrm{C}$ & Digi & Mourguiart, P. H. & $\begin{array}{l}\text { Mourguiart et al. } \\
\text { (1995); Argollo } \\
\text { and Mourguiart } \\
(2000)\end{array}$ \\
\hline Cerro Calvario & Bolivia & -68.50 & -16.50 & 3950 & $0-8360$ & CGSH & Mire & 4 & $1 \mathrm{C}$ & - & Raw & Graf, K. & Graf (1992) \\
\hline Rio Kaluyo & Bolivia & -68.13 & -16.43 & 4070 & $130-9920$ & CGSH & Lake & 3 & $3 \mathrm{C}$ & - & Raw & Graf, $\mathrm{K}$. & Graf (1992) \\
\hline Chacaltaya 1 & Bolivia & -68.13 & -16.36 & 4750 & $80-7400$ & CGSH & Mire & 1 & $1 \mathrm{C}$ & - & Raw & Graf, K. & Graf (1992) \\
\hline Cumre Unduavi & Bolivia & -68.03 & -16.33 & 4620 & $0-9200$ & CGSH & Mire & 6 & $3 \mathrm{C}$ & - & Raw & Graf, K. & Graf (1992) \\
\hline Rio Jequitinhonha & Brazil & -38.92 & -15.85 & 80 & Modern & TDFO & River & - & - & - & Raw & Behling, $\mathrm{H}$. & $\begin{array}{l}\text { Behling et al. } \\
(2000)\end{array}$ \\
\hline Brasilia 1 & Brazil & -47.66 & -15.59 & 1030 & Modern & TDFO & Soil & - & - & - & Raw & Behling, $\mathrm{H}$. & Behling (2009) \\
\hline Aguads Emendadas & Brazil & -47.58 & -15.56 & 200 & $0-28000$ & TDFO & Palm swamp & 6 & $7 \mathrm{C}$ & $7 \mathrm{D}$ & Digi & $\begin{array}{l}\text { Salgado- } \\
\text { Labouriau, M. L. }\end{array}$ & $\begin{array}{l}\text { Salgado-Labouriau } \\
\text { et al. (1998) }\end{array}$ \\
\hline Cuiaba & Brazil & -55.86 & -15.35 & 350 & Modern & TDFO & Soil & - & - & - & Raw & Behling, $\mathrm{H}$. & Behling (2009) \\
\hline Amarete & Bolivia & -68.98 & -15.23 & 4000 & $0-9160$ & CGSH & Mire & 2 & $5 \mathrm{D}$ & - & Raw & Graf, K. & Graf (1992) \\
\hline Cotapampa & Bolivia & -69.11 & -15.21 & 4450 & $0-9560$ & CGSH & Mire & 5 & $2 \mathrm{C}$ & - & Raw & Graf, $\mathrm{K}$. & Graf (1992) \\
\hline Katantica & Bolivia & -69.18 & -14.8 & 4820 & $50-7720$ & CGSH & Mire & 3 & $1 \mathrm{C}$ & - & Raw & Graf, K. & Graf (1992) \\
\hline Laguna Chaplin & Bolivia & -61.05 & -14.50 & 750 & $0-40000$ & TSFO & Lake & 14 & $2 \mathrm{C}$ & $1 \mathrm{D}$ & Digi & Mayle, F. & $\begin{array}{l}\text { Mayle et al. } \\
(2000)\end{array}$ \\
\hline Rio de Contas & Brazil & -39.00 & -14.28 & 80 & Modern & TDFO & River & - & - & - & Raw & Behling, $\mathrm{H}$. & $\begin{array}{l}\text { Behling et al. } \\
(2000)\end{array}$ \\
\hline Laguna Bella Vista & Bolivia & -61.56 & -13.58 & 750 & $0-55000$ & TSFO & Lake & 15 & $2 \mathrm{C}$ & $1 \mathrm{D}$ & Digi & Mayle, F. & $\begin{array}{l}\text { Mayle et al. } \\
(2000)\end{array}$ \\
\hline Laguna Jeronimo & Peru & -75.21 & -11.78 & 4450 & $0-11260$ & CGSH & Lake & 4 & $4 \mathrm{C}$ & - & Raw & Hansen, B. C. S. & $\begin{array}{l}\text { Hansen et al. } \\
\text { (1994) }\end{array}$ \\
\hline
\end{tabular}


Table 3. Continued.

\begin{tabular}{|c|c|c|c|c|c|c|c|c|c|c|c|c|c|}
\hline Site & Country & Long. & Latitude & Alt. & Age range & Present biome & $\begin{array}{c}\text { Sample } \\
\text { type }\end{array}$ & $\mathrm{RC}$ & $\begin{array}{c}\text { DC at } 6000 \\
\text { yr BP }\end{array}$ & $\begin{array}{c}\mathrm{DC} \text { at } 18000 \\
\mathrm{yr} \mathrm{BP}\end{array}$ & Data type & Analyst & Site publications \\
\hline Laguna Pomacocha & Peru & -75.50 & -11.75 & 4450 & $4100-10220$ & CGSH & Lake & 4 & $1 \mathrm{C}$ & - & Raw & Hansen, B. C. S. & $\begin{array}{l}\text { Hansen et al. } \\
\text { (1994) }\end{array}$ \\
\hline Laguna Paca & Peru & -75.50 & -11.71 & 3600 & $0-5410$ & CGSH & Lake & 1 & 4D & & Raw & Hansen, B. C. S. & $\begin{array}{l}\text { Hansen and Rod- } \\
\text { bell (1995) }\end{array}$ \\
\hline Laguna Tuctua & Peru & -75.00 & -11.66 & 4250 & $0-11390$ & CGSH & Lake & 4 & $2 \mathrm{C}$ & - & Raw & Hansen, B. C. S. & $\begin{array}{l}\text { Hansen et al. } \\
\text { (1994) }\end{array}$ \\
\hline Laguna Milloc & Peru & -76.35 & -11.56 & 4325 & $280-11050$ & CGSH & Lake & 1 & $3 \mathrm{C}$ & - & Raw & Graf, K. & Graf (1992) \\
\hline Laguna Junin2 & Peru & -76.18 & -11.00 & 4100 & $0-43000$ & CGSH & Lake & 11 & $6 \mathrm{C}$ & $1 \mathrm{C}$ & Raw & Hansen, B. C. S. & $\begin{array}{l}\text { Hansen and Rod- } \\
\text { bell (1995) }\end{array}$ \\
\hline Laguna Huatacocha & Peru & -76.55 & -10.76 & 4500 & $0-10620$ & CGSH & Lake & 5 & $2 \mathrm{C}$ & - & Raw & Hansen, B. C. S. & $\begin{array}{l}\text { Hansen and Rod- } \\
\text { bell (1995) }\end{array}$ \\
\hline Rio São Francisco & Brazil & -43.00 & -10.46 & 400 & $0-11500$ & TDFO & River & 6 & $1 \mathrm{C}$ & - & Digi & De Oliveira, P. E. & $\begin{array}{l}\text { De Oliveira et al. } \\
\text { (1999) }\end{array}$ \\
\hline Saquinho & Brazil & -43.23 & -10.44 & 480 & $0-11000$ & TSFO & Mire & 6 & $1 \mathrm{C}$ & - & Digi & De Oliveira, P. E. & $\begin{array}{l}\text { De Oliveira et al. } \\
\text { (1999) }\end{array}$ \\
\hline Rio São Francisco & Brazil & -36.50 & -10.26 & 80 & Modern & TDFO & River & - & - & - & Raw & Behling, $\mathrm{H}$. & $\begin{array}{l}\text { Behling et al. } \\
(2000)\end{array}$ \\
\hline Katira & Brazil & -63.00 & -9.00 & 750 & $0-60000$ & TDFO & Lake & 4 & $7 \mathrm{D}$ & $1 \mathrm{D}$ & Digi & $\begin{array}{l}\text { van der Hammen, } \\
\text { T. }\end{array}$ & $\begin{array}{l}\text { van der Hammen } \\
\text { and Absy (1994) }\end{array}$ \\
\hline Campina Grande I & Brazil & -35.75 & -7.23 & 70 & Modern & TSFO & Soil & - & - & - & Raw & Behling, H. & $\begin{array}{l}\text { Behling et al. } \\
(2000)\end{array}$ \\
\hline Lagoa Grande & Brazil & -47.45 & -7.08 & 75 & Modern & TDFO & Lake & - & - & - & Raw & Behling, $\mathrm{H}$. & $\begin{array}{l}\text { Behling et al. } \\
\text { (2000) }\end{array}$ \\
\hline Picos & Brazil & -41.40 & -7.06 & 70 & Modern & TDFO & Soil & - & $1 \mathrm{D}$ & $1 \mathrm{D}$ & Raw & Harbele, S. & $\begin{array}{l}\text { Behling et al. } \\
(2000)\end{array}$ \\
\hline Lago Bolim & Brazil & -35.18 & -6.04 & 90 & Moden & TDFO & Lake & - & - & - & Raw & Behling, $\mathrm{H}$. & $\begin{array}{l}\text { Behling et al. } \\
(2000)\end{array}$ \\
\hline Rio Protengi & Brazil & -35.25 & -5.78 & 80 & Modern & TDFO & River & - & - & - & Raw & Behling, $\mathrm{H}$. & $\begin{array}{l}\text { Behling et al. } \\
(2000)\end{array}$ \\
\hline Mirim & Brazil & -35.30 & -5.68 & 70 & Modern & TDFO & Soil & - & $1 \mathrm{D}$ & $7 \mathrm{D}$ & Raw & Ledru, M.-P. & $\begin{array}{l}\text { Behling et al. } \\
(2000)\end{array}$ \\
\hline Rio Mirim & Brazil & -35.40 & -5.64 & 70 & Modern & TDFO & River & - & - & - & Raw & Behling, $\mathrm{H}$. & $\begin{array}{l}\text { Behling et al. } \\
\text { (2000) }\end{array}$ \\
\hline Rio (unclear) & Brazil & -38.00 & -5.50 & 50 & Modern & TDFO & River & - & - & - & Raw & Behling, $\mathrm{H}$. & $\begin{array}{l}\text { Behling et al. } \\
\text { (2000) }\end{array}$ \\
\hline Carajas & Brazil & -48.00 & -5.00 & 150 & $0-20000$ & TSFO & Lake & 8 & $2 \mathrm{C}$ & $6 \mathrm{C}$ & Digi & Absy, M. L. & $\begin{array}{l}\text { Absy et al. } \\
\text { (1991) }\end{array}$ \\
\hline Ciudad Universitaria $X$ & Colombia & -74.18 & -4.75 & 2560 & $0->35000$ & COMI & Lake & 4 & $4 \mathrm{C}$ & $7 \mathrm{D}$ & Raw & $\begin{array}{l}\text { van der Hammen, } \\
\text { T. }\end{array}$ & $\begin{array}{ll}\text { van der Hammen } \\
\text { and } & \text { González } \\
(1960) & \end{array}$ \\
\hline Rio Jaguaribe II & Brazil & -37.76 & -4.55 & 50 & Modern & TDFO & River & - & - & - & Raw & Behling, $\mathrm{H}$. & $\begin{array}{l}\text { Behling et al. } \\
(2000)\end{array}$ \\
\hline Rio Jaguaribe I & Brazil & -37.75 & -4.43 & 50 & Modern & TDFO & River & - & - & - & Raw & Behling, $\mathrm{H}$. & $\begin{array}{l}\text { Behling et al. } \\
(2000)\end{array}$ \\
\hline Lake Surucucho & Ecuador & -78.95 & -3.75 & 970 & $0-12000$ & WTRF & Lake & 9 & $4 \mathrm{C}$ & - & Digi & Colinvaux, P. & $\begin{array}{l}\text { Colinvaux et al. } \\
\text { (1997) }\end{array}$ \\
\hline Ayauch & Ecuador & -78.13 & -2.09 & 550 & $0-7500$ & WTRF & Lake & 4 & $2 \mathrm{C}$ & - & Digi & Bush, M. & $\begin{array}{l}\text { Bush et al. (1990); } \\
\text { Bush and Colin- } \\
\text { vaux (1988); Col- } \\
\text { invaux et al. (1988) }\end{array}$ \\
\hline Llaviucu & Ecuador & -79.43 & -1.83 & 3120 & Modern & CTRF & Lake & - & - & - & Digi & Bush, M. & Bush et al. (1990) \\
\hline Kumpack & Ecuador & -78.51 & -1.53 & 700 & Modern & WTRF & Lake & - & - & - & Digi & Bush, M. & Bush et al. (1990) \\
\hline Indanza & Ecuador & -78.83 & -1.53 & 2100 & Modern & CTRF & Lake & - & - & - & Digi & Bush, M. & Bush et al. (1990) \\
\hline Comprida & Brazil & -53.15 & -1.5 & 130 & $0-7200$ & TRFO & Lake & 5 & $1 \mathrm{C}$ & - & Digi & Bush, M. & Bush et al. (2000) \\
\hline Geral & Brazil & -53.00 & -1.5 & 130 & $0-6000$ & TRFO & Lake & 2 & $1 \mathrm{C}$ & - & Digi & Bush, M. & Bush et al. (2000) \\
\hline Puyo Bog & Ecuador & -79.06 & -1.43 & 953 & Modern & WTFO & Lake & - & - & - & Digi & Bush, M. & Bush et al. (1990) \\
\hline Rum Tum & Ecuador & -79.03 & -1.13 & 2392 & Modern & CTRF & Lake & - & - & - & Digi & Bush, M. & Bush et al. (1990) \\
\hline Yambo & Ecuador & -79.03 & -1.03 & 2600 & Modern & CTRF & Lake & - & - & - & Digi & Bush, M. & Bush et al. (1990) \\
\hline Lago Crispim & Brazil & -48.00 & -0.8 & 0 & $0-9000$ & TRFO & Lake & 4 & $1 \mathrm{C}$ & - & Raw & Behling, $\mathrm{H}$. & $\begin{array}{l}\text { Behling et al. } \\
\text { (1997) }\end{array}$ \\
\hline Lagoa da Curuça2 & Brazil & -47.85 & -0.76 & 35 & $0-9440$ & TRFO & Lake & 4 & $2 \mathrm{C}$ & - & Raw & Behling, $\mathrm{H}$. & Behling (1996) \\
\hline Mariñame-II & Colombia & -72.03 & -0.66 & 160 & $0-5000$ & TRFO & Lake & 5 & $1 \mathrm{C}$ & - & Raw & Behling, $\mathrm{H}$. & $\begin{array}{l}\text { Behling et al. } \\
\text { (1999) }\end{array}$ \\
\hline Monica-1 & Colombia & -72.50 & -0.60 & 160 & $0-12000$ & TRFO & Lake & 3 & $2 \mathrm{C}$ & - & Raw & Behling, $\mathrm{H}$. & $\begin{array}{l}\text { Behling et al. } \\
\text { (1999) }\end{array}$ \\
\hline Añangucocha & Ecuador & -77.03 & -0.53 & 280 & Modern & TRFO & Lake & - & - & - & Digi & Bush, M. & Bush et al. (1990) \\
\hline Limoncocha & Ecuador & -76.66 & -0.38 & 230 & $0-1300$ & TRFO & Lake & 2 & - & - & Digi & Colinvaux, $\mathrm{P}$. & $\begin{array}{l}\text { Colinvaux et al. } \\
\text { (1988) }\end{array}$ \\
\hline Cayambe & Ecuador & -78.03 & -0.03 & 4350 & $0-5200$ & CGSH & Mire & 6 & $4 \mathrm{D}$ & - & Raw & Graf, K. & Graf $(1989,1992)$ \\
\hline Cuyabeno & Ecuador & -77.01 & 0 & 280 & Modern & TRFO & Lake & - & - & - & Digi & Bush, M. & Bush et al. (1990) \\
\hline Lago Agrio & Ecuador & -77.03 & 0.03 & 330 & Modern & TRFO & Lake & - & - & - & Digi & Bush, M. & $\begin{array}{l}\text { Bush et al. (1990); } \\
\text { Colinvaux et al. } \\
\text { (1988) }\end{array}$ \\
\hline San Marcos & Ecuador & -79.03 & 0.03 & 3400 & Modern & CGSH & Mire & - & - & - & Digi & Bush, M. & Bush et al. (1990) \\
\hline Santa Cecilia & Ecuador & -77.03 & 0.04 & 330 & Modern & TRFO & Lake & - & - & - & Digi & Bush, M. & Bush et al. (1990) \\
\hline Lake Santa Cecilia & Ecuador & -77.02 & 0.06 & 330 & $0-1000$ & TRFO & Lake & 2 & - & - & Digi & Colinvaux, P. & $\begin{array}{l}\text { Colinvaux et al. } \\
\text { (1988); Bush et al. } \\
\text { (1990) }\end{array}$ \\
\hline Cunro & Ecuador & -79.03 & 0.08 & 2800 & Modern & CTRF & Lake & - & - & - & Digi & Bush, M. & Bush et al. (1990) \\
\hline Mera & Ecuador & -76.92 & 0.11 & 1100 & Modern & WTRF & Lake & - & - & - & Digi & Bush, M. & Bush et al. (1990) \\
\hline Yaguara cocha & Ecuador & -79.03 & 0.13 & 2210 & Modern & CTRF & Lake & - & - & - & Raw & Bush, M. & Bush et al. (1990) \\
\hline La Pata & Brazil & -66.66 & 0.25 & 300 & $0-45000$ & TRFO & Lake & 12 & $2 \mathrm{C}$ & 1D & Digi & Colinvaux, $\mathrm{P}$. & $\begin{array}{l}\text { Colinvaux et al. } \\
\text { (1996); Colinvaux } \\
\text { et al. (2000) }\end{array}$ \\
\hline Lagoa das Patas & Brazil & -66.68 & 0.26 & 300 & $0-42210$ & TFFO & Lake & 16 & 1D & $1 \mathrm{C}$ & Raw & De Oliveira, P. E. & De Oliveira (1992) \\
\hline Piusbi & Colombia & -77.89 & 1.66 & 200 & $0-10400$ & TRFO & Lake & 3 & $1 \mathrm{C}$ & - & Raw & Behling, $\mathrm{H}$. & $\begin{array}{l}\text { Behling and } \\
\text { Hooghiemstra } \\
(1999)\end{array}$ \\
\hline Pitalito & Colombia & -76.50 & 1.75 & 1300 & $0-7350$ & WEFO & Mire & 6 & $6 \mathrm{D}$ & - & Raw & Bakker, J. G. M. & $\begin{array}{l}\text { van der Hammen et } \\
\text { al. (1980) }\end{array}$ \\
\hline
\end{tabular}


Table 3. Continued.

\begin{tabular}{|c|c|c|c|c|c|c|c|c|c|c|c|c|c|}
\hline Site & Country & Long. & Latitude & Alt. & Age range & Present biome & $\begin{array}{l}\text { Sample } \\
\text { type }\end{array}$ & $\mathrm{RC}$ & $\begin{array}{l}\text { DC at } 6000 \\
\text { yr BP }\end{array}$ & $\begin{array}{l}\text { DC at } 18000 \\
\text { yr BP }\end{array}$ & Data type & Analyst & Site publications \\
\hline Piagua & Colombia & -76.50 & 2.30 & 1700 & $0-14000$ & WEFO & Lake & 7 & $7 \mathrm{D}$ & - & Raw & Wille, M. & $\begin{array}{l}\text { Wille et al. (2001); } \\
\text { van der Hammen et al. } \\
\text { (1980) }\end{array}$ \\
\hline Pantano de Genagra & Colombia & -76.50 & 2.50 & 1750 & $0-9000$ & WEFO & Mire & 7 & $4 \mathrm{C}$ & - & Raw & Behling, $\mathrm{H}$. & $\begin{array}{l}\text { Behling et al. (1999); } \\
\text { van der Hammen et al. } \\
\text { (1980) }\end{array}$ \\
\hline Rio Timbio & Colombia & -76.50 & 2.50 & 1750 & $0-14000$ & WEFO & Lake & 6 & $2 \mathrm{C}$ & - & Raw & Wille, M. & $\begin{array}{l}\text { van der Hammen et al. } \\
\text { (1980) }\end{array}$ \\
\hline El Caimito & Colombia & -76.60 & 2.53 & 50 & $0-4500$ & TRFO & Lake & 4 & - & - & Raw & Wille, M. & Wille et al. (1999) \\
\hline Loma Linda & Colombia & -73.35 & 3.22 & 310 & $0-8720$ & TDFO & Lake & 8 & $1 \mathrm{C}$ & - & Raw & Behling, $\mathrm{H}$. & Behling et al. (1999) \\
\hline Lago Agua Sucia & Colombia & -73.54 & 3.46 & 260 & $0-15340$ & TDFO & Lake & 4 & $7 \mathrm{D}$ & - & Raw & Wijmstra, T. A. & $\begin{array}{l}\text { Wijmstra and van der } \\
\text { Hammen (1966) }\end{array}$ \\
\hline El Gobernador & Colombia & -75.00 & 3.95 & 3815 & $0-10050$ & CTRF & Mire & 2 & $2 \mathrm{C}$ & - & Raw & Melief, A. B. M. & Melief (1985) \\
\hline La Guitarra & Colombia & -74.28 & 4.00 & 3450 & $0-15300$ & COMI & Mire & 3 & $4 \mathrm{C}$ & - & Raw & Melief, A. B. M. & Melief (1985) \\
\hline La Primavera & Colombia & -74.13 & 4.00 & 3525 & $0-11200$ & CGSH & Mire & 6 & $1 \mathrm{C}$ & - & Raw & Melief, A. B. M. & Melief (1985) \\
\hline Corazón Partido & Colombia & -74.25 & 4.00 & 4100 & Modern & CGSH & Mire & - & - & - & Raw & Melief, A. B. M. & Melief (1985) \\
\hline El Trinagulo & Colombia & -74.25 & 4.00 & 4100 & Modern & CGSH & Mire & - & - & - & Raw & Melief, A. B. M. & Melief (1985) \\
\hline Carimagua & Colombia & -74.14 & 4.04 & 180 & $0-8270$ & TDFO & Lake & 6 & $2 \mathrm{C}$ & - & Raw & Behling, $\mathrm{H}$. & $\begin{array}{l}\text { Behling and Hooghiem- } \\
\text { stra (1999) }\end{array}$ \\
\hline La Rabona & Colombia & -74.25 & 4.05 & 4000 & $0-5100$ & CGSH & Mire & 1 & $4 \mathrm{D}$ & - & Raw & Melief, A. B. M. & Melief (1985) \\
\hline El Piñal & Colombia & -70.40 & 4.09 & 185 & $0-19000$ & TDFO & Lake & 8 & $4 \mathrm{C}$ & $2 \mathrm{D}$ & Raw & Behling, $\mathrm{H}$. & $\begin{array}{l}\text { Behling and Hooghiem- } \\
\text { stra (1999) }\end{array}$ \\
\hline Alsacia & Colombia & -74.11 & 4.09 & 3100 & $0-13700$ & COMI & Mire & 3 & $6 \mathrm{D}$ & - & Raw & Melief, A. B. M. & Melief (1985) \\
\hline Andabobos & Colombia & -74.15 & 4.09 & 3570 & $0-15000$ & CGSH & Mire & 2 & 7D & - & Raw & Melief, A. B. M. & Melief (1985) \\
\hline Ubaqué & Colombia & -73.55 & 4.33 & 2000 & Modern & WEFO & Lake & - & - & - & Raw & Jean-Jarcob, K. & $\begin{array}{l}\text { Wille et al. (2001); } \\
\text { van der Hammen et al. } \\
\text { (1980) }\end{array}$ \\
\hline de la América & Colombia & -74.00 & 4.33 & 3550 & $0-9000$ & CGSH & Mire & 1 & $1 \mathrm{D}$ & - & Raw & Kuhry, P. & $\begin{array}{l}\text { Kuhry (1988); van der } \\
\text { Hammen and González } \\
(1960)\end{array}$ \\
\hline Turbera de Calostros & Colombia & -73.48 & 4.41 & 3730 & Modern & CGSH & Soil & 1 & - & - & Raw & Salomons, J. B. & $\begin{array}{l}\text { van der Hammen et al. } \\
\text { (1980) }\end{array}$ \\
\hline Laguna Angel & Colombia & -70.54 & 4.45 & 205 & $0-10026$ & TDFO & Lake & 8 & $2 \mathrm{C}$ & - & Raw & Behling, $\mathrm{H}$. & $\begin{array}{l}\text { Behling and Hooghiem- } \\
\text { stra (1998) }\end{array}$ \\
\hline Libano & Colombia & -75.50 & 4.50 & 1820 & $0-14000$ & WEFO & Soil & 1 & $7 \mathrm{D}$ & - & Raw & Salomons, J. B. & $\begin{array}{l}\text { van der Hammen et al. } \\
\text { (1980) }\end{array}$ \\
\hline de Pedro Palo III & Colombia & -74.41 & 4.50 & 2000 & $0-5500$ & COMI & Lake & 2 & $7 \mathrm{D}$ & - & Raw & $\begin{array}{l}\text { van der Hammen, } \\
\text { T. }\end{array}$ & van der Hammen (1974) \\
\hline Paramo Palacio & Colombia & -73.88 & 4.76 & 3550 & $0-5500$ & CGSH & Mire & 4 & $5 \mathrm{D}$ & - & Raw & $\begin{array}{l}\text { van der Hammen, } \\
\text { T. }\end{array}$ & $\begin{array}{l}\text { van der Hammen and } \\
\text { González (1960) }\end{array}$ \\
\hline Greja & Colombia & -73.70 & 4.86 & 4000 & $0-12000$ & CGSH & Lake & 2 & $3 \mathrm{C}$ & - & Raw & $\begin{array}{l}\text { van der Hammen, } \\
\text { T. }\end{array}$ & van der Hammen (1962) \\
\hline Sardinas & Colombia & -69.45 & 4.95 & 180 & $0-11600$ & TDFO & Lake & 6 & $2 \mathrm{C}$ & - & Raw & Behling, $\mathrm{H}$. & $\begin{array}{l}\text { Behling and Hooghiem- } \\
\text { stra (1998) }\end{array}$ \\
\hline Herrera & Colombia & -73.91 & 5.00 & 2000 & 0-20000 & COMI & Lake & 3 & $4 \mathrm{D}$ & - & Raw & van Geel, B. & $\begin{array}{l}\text { van Geel and van der } \\
\text { Hammen (1973) }\end{array}$ \\
\hline Agua Blanca & Colombia & -74.45 & 5.0 & 3250 & $0-46000$ & COMI & Mire & 2 & $6 \mathrm{D}$ & $7 \mathrm{D}$ & Raw & Kuhry, P. & $\begin{array}{l}\text { Graf (1992); Kuhry } \\
\text { (1988b); Kuhry et al. } \\
(1983)\end{array}$ \\
\hline El Abra II & Colombia & -73.96 & 5.02 & 2570 & $0-11000$ & COMI & Cave & 1 & $7 \mathrm{D}$ & - & Raw & $\begin{array}{l}\text { Schreve-Brinkman, } \\
\text { E. J. }\end{array}$ & $\begin{array}{l}\text { Schreve-Brinkman } \\
\text { (1978) }\end{array}$ \\
\hline Paramo de Peña Negra & Colombia & -74.09 & 5.09 & 3625 & $0-12500$ & CGSH & Mire & 10 & $2 \mathrm{C}$ & - & Raw & Kuhry, P. & Kuhry et al. (1983) \\
\hline ODP site 932 & Brazil & -47.03 & 5.18 & 0 & $0-45000$ & TRFO & Fan & 2 & $2 \mathrm{C}$ & $1 \mathrm{D}$ & Raw & Haberle, S. & $\begin{array}{l}\text { Haberle and Maslin } \\
(1999)\end{array}$ \\
\hline Paramo de Laguna Verde & Colombia & -74.00 & 5.25 & 3625 & $0-5500$ & CGSH & Mire & 2 & $4 \mathrm{D}$ & - & Raw & Kuhry, P. & Kuhry et al. (1983) \\
\hline Fúquene II & Colombia & -73.87 & 5.50 & 2580 & $0-25000$ & COMI & Lake & 2 & $7 \mathrm{D}$ & $7 \mathrm{C}$ & Raw & van Geel, B. & $\begin{array}{l}\text { van Geel and van der } \\
\text { Hammen (1973) }\end{array}$ \\
\hline Jotaordó & Colombia & -76.66 & 5.66 & 50 & $0-4200$ & TRFO & Lake & 7 & - & - & Raw & Berrio, J. C. B. & Berrio et al. (2000) \\
\hline Cienaga del Visitador & Colombia & -72.83 & 6.13 & 3300 & $0-12000$ & COMI & Mire & 2 & $7 \mathrm{D}$ & - & Raw & $\begin{array}{l}\text { van der Hammen, } \\
\text { T. }\end{array}$ & $\begin{array}{l}\text { van der Hammen and } \\
\text { González (1965) }\end{array}$ \\
\hline Bobos & Colombia & -72.85 & 6.13 & 3800 & $0-5000$ & CGSH & Lake & 4 & $6 \mathrm{D}$ & - & Raw & $\begin{array}{l}\text { van der Hammen, } \\
\text { T. }\end{array}$ & van der Hammen (1962) \\
\hline Ciega I & Colombia & -72.31 & 6.50 & 3510 & $0-2000$ & COMI & Lake & 1 & - & - & Raw & $\begin{array}{l}\text { van der Hammen, } \\
\text { T. }\end{array}$ & $\begin{array}{l}\text { van der Hammen et al. } \\
\text { (1980) }\end{array}$ \\
\hline Valle de Lagunillas & Colombia & -72.34 & 6.50 & 3880 & $0-7100$ & CGSH & Lake & 8 & $7 \mathrm{D}$ & - & Raw & $\begin{array}{l}\text { van der Hammen, } \\
\text { T. }\end{array}$ & $\begin{array}{l}\text { van der Hammen et al. } \\
\text { (1980) }\end{array}$ \\
\hline Cana, Darien & Panama & -77.58 & 7.68 & 1000 & Modern & TSFO & Pollster & - & - & - & Raw & Bush, M. & $\begin{array}{l}\text { Bush (1995); Bush and } \\
\text { Rivera (1998) }\end{array}$ \\
\hline Cana Swamp & Panama & -77.59 & 7.74 & 500 & $0-4600$ & TRFO & Swamp & 5 & - & - & Digi & Bush, M. & $\begin{array}{l}\text { Bush and Colinvaux } \\
\text { (1994) }\end{array}$ \\
\hline Wodehouse Swamp & Panama & -77.58 & 7.75 & 500 & $0-4200$ & TRFO & Swamp & 1 & - & - & Digi & Bush, M. & $\begin{array}{l}\text { Bush and Colinvaux } \\
\text { (1994) }\end{array}$ \\
\hline La Chonta & Costa Rica & -82.00 & 8.00 & 2310 & $0-80000$ & CTRF & Lake & 3 & $2 \mathrm{C}$ & $7 \mathrm{D}$ & Digi & Islebe, $\mathrm{G}$. & $\begin{array}{l}\text { Hooghiemstra et al. } \\
(1992) ; \text { Islebe and } \\
\text { Hooghiemstra (1997); } \\
\text { Islebe et al. (1995a, b) }\end{array}$ \\
\hline
\end{tabular}


Table 3. Continued.

\begin{tabular}{|c|c|c|c|c|c|c|c|c|c|c|c|c|c|}
\hline Site & Country & Long. & Latitude & Alt. & Age range & Present biome & $\begin{array}{l}\text { Sample } \\
\text { type }\end{array}$ & $\mathrm{RC}$ & $\begin{array}{c}\mathrm{DC} \text { at } 6000 \\
\mathrm{yr} \mathrm{BP}\end{array}$ & $\begin{array}{l}\mathrm{DC} \text { at } 18000 \\
\mathrm{yr} \mathrm{BP}\end{array}$ & Data type & Analyst & Site publications \\
\hline El Valle & Panama & -79.78 & 8.43 & 500 & $0-20000$ & TSFO & Lake & 5 & - & $2 \mathrm{C}$ & Digi & Bush, M. & $\begin{array}{l}\text { Bush (1995); Bush and } \\
\text { Rivera (1998); Piperno et } \\
\text { al. (1991a, b) }\end{array}$ \\
\hline La Yeguada, & Panama & -80.78 & 8.43 & 650 & $0-14000$ & TSFO & Lake & 11 & $1 \mathrm{C}$ & - & Digi & Bush, M. & $\begin{array}{l}\text { Bush (1995); Bush et al. } \\
\text { (1992); Bush and Rivera } \\
(1998) \text {; Piperno et al. } \\
(1991 \text { a, b) }\end{array}$ \\
\hline Cerro Campana & Panama & -79.93 & 8.63 & 800 & Modern & WTFO & Pollster & - & - & - & Raw & Bush, M. & $\begin{array}{l}\text { Bush (1995); Bush and } \\
\text { Rivera (1998) }\end{array}$ \\
\hline Quebrada Nelson & Panama & -82.31 & 8.66 & 1130 & Modern & WTRF & Pollster & - & - & - & Raw & Bush, M. & $\begin{array}{l}\text { Bush (1995); Bush and } \\
\text { Rivera (1998) }\end{array}$ \\
\hline Laguna Volcán & Panama & -82.75 & 8.75 & 1500 & $0-2860$ & WTRF & Lake & 4 & - & - & Digi & Behling, $\mathrm{H}$. & Behling (2000) \\
\hline Valle Laguna Negra & Venezuela & -70.76 & 8.79 & 3450 & $0-3350$ & CGSH & Lake & 1 & - & - & Raw & Graf, K. & Rull et al. (1987) \\
\hline Valle Laguna Victoria & Venezuela & -70.79 & 8.80 & 3250 & $0-12210$ & CGSH & Lake & 4 & $4 \mathrm{C}$ & - & Raw & Graf, K. & Rull et al. (1987) \\
\hline Horsefly Ridge & Panama & -82.24 & 8.83 & 1150 & Modern & WTRF & Pollster & - & - & - & Raw & Bush, M. & $\begin{array}{l}\text { Bush (1995); Bush and } \\
\text { Rivera (1998) }\end{array}$ \\
\hline El Bosque & Colombia & -75.45 & 8.85 & 3650 & $0-4700$ & CGSH & Mire & 4 & - & - & Raw & Melief, A. B. M. & $\begin{array}{l}\text { Kuhry (1988a); Melief } \\
\text { (1985) }\end{array}$ \\
\hline Volcan Baru & Panama & -82.52 & 8.85 & 2600 & Modern & CTRF & Pollster & - & - & - & Raw & Bush, M. & $\begin{array}{l}\text { Bush (1995); Bush and } \\
\text { Rivera (1998) }\end{array}$ \\
\hline Finca Lerida & Panama & -82.45 & 8.87 & 1630 & Modern & WTRF & Pollster & - & - & - & Raw & Bush, M. & $\begin{array}{l}\text { Bush (1995); Bush and } \\
\text { Rivera (1998) }\end{array}$ \\
\hline Volcan Irazu & Panama & -82.52 & 8.88 & 2300 & Modern & CTRF & Pollster & - & - & - & Raw & Bush, M. & $\begin{array}{l}\text { Bush (1995); Bush and } \\
\text { Rivera (1998) }\end{array}$ \\
\hline Paramo de Miranda & Venezuela & -70.85 & 8.91 & 3290 & $310-11470$ & CGSH & Mire & 3 & $4 \mathrm{C}$ & - & Raw & $\begin{array}{l}\text { Salgado- } \\
\text { Labouriau, M. L. }\end{array}$ & $\begin{array}{l}\text { Salgado-Labouriau } \\
(1988,1991)\end{array}$ \\
\hline Panama & Panama & -80.87 & 9.00 & 100 & $0-7200$ & TSRO & Lake & 8 & $1 \mathrm{C}$ & - & Raw & Leyden, B. W. & Leyden et al. (1995) \\
\hline Ocelot Pond & Panama & -79.59 & 9.12 & 20 & Modern & TSFO & Pollster & - & - & - & Raw & Bush, M. & $\begin{array}{l}\text { Bush (1995); Bush and } \\
\text { Rivera (1998) }\end{array}$ \\
\hline Soberania & Panama & -79.66 & 9.13 & 20 & Modern & TSFO & Pollster & - & - & - & Raw & Bush, M. & $\begin{array}{l}\text { Bush (1995); Bush and } \\
\text { Rivera (1998) }\end{array}$ \\
\hline Paramo Piedras Blancas & Venezuela & -70.83 & 9.16 & 4080 & $0-1340$ & CGSH & Mire & 2 & - & - & Raw & $\begin{array}{l}\text { Salgado- } \\
\text { Labouriau, M. L. }\end{array}$ & Rull et al. (1987) \\
\hline Pipeline Rd & Panama & -79.66 & 9.33 & 40 & Modern & TSFO & Pollster & - & - & - & Raw & Bush, M. & $\begin{array}{l}\text { Bush (1995); Bush and } \\
\text { Rivera (1998) }\end{array}$ \\
\hline Barro Colorado Island & Panama & -79.75 & 9.35 & 50 & Modern & TSFO & Pollster & - & - & - & Raw & Bush, M. & $\begin{array}{l}\text { Bush (1995); Bush and } \\
\text { Rivera (1998) }\end{array}$ \\
\hline Lago Chirripó & Costa Rica & -83.48 & 9.48 & 3520 & Modern & CTRF & Lake & - & - & - & Digi & Horn, S. P. & $\begin{array}{l}\text { Rodgers and Horn } \\
\text { (1996) }\end{array}$ \\
\hline Talamancas & Costa Rica & -83.72 & 9.5 & 2500 & Modern & CTRF & Pollster & - & - & - & Raw & Bush, M. & $\begin{array}{l}\text { Bush (1995); Bush and } \\
\text { Rivera (1998) }\end{array}$ \\
\hline Lago de las Morrenas & Costa Rica & -83.49 & 9.50 & 3480 & $0-10000$ & CTRF & Lake & 6 & $2 \mathrm{C}$ & - & Digi & Horn, S. P. & Horn (1993) \\
\hline Bog 70 & Costa Rica & -83.85 & 9.61 & 2670 & Modern & CTRF & Bog & - & - & - & Digi & Horn, S. P. & $\begin{array}{l}\text { Rodgers and Horn } \\
\text { (1996) }\end{array}$ \\
\hline Tres de Junio & Costa Rica & -83.87 & 9.62 & 2670 & Modern & CTRF & Bog & - & - & - & Digi & Horn, S. P. & $\begin{array}{l}\text { Rodgers and Horn } \\
\text { (1996) }\end{array}$ \\
\hline Bog 68 & Costa Rica & -83.85 & 9.64 & 2670 & Modern & CTRF & Bog & - & - & - & Digi & Horn, S. P. & 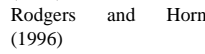 \\
\hline Asuncion & Costa Rica & -83.75 & 9.64 & 3340 & Modern & CTRF & Lake & - & - & - & Digi & Horn, S. P. & $\begin{array}{l}\text { Rodgers and Horn } \\
\text { (1996) }\end{array}$ \\
\hline Carara Biological Reserve & Costa Rica & -84.62 & 9.73 & 0 & Modern & TSFO & Pollster & - & - & - & Raw & Bush, M. & $\begin{array}{l}\text { Bush (1995); Bush and } \\
\text { Rivera (1998) }\end{array}$ \\
\hline Quebrador & Costa Rica & -83.84 & 9.74 & 3040 & Modern & CTRF & Lake & - & - & - & Digi & Horn, S. P. & $\begin{array}{l}\text { Rodgers and Horn } \\
\text { (1996) }\end{array}$ \\
\hline Cataracta, Carara 1 & Costa Rica & -84.63 & 9.83 & 270 & Modern & TSFO & Pollster & - & - & - & Raw & Bush, M. & $\begin{array}{l}\text { Bush (1995); Bush and } \\
\text { Rivera (1998) }\end{array}$ \\
\hline Cataracta, Carara 2 & Costa Rica & -84.63 & 9.85 & 270 & Modern & TSFO & Pollster & - & - & - & Raw & Bush, M. & $\begin{array}{l}\text { Bush (1995); Bush and } \\
\text { Rivera (1998) }\end{array}$ \\
\hline Carara & Costa Rica & -84.60 & 9.88 & 35 & Modern & TSFO & Lake & - & - & - & Digi & Horn, S. P. & $\begin{array}{l}\text { Rodgers and Horn } \\
(1996)\end{array}$ \\
\hline Laguna Bonilla & Costa Rica & -83.61 & 9.99 & 380 & Modern & TSFO & Lake & - & - & - & Digi & Horn, S. P. & $\begin{array}{l}\text { Rodgers and Horn } \\
\text { (1996) }\end{array}$ \\
\hline Laguna Barva & Costa Rica & -84.11 & 10.14 & 2840 & Modern & CTRF & Lake & - & - & - & Digi & Horn, S. P. & 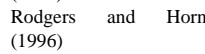 \\
\hline Laguna Palmita & Costa Rica & -84.95 & 10.18 & 60 & Modern & TDFO & Soil & - & - & - & Digi & Horn, S. P. & $\begin{array}{l}\text { Rodgers and Horn } \\
\text { (1996) }\end{array}$ \\
\hline Laguna Botos & Costa Rica & -84.18 & 10.18 & 2600 & Modern & CTRF & Lake & - & - & - & Digi & Horn, S. P. & 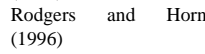 \\
\hline Bosque Alegre & Costa Rica & -84.21 & 10.21 & 740 & Modern & WTRF & Lake & - & - & - & Digi & Horn, S. P. & $\begin{array}{l}\text { Rodgers and } \\
(1996)\end{array}$ \\
\hline Laguna González & Costa Rica & -84.45 & 10.25 & 710 & Modern & WTRF & Lake & - & - & - & Digi & Horn, S. P. & $\begin{array}{l}\text { Rodgers and Horn } \\
\text { (1996) }\end{array}$ \\
\hline Laguna Congo & Costa Rica & -84.29 & 10.27 & 740 & Modern & WTRF & Lake & - & - & - & Digi & Horn, S. P. & $\begin{array}{l}\text { Rodgers and } \\
(1996)\end{array}$ \\
\hline Laguna Hule & Costa Rica & -84.19 & 10.27 & 740 & Modern & WTRF & Lake & - & - & - & Digi & Horn, S. P. & $\begin{array}{l}\text { Rodgers and Horn } \\
\text { (1996) }\end{array}$ \\
\hline Laguna María Aguilar & Costa Rica & -84.18 & 10.27 & 770 & Modern & WTRF & Lake & - & - & - & Digi & Horn, S. P. & $\begin{array}{l}\text { Rodgers and Horn } \\
\text { (1996) }\end{array}$ \\
\hline Brauillo Carillo, Heredia & Costa Rica & -83.94 & 10.3 & 630 & Modern & WTRF & Pollster & - & - & - & Raw & Bush, M. & $\begin{array}{l}\text { Bush (1995); Bush and } \\
\text { Rivera (1998) }\end{array}$ \\
\hline Monteverde, Heredia & Costa Rica & -84.8 & 10.3 & 1500 & Modern & WTRF & Pollster & - & - & - & Raw & Bush, M. & $\begin{array}{l}\text { Bush (1995); Bush and } \\
\text { Rivera (1998) }\end{array}$ \\
\hline Volcan Poas & Costa Rica & -84.19 & 10.3 & 2580 & Modern & CTRF & Pollster & - & - & - & Raw & Bush, M. & $\begin{array}{l}\text { Bush (1995); Bush and } \\
\text { Rivera (1998) }\end{array}$ \\
\hline Lake Valencia & Venezuela & -67.75 & 10.32 & 403 & $400-13000$ & STEP & Lake & 28 & $1 \mathrm{C}$ & - & Raw & Leyden, B. W. & $\begin{array}{l}\text { Bradbury et al. (1981); } \\
\text { Leyden (1985) }\end{array}$ \\
\hline Laguna Río Cuarto & Costa Rica & -84.18 & 10.34 & 380 & Modern & WTRF & Lake & - & - & - & Digi & Horn, S. P. & 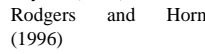 \\
\hline
\end{tabular}


Table 3. Continued.

\begin{tabular}{|c|c|c|c|c|c|c|c|c|c|c|c|c|c|}
\hline Site & Country & Long. & Latitude & Alt. & Age range & Present biome & $\begin{array}{c}\text { Sample } \\
\text { type }\end{array}$ & $\mathrm{RC}$ & $\begin{array}{c}\text { DC at } 6000 \\
\text { yr BP }\end{array}$ & $\begin{array}{c}\text { DC at } 18000 \\
\text { yr BP }\end{array}$ & Data type & Analyst & Site publications \\
\hline La Selva, Heredia & Costa Rica & -84.00 & 10.43 & 80 & Modern & TSFO & Pollster & - & - & - & Raw & Bush, M. & $\begin{array}{l}\text { Bush (1995); Bush } \\
\text { and Rivera (1998) }\end{array}$ \\
\hline Cantarrana Swamp & Costa Rica & -84.00 & 10.45 & 36 & Modern & TRFO & Swamp & - & - & - & Digi & Horn, S. P. & $\begin{array}{l}\text { Rodgers and Horn } \\
\text { (1996) }\end{array}$ \\
\hline La Pacifica, Guanacaste & Costa Rica & -85.11 & 10.45 & 110 & Modern & TSFO & Pollster & - & - & - & Raw & Bush, M. & $\begin{array}{l}\text { Bush (1995); Bush } \\
\text { and Rivera (1998) }\end{array}$ \\
\hline Sendro Sedro Swamp & Costa Rica & -84.00 & 10.46 & 40 & Modern & TRFO & Swamp & - & - & - & Digi & Horn, S. P. & $\begin{array}{l}\text { Rodgers and Horn } \\
\text { (1996) }\end{array}$ \\
\hline Laguna La Palma & Costa Rica & -84.73 & 10.49 & 570 & Modern & WTRF & Lake & - & - & - & Digi & Horn, S. P. & $\begin{array}{l}\text { Rodgers and Horn } \\
\text { (1996) }\end{array}$ \\
\hline Laguna Cedeño & Costa Rica & -84.71 & 10.49 & 610 & Modern & WTRF & Lake & - & - & - & Digi & Horn, S. P & $\begin{array}{l}\text { Rodgers and Horn } \\
\text { (1996) }\end{array}$ \\
\hline Tortuguero & Costa Rica & -83.53 & 10.53 & 0 & Modern & TSFO & Pollster & - & - & - & Raw & Bush, M. & $\begin{array}{l}\text { Bush (1995); Bush } \\
\text { and Rivera (1998) }\end{array}$ \\
\hline Santa Rosa2 & Costa Rica & -85.64 & 10.83 & 0 & Modern & TSFO & Soil & - & - & - & Digi & Horn, S. P. & $\begin{array}{l}\text { Rodgers and Horn } \\
\text { (1996) }\end{array}$ \\
\hline Santa Rosa1 & Costa Rica & -85.66 & 10.84 & 0 & Modern & TRFO & Soil & - & - & - & Digi & Horn, S. P. & $\begin{array}{l}\text { Rodgers and Horn } \\
\text { (1996) }\end{array}$ \\
\hline Boca de Lopez & Colombia & -75.36 & 10.85 & 0 & $0-4000$ & TRFO & Coastal & 5 & - & - & Raw & $\begin{array}{l}\text { van der Hammen, } \\
\text { T. }\end{array}$ & $\begin{array}{l}\text { Behling et al. } \\
\text { (1999) }\end{array}$ \\
\hline Cafetal, Guanacaste & Costa Rica & -85.65 & 10.85 & 300 & Modern & TSFO & Pollster & - & - & - & Raw & Bush, M. & $\begin{array}{l}\text { Bush (1995); Bush } \\
\text { and Rivera (1998) }\end{array}$ \\
\hline Santa Rosa3 & Costa Rica & -85.62 & 10.86 & 280 & Modern & TSFO & Soil & - & - & - & Digi & Horn, S. P. & $\begin{array}{l}\text { Rodgers and Horn } \\
\text { (1996) }\end{array}$ \\
\hline Santa Rosa4 & Costa Rica & -85.62 & 10.86 & 280 & Modern & TSFO & Soil & - & - & - & Digi & Horn, S. P. & $\begin{array}{l}\text { Rodgers and Horn } \\
\text { (1996) }\end{array}$ \\
\hline Escondido & Costa Rica & -85.61 & 10.87 & 280 & Modern & TSFO & Lake & - & - & - & Digi & Horn, S. P. & $\begin{array}{l}\text { Rodgers and Horn } \\
\text { (1996) }\end{array}$ \\
\hline Volcán Cacao & Costa Rica & -85.47 & 10.92 & 1000 & Modern & WTRF & Soil & - & - & - & Digi & Horn, S. P. & $\begin{array}{l}\text { Rodgers and Horn } \\
\text { (1996) }\end{array}$ \\
\hline Sierra de Cuchumatanes5 & Guatemala & -91.00 & 15.75 & 2800 & Modern & WAMF & Polster & - & - & - & Digi & Islebe, $\mathrm{G}$. & $\begin{array}{l}\text { Islebe and } \\
\text { Hooghiemstra } \\
(1995)\end{array}$ \\
\hline Sierra de Cuchumatanes 4 & Guatemala & -91.25 & 15.75 & 3000 & Modern & CTRF & Polster & - & - & - & Digi & Islebe, G. & $\begin{array}{l}\text { Islebe and } \\
\text { Hooghiemstra } \\
(1995)\end{array}$ \\
\hline Sierra de Cuchumatanes3 & Guatemala & -91.5 & 15.75 & 3400 & Modern & CTRF & Polster & - & - & - & Digi & Islebe, $\mathrm{G}$. & $\begin{array}{l}\text { Islebe and } \\
\text { Hooghiemstra } \\
(1995)\end{array}$ \\
\hline Sierra de Cuchumatanes2 & Guatemala & -91.75 & 15.75 & 3600 & Modern & WAMF & Polster & - & - & - & Digi & Islebe, $\mathrm{G}$. & $\begin{array}{l}\text { Islebe and } \\
\text { Hooghiemstra } \\
\text { (1995) }\end{array}$ \\
\hline Sierra de Cuchumatanes 1 & Guatemala & -92.00 & 15.75 & 4200 & Modern & CGSH & Polster & - & - & - & Digi & Islebe, G. & $\begin{array}{l}\text { Islebe and } \\
\text { Hooghiemstra } \\
(1995)\end{array}$ \\
\hline Lago Quexil & Guatemala & -89.88 & 16.92 & 110 & $0-27500$ & TSFO & Lake & 4 & $7 \mathrm{D}$ & - & Raw & Leyden, B. W. & $\begin{array}{l}\text { Leyden (1984); } \\
\text { Leyden et al. } \\
(1993,1994)\end{array}$ \\
\hline Lake Peten-Itza & Guatemala & -90.00 & 17.25 & 200 & $0-9000$ & TDFO & Lake & 7 & $2 \mathrm{C}$ & - & Digi & Islebe, G. & $\begin{array}{l}\text { Islebe et al. } \\
\text { (1996) }\end{array}$ \\
\hline Lago Catemaco & Mexico & -95.00 & 18.66 & 340 & $0-2230$ & TRFO & Lake & 5 & $2 \mathrm{C}$ & - & Raw & Byrne, A. R. & $\begin{array}{l}\text { Byrne and Horn } \\
\text { (1989) }\end{array}$ \\
\hline Zempoala & Mexico & -99.30 & 19.20 & 3100 & $0-4600$ & WAMF & Lake & 5 & - & - & Raw & Almeida, L. & Almeida (1997) \\
\hline Quila & Mexico & -99.20 & 19.30 & 2800 & $0-10000$ & WAMF & Lake & 4 & $3 \mathrm{C}$ & - & Raw & Almeida, L. & Almeida (1997) \\
\hline Lake Texcoco & Mexico & -99.12 & 19.44 & 2330 & $0-35000$ & WAMF & Lake & 7 & $3 \mathrm{C}$ & $4 \mathrm{C}$ & Digi & Lozano-García, S. & $\begin{array}{l}\text { Lozano-García and } \\
\text { Ortega-Guerrero } \\
\text { (2009) }\end{array}$ \\
\hline Chalco Lake & Mexico & -99.00 & 19.50 & 2240 & $8000-27500$ & WAMF & Lake & 8 & $3 \mathrm{C}$ & $1 \mathrm{C}$ & Raw & $\begin{array}{l}\text { Lozano-Garcia, } \\
\text { M. S. }\end{array}$ & $\begin{array}{l}\text { Lozano-Garcia and } \\
\text { Ortega-Guerrero } \\
\text { (1994); Lozano- } \\
\text { Garcia et al. } \\
\text { (1993); Ortega- } \\
\text { Guerrero (1992) }\end{array}$ \\
\hline Lake Pátzcuaro & Mexico & -101.58 & 19.58 & 2044 & $20-44100$ & WAMF & Lake & 24 & $2 \mathrm{C}$ & $6 \mathrm{C}$ & Raw & Watts, W. A. & $\begin{array}{l}\text { Saporito (1975), } \\
\text { Watts and Bradbuy } \\
\text { (1982) }\end{array}$ \\
\hline San Jose Chulchaca & Mexico & -90.13 & 20.86 & 1 & $0-7300$ & TDFO & Lake & 8 & $2 \mathrm{C}$ & - & Raw & Leyden, B. W. & $\begin{array}{l}\text { Leyden et al. } \\
\text { (1995) }\end{array}$ \\
\hline Lake Coba & Mexico & -87.55 & 20.86 & 100 & $5880-19230$ & WAMF & Playa & 8 & $7 \mathrm{D}$ & $2 \mathrm{C}$ & Raw & Leyden, B. W. & $\begin{array}{l}\text { Leyden et al. } \\
\text { (1998) }\end{array}$ \\
\hline
\end{tabular}

that have been used to unravel past vegetation changes in Latin America with ecosystem reconstructions now existing from all major vegetation types over the late Quaternary period. The Latin American Pollen Database (LAPD) (www.ncdc.noaa.gov/paleo/lapd.html) is an online resource used to collate these data and facilitated the systematic interoperation presented here; indeed the majority of the pollen data used here are available through the LAPD. Additional data were obtained from researchers working in Latin America; all active palynolgists being given the opportunity to contribute data not currently lodged in the LAPD. Indeed, data from a number of sites in Argentina, Brazil, Costa Rica, México and Panama were made available specifically for this work. The majority of data from Colombia were prepared for 
this analysis directly from the original count sheets and are in preparation for uploading to the LAPD.

The majority of the data used in our analysis are complete raw pollen counts, this permitted all pollen taxa recorded by the original analyst to be allocated to PFTs and allowed the integrity of the data to be maintained throughout the analysis. Application of raw pollen data in other regions has been shown to help in differentiating between biomes (Tarasov et al., 1998b). However, numerous pollen records are either not submitted to the LAPD, or, were not made available for this analysis. Rather than omitting these data, the pollen counts were digitised from published pollen diagrams (Table 3): digitising such data provides a spatially more complete reconstruction than available from presented archived data. The process of digitisation involved either back calculation of the pollen counts if information on the pollen sum was present; if the pollen sum was not available the pollen percentage diagram was used as a count of 100 and values for the pollen taxa were abstracted at the time intervals used for our analysis. This scenario of combining data from different formats comes with a number of caveats that can have bearing on the results, and their interpretation (Marchant and Hooghiemstra, 2001). Firstly, the sub-set of pollen taxa in a count used to construct published pollen diagrams, and pollen sums that comprise it, often result from the bias of individual researchers', particularly on what are the reliable indicator taxa for a particular area and range of different vegetation types under investigation. This issue is particularly crucial in Latin America where the large numbers of pollen taxa encountered in the original counting are rarely depicted on published pollen diagrams. Furthermore, the level of identification achieved within pollen analysis, to a generic or family level, commonly comprises species that can be found in a range of different vegetation types, ecologies and growth forms (Marchant et al., 2002c). The majority of the samples for the biomisation presented here are derived from sites close to the Andean spine. Primarily, this concentration reflects the sensitive response of the vegetation to climate change on the steep altitudinal gradients (Marchant et al., 2001b); the area forming an ideal location for palaeoecological research. Additionally, the comparative lack of data from the lowlands is fuelled by problems of access, suitable sites and strong river dynamics that commonly result in sedimentary hiatuses (Ledru, 1998). Because of the steep environmental gradients associated with $7000 \mathrm{~m}$ of altitudinal change found along the Andes this spatial bias did not reduce the number of biomes we were able to reconstruct, (Fig. 4). However, the concentration of sites did result in numerous assignments being mapped overlapping each other and hence the changes between the different time periods difficult to detect. To rectify this problem in addition to presenting the results on the traditional biome dot maps (Figs. 7, 8 and 9) results are presented for all sites in a table (Table 6) that has been ordered by altitude so one can see which biome has been reconstructed for each site, how this compares to the potential vegetation and how it changes at each time period.

Uncalibrated radiocarbon dates available from the original stratigraphic analysis were used to select samples representing the time period used here. On a site-by-site basis, a linear age-depth model was applied to the pollen data. The validity of this model was assessed at each site taking into account sedimentary hiatuses and dating problems such as age reversals and dates with large standard errors; a summary of this dating control is provided in Table 1 following the COHMAP scheme (Webb, 1995; Yu and Harrison, 1999). Multiple samples $(\leq 3)$ were selected when more than one sample fell within the age range allowed for each time period. These data were compiled, to produce a site $v s$ taxa matrix that was then checked to standardise nomenclature, e.g., the combined file contained many synonyms such as Gramineae and Poaceae, and Mysine and Rapanea. Synonymous taxa were combined using the nomenclature of Kewensis (1997) and the International Plant Names Index (IPNI) (2008). Aquatic and non-tree fern taxa were removed from the matrix as they commonly reflect local hydrological conditions rather than local climate envelope. Marker additions and exotic spikes such as Lycopodium were also removed.

A total of 381 samples from 287 locations derived from core tops $\left(<500{ }^{14} \mathrm{C}\right.$ yr BP $)$, surface samples, pollen traps and moss polsters comprise the modern data set (Table 3). For the time period $6000 \pm 500{ }^{14} \mathrm{C}$ yr BP, 255 samples derived from 127 pollen records comprise the data set (Table 3 ). For the time period $18000 \pm 1000{ }^{14} \mathrm{C}$ yr BP, 61 samples derived from 34 pollen records comprise the data set (Table 3 ). The data sets to undergo analysis comprised 515 pollen taxa for the modern calibration, 493 for the $6000{ }^{14} \mathrm{C}$ yr BP reconstruction and 232 for the $18000{ }^{14} \mathrm{C}$ yr BP reconstruction. The taxonomic diversity of the Neotropical phytogeographical realm is greater than Africa [364] (Jolly et al., 1998a), Europe [41] (Prentice et al., 1996b), Russia and Mongolia [98] (Tarasov et al., 1998a) and China [68] (Yu et al., 1998), however this number will be biased by sampling intensity and density and some of these previous applications only focuses on the numerically important taxa.

\subsection{Biomisation}

Prentice et al. (1996a) and Prentice and Webb (1998) have documented the steps involved in the biomisation technique. First, a conceptual framework for PFTs and biomes in Latin American vegetation was developed by investigating the relationship between potential biomes and three environmental gradients. The environmental gradients considered were moisture availability ( $\alpha$ : Priestley-Taylor coefficient of plant available moisture), temperature (MTCO: mean temperature of the coldest month) and seasonal warmth (GDD: growing degree-days). Similarly to the biomes, but at a finer ecological resolution, the spatial distribution of PFTs is determined by environmental controls on plant growth form and ecological tolerance (Woodward, 1987). In Latin America the 
dominant environmental gradients are temperature, primarily associated with altitude, moisture availability and seasonality. PFT definitions were modified from the classification originally developed for the BIOME 1 model (Prentice et al., 1992, 1996a, b) taking into account schemes developed for other regions, particularly those that abut the Latin American region or contain similar floristic elements (Jolly et al., 1998a; Pickett et al., 2004; Takahara et al., 2001 Elenga et al., 2000; Thompson and Anderson, 2000; Yu et al., 2000). Five main groups of PFT were distinguished: these containing tropical (non-frost tolerant), coniferous (needle-leaved), temperate (frost tolerant), xerophytic (drought tolerant), and frost and drought tolerant taxa (Table 1, Fig. 6). This latter group is present in cold dry conditions of southern South America and the high Andes. A sixth "miscellaneous" group represents various life forms with restricted diagnostic value. The Latin American flora was divided into 25 PFTs (Table 1) that, although being of ecologically distinct, can be multiply assigned to the biomes (Table 2). The classification is based on the original scheme devised for the Biome1 vegetation model (Prentice et al., 1992) and modification through regional applications to pollen data. Where possible the scheme devised for Latin America conforms to existing classification and definitions. However, some of the specific vegetation types in Latin America were not adequately covered by the existing range so two new PFTs (heath and cushion plants) were added. To aid in the separation of the African forest/savanna boundary, Jolly et al. (1998a) subdivided the tropical raingreen trees PFT (Tr) into three groups. In the case of Latin America, it was decided that the overlap (taxa being multiply assigned to the PFTs) between the PFTs would be too great, and the distinction somewhat minimal. Furthermore, the tropical xerophytic trees and shrubs PFT encompass many taxa that would be assigned to the driest tropical raingreen category. Therefore, the Tr PFT was subdivided into "wet" $\left(\operatorname{Tr}_{1}\right)$ and "dry" $\left(\operatorname{Tr}_{2}\right)$ tropical raingreen trees.

The cornerstone of research concerned with the composition and distribution of Latin American vegetation, be it in a contemporary time frame, or one that aims to work in the past, is a good understanding of the ecology and distribution of the taxa concerned. The Latin American pollen taxa were assigned to one or more PFTs depending on the modern ecological range of the most important (i.e. most abundant) taxa responsible for producing the pollen identifiable within the modern data set. These assignments were made following reference to the known biology of plants from several floras (Rzedowski, 1983; Schofield, 1984; Wingenroth and Suarez, 1984; Kahn and de Granville, 1992; Gentry, 1993; Maberly, 1993; Seibert, 1996), botanical and palynological studies (Beard, 1955; van der Hammen, 1963; 1972; Wijmstra and van der Hammen, 1966; Eiten, 1972; Cleef and Hooghiemstra, 1984; Hooghiemstra and Cleef, 1984; Pires and Prance, 1985; Prance, 1985; Cuatrecasas and Barreto, 1988; Brown and Lugo, 1990; Bush, 1991; Dov Par, 1992; Kappelle, 1993;
Duivenvoorden and Cleef, 1994; Witte, 1994; Armesto et al., 1995; Harley, 1995; Kappelle, 1995; Kershaw and McGlone, 1995; Veblen et al., 1995; Colinvaux, 1996; Grabherr, 1997; Hooghiemstra and van der Hammen, 1998) and personal communication with modern ecologists and palaeoecologists. Much of this information has been collated into a dictionary on the distribution and ecology of parent taxa of pollen lodged within the Latin American Pollen Database (Marchant et al., 2002c). The resultant taxon vs. PFT assignments are presented in Table 4. Due to the high intra-generic diversity, and also the wide range of ecology's exhibited by the parent taxa present within some genera, a number of taxa were multiply assigned to a number of PFTs; where possible, pollen taxa were assigned to the PFTs within which the parent taxa are most common.

Thus, the identified PFTs from Latin America are described by the suite of pollen taxa assigned to them, in turn the biomes are distinguished by the suite of constituent PFTs (Table 5). A number of pollen taxa belong to more than one PFT (Table 4), and, as is the case with the potential vegetation, most PFTs contribute to more than one biome (Table 5). Two problems can arise here for our analysis that can be circumvented by manipulation of the input matrices and output biome affinity scores (the numerical sum computed for the pollen spectra for each sample this is equal to the sum of the square root of the percentages of the pollen types). First, pollen samples can have equal maximum affinity with more than one biome; this commonly occurs when the PFTs characteristic of one biome are a subset of another biome. Assigning the biomes so that subsets always come first in the analysis solves the problem. A second problem arises where multiple samples fall within the time window we are interested in. Multiple samples from a single site may have maximum affinity to a number of different biomes; the chance of this is high when the score of the "best" biome is close to that of the next "best" and the dominant biome switches between the top scoring biomes. In such cases, the "majority" biome is mapped. For example, site A contains eight samples within the time frame of $6000 \pm 500{ }^{14} \mathrm{C}$ yr BP, five samples have the greatest affinity to biome 1 , two samples to biome 2 and one sample to biome 3. The result is that biome 1 is mapped for site A at $6000 \pm 500{ }^{14} \mathrm{C}$ yr BP. This situation is not ideal as it masks the transient nature of the ecosystem to environmental shifts, however, this is a problem with the biomisation technique and can be overcome at a single site or few sites by mapping the biome scores rather than the most dominant biome (Marchant et al 2002a, b).

To enable a definition of the biomes to be based on bioclimatic data the climate space encompassed by Latin America (as abstracted from the climate data set of Leemans and Cramer, 1991) was used to plot plant available moisture $(\alpha)$ against a temperature gradient (MTCO). This plot was used to map the locational range of the biomes and to identify the location of the sites used in the analysis where there are pollen data for each of the three time periods in 
two-dimensional bioclimatic space (Fig. 5). Two immediate issues are apparent from this plot - firstly there is a bias of sites with an $\alpha$ of 1 within Latin America and a potentially different measure of moisture could provide some definition in this area of the plot. Secondly, as would be expected, there is little desert bioclimatic space in Latin America and no pollen sites located in this area of the plot. The twelve biomes identified within Latin America (Table 2) are designed to incorporate the range of major vegetation types and ensure consistency with previous areas to undergo the process within the BIOME-6000 community. Biomes were reconstructed from pollen data at sites with surface sample, trap and radiocarbon-dated core-top data. The results were used to produce a modern pollen-derived biome dot map (Fig. 7); for each site a colour dot records the reconstructed biome with the highest affinity score. These were compared site by site, with the potential modern vegetation distribution (Fig. 2). The biomisation procedure was applied to the fossil datasets without modification. Results for all sites and periods are provided in Table 6 which allows a site-by-site comparison through time and a comparison between the modern reconstruction and potential vegetation.

\section{Results}

\subsection{Modern pollen vs. potential biome reconstruction}

Visual comparison shows that the biomes reconstructed from modern pollen data (Fig. 7) reflect the broad features in the potential vegetation map (Fig. 2). In particular the modern reconstruction reproduces the transition from relatively mesic vegetation types, around the coastal areas of South America, to the more xeric biomes towards the interior. For example, in eastern Argentina there is a transition from steppe to xerophytic woods and scrub. Warm temperate rain forest is an important biome in the southern and southeastern Brazilian highlands, with tropical dry forest being reconstructed towards the interior. Notable from this region is the large number of different biomes being reconstructed in a relatively small area. In part this reflects the variability of potential vegetation, not portrayed in our relatively coarse resolution vegetation map (Fig. 2). For example, a site recording tropical rain forest reflects the sites' lowland position where it is characterised by moist gallery forest with a number of typical rain forest taxa present. Steppe is correctly reconstructed from the grasslands of south-eastern Argentina and dry forest in central Argentina, mirroring the transition to "drought-deciduous thorn forests" of central Argentina (Schmithüsen, 1976). Steppe is assigned farther west at approximately $1000 \mathrm{~m}$ in the Andes, southernmost South America and northeast Brazil. The vegetation of southern South America is dominated by cool temperate rain forest. The failure of the analysis to pick up the transition from cool temperate rain forest to cool grass/shrubland as one progresses east along Tierra del Fuego stems from the pollen spectra having a relatively large amount of Nothofagus pollen. Moving northwards from southern South America there is a transition to cool mixed forest, cool grass/shrubland and steppe; these latter assignments are particularly associated with eastern flanks of the Cordillera de los Andes.

The concentration of sites along the Andes results in a wide range of reconstructed biomes being geographically adjacent to each other when mapped in two-dimensional space (Fig. 7). This phenomenon is most apparent in the northern Andes where the altitudinal, and therefore climatic gradients are at their steepest. Despite these rapid environmental changes, the biome assignments reflect the changing vegetation patterns (Table 6). There is an altitudinal transition with low altitudes $(<300 \mathrm{~m})$ being mainly assigned to the tropical rain forest, tropical dry forest, tropical seasonal forest and steppe. Sites located at mid altitudes are described by a number of different biomes including tropical seasonal forest, warm mixed forest, cool mixed forest and cool temperate rain forest. Within this wide range of warm temperate rain forest and tropical seasonal forest are commonly assigned at lower elevations (Fig. 7, Table 6). Many of the sites at high altitude have a high affinity to the cool grass/shrubland biome. The line of the Andes can be easily seen by the cool grass/shrubland biome assignments, these being commonly recorded at sites above $3800 \mathrm{~m}$. The warm temperate rain forest is assigned at lower elevations and is analogous to Andean forest, being dominated by Podocarpus, Quercus and Weinmannia comprises a different forest type to that assigned in southern and southeast Brazil or southern South America. A mixture of biomes presently characterises the Amazon Basin with only four sites recording the tropical rain forest biome; two of these are in coastal locations. Tropical seasonal forest is recorded in four locations; this representing a slightly drier type of forest than tropical rain forest, containing some deciduous taxa. A number of "Amazonian" sites record warm temperate rain forest, these assignments responding to the presence of Andean floristic elements within lowland vegetation. There are a number of sites that record tropical dry forest, this being relatively widespread, e.g. on Easter Island, lowland Colombia and the Brazilian interior. Warm temperate rain forest describes the majority of the sites in the Panamanian and southern Costa Rican isthmus with warm mixed forest, being commonly at higher altitudes. This is an area where the comparison between the observed and predicted biomes shows a discrepancy; the possible reasons behind this will be discussed fully. Warm mixed forest is correctly assigned to the highlands of central and southern México as is tropical dry forest in southern México.

Investigating the correspondence between the pollenbased reconstruction and the potential vegetation for individual biomes provides a check on the methodology, particularly the construction of the matrices. The cool grass/shrubland biome is accurately reconstructed at the majority of sites. The sites that do not match the potential 
Table 4. Latin American pollen taxa used in the biomisation analysis and their assignment to the plant functional types (PFTs).

\begin{tabular}{|c|c|}
\hline PFT Codes & Pollen Taxa \\
\hline g & Poaceae. \\
\hline man & Acrostichum-type, Avicennia, Laguncularia, Rhizophora. \\
\hline tx & Alsophila, Alstroemeria, Cnemidaria, Cyathea, Dicksonia, Nephelea. \\
\hline $\operatorname{Tr}_{1}$ & $\begin{array}{l}\text { Alibertia, Anacardiaceae, Andira-type, Astronium, Bauhinia, Bombacaceae, Bougainvillea, Caesalpineae, Casearia- } \\
\text { type, Clematis, Coccoloba, Copaifera, Didymopanax, Eugenia, Euterpe, Goupia-type, Guapira, Heliocarpus, Hura, } \\
\text { Hieronima, Hippocrateaceae, Ipomoea, Laplacea, Lecythidaceae, Leguminoseae, Loranthaceae, Macharium, Macrolo- } \\
\text { bium, Malpighiaceae, Malvaceae, Maytenus, Ocotea-type, Pera, Phyllostylon, Piper, Pisonia, Psychotria, Rubiaceae, } \\
\text { Rutaceae, Salix, Sapium, Simira, Siparuna, Spirotheca, Spondias, Symplocos, Tecoma, Trema, Xylosma, Zanthoxylum. }\end{array}$ \\
\hline $\operatorname{Tr}_{2}$ & $\begin{array}{l}\text { Acacia, Alchornea, Alibertia, Andira-type, Anacardiaceae, Apocynaceae, Astronium, Banisteriopsis, Bauhinia, Bom- } \\
\text { bacaceae, Bougainvillea, Brosimum, Brunellia, Bulnesia, Bumelia-type, Bursera, Byrsonima, Caesalpineae, Celas- } \\
\text { traceae, Chrysophyllum, Clematis, Combretaceae, Copaifera, Cordia, Coriaria, Cuphea, Curatella, Didymopanax, } \\
\text { Elaeocarpaceae, Eryngium, Erythrina, Euterpe, Gallesia, Guapira, Hieronima, Hura, Hymenophylleace, Ipomoea, Lo- } \\
\text { ranthaceae, Malvaceae, Maytenus, Melastomataceae, Meliaceae, Mimosa, Passiflora, Pera, Phyllostylon, Piper, Piso- } \\
\text { nia, Protium, Pseudobombax, Rhamnaceae, Rutaceae, Salix, Sapium, Schinus, Simira, Siparuna, Spirotheca, Spondias, } \\
\text { Styrax, Symplocos, Tiliaceae, Trema, Xylosma, Zanthoxylum. }\end{array}$ \\
\hline
\end{tabular}

$\mathrm{Te}_{1} \quad$ Abutilon, Actinostemon concolor, Alchornea, Amanoa, Apeiba, Apocynaceae, Araliaceae, Arecaceae, Arrabidaea, Aspidosperma, Astrocarium, Begoniaceae, Bignoniaceae, Bombacaceae, Bonamia, Brosimum, Brunellia, Brownea, Calliandra, Campomanesia, Cardiospermum, Castilla, Cecropia, Cedrela, Celtis, Copaifera, Coprosma, Cucurbitaceae, Cunoniaceae, Dalbergia, Dioclea, Doliocarpus, Elaeagia, Euterpe, Ficus, Flacourtiaceae, Forsterania, Geonoma, Guapira, Guazuma, Guarea, Hura, Ilex, Inga, Iriartea, Iva xanthifolia-type, Lecythidaceae, Leguminoseae, Licania, Mabea, Macharium, Macrolobium, Macrocarpea, Malpighiaceae, Mauritia, Marcgraviaceae, Maripa, Marattia, Matayba, Melastomatacae, Meliaceae, Miconia, Moraceae, Myrsine, Myrtaceae, Mauritiella, Nyctaginaceae, Ochnaceae, Ocotea-type, Oenocarpus, Oreopanax, Palmae, Panopsis, Parahancornia, Passiflora, Plenckia, Protium, Pseudopanax laetevirens, Rauvolfia, Rhipsalis, Rubiaceae, Sapotaceae, Scheelea, Scleronema, Socratea, Sloanea, Solanaceae, Sophora, Strutanthus, Symphonia, Swartzia, Taperira, Ternstroemia cf. T. brasiliensis, Tetrochiduim, Tetraploa aristata, Thymelaeaceae, Tiliaceae, Trichilia, Trigonia, Vismia, Warsewiczia.

$\mathrm{Te}_{2}$ Abutilon, Acacia, Aegiphila, Alibertia, Apeiba, Apocynaceae, Aspidosperma, Boraginaceae, Bougainvillea, Brosimum Brunellia, Calliandra, Cecropia, Cedrela, Celtis, Combretaceae, Croton, Cucurbitaceae, Dalbergia, Didymopanax, Dioclea, Forsterania, Hippocrateaceae, Humiria, Humulus, Ilex, Iriartea, Leguminoseae, Macrolobium, Mauritia, Melastomataceae, Miconia, Moraceae, Mysine, Myrtaceae, Ochnaceae, Ocotea-type, Palmae, Panopsis, Passiflora, Plenckia, Pseudopanax, Psychotria, Sapotaceae, Scleronema, Serjania, Sophora, Strutanthus, Swartzia, Taperira, Tiliaceae, Vismia, Warsewiczia.

\begin{tabular}{|c|c|}
\hline wtc & Abies, Araucaria, Juniperus, Pinus. \\
\hline $\operatorname{ctc}_{2}$ & $\begin{array}{l}\text { Abies, Araucaria, Araucaria augustifolia, Cupressaceae, Dacrydium, Juniperus, Pilgerodendron, Podocarpus, Prumno- } \\
\text { pitys andina, Saxegothaea conspicua. }\end{array}$ \\
\hline $\operatorname{ctc}_{1}$ & $\begin{array}{l}\text { Austrocedrus chilensis, Cupressaceae, Dacrydium, Fitzroya cupressoides-type, Pilgerodendron, Pinus, Podocarpus, } \\
\text { Prumnopitys andina, Saxegothaea conspicua, Taxodium. }\end{array}$ \\
\hline txts & $\begin{array}{l}\text { Acacia, Aeschynomene, Agave, Anthurium, Aphelandra, Arrabidaea, Atamisquea, Ayenia, Bursera, Byrsonima, Byt- } \\
\text { tneria, Cabomba, Cactaceae, Caryocar, Cayaponia, Cercidium, Chomelia, Chrysophyllum, Chuquiragua, Cissus, Clu- } \\
\text { sia, Combretaceae, Convolvulaceae, Cordia, Cuphea, Curatella, Dodonaea, Echinodorus, Eichhornia, Evolvulus, Hip- } \\
\text { pocrateaceae, Humulus, Hyptis, Ipomoea, Larrea, Lithraea, Malpighiaceae, Manihot, Maprounea, Menispermaceae, } \\
\text { Metopium, Miconia, Mimosa cf M.taimbensis, Palicourea, Peperommia, Phaseolus, Phyllanthus, Polygala, Polylepis- } \\
\text { Acaena, Pouteria, Portulaccaceae undiff., Prosopis, Rhamnaceae, Sapium, Scheffera, Schinus, Sebastiana, Serjania, } \\
\text { Solanaceae, Sloanea, Stryphnodendron, Tecoma, Trixis, Zornia. }\end{array}$ \\
\hline $\mathrm{h}$ & Arenaria, Aristotelia, Asteraceae, Berberidaceae, Berberis, Empetrum, Ericaceae, Sisyrinchium-type. \\
\hline ds & Agave, Atamisquea, Cactaceae, Ephedra, Monttea aphylla. \\
\hline df & Alternanthera, Ephedra, Monttea aphylla, Xyris. \\
\hline
\end{tabular}


Table 4. Continued.

\begin{tabular}{ll}
\hline PFT Codes & Pollen Taxa \\
\hline tf & Acalypha, Acanthaceae, Alchemilla, Alismataceae, Alsophila, Anemia, Antheroceros, Armeria, Artemisia, Assulina, \\
& Asteraceae, Astelia, Begonia, Bernardia, Brassicaceae, Bravaisia, Bromeliaceae, Calyceraceae, Caperonia, Caryophyl- \\
& laceae, Cassia, Cichoriaceae, Cirsium, Cruciferae, Eriogonum, Eriocaulaceae, Euphorbia, Euphorbiaceae, Fabaceae, \\
& Geraniaceae, Gomphorena, Gunnera, Hebenaria, Iresine, Justicia, Lamiaceae, Laportea, Liquidambar, Liliaceae, Lo- \\
& belia, Menispermaceae, Muehlenbeckia, Nertea, Onagraceae, Orchidaceae, Pilea, Polygala, Rhaphithamnus, Rhus, Ru- \\
& biaceae, Smilax, Triumfetta, Umbelliferae, Urticaceae, Verbena, Verbenaceae, Vernonia, Viburnum, Vitis.
\end{tabular}

tef Acalypha, Acanthaceae, Apiaceae, Apium, Artemisia, Astelia, Azara, Borreria, Brassicaceae, Bravaisia, Bromeliaceae, Cassia, Cichoriaceae, Eriocaulaceae, Eriogonum, Euphorbia, Euphorbiaceae, Fabaceae, Genipa, Gordonia, Gunnera, Hippeastrum, Hydrocotyle, Iridaceae, Jungia, Justicia, Lachemella, Lamanonia, Lamiaceae, Laportea, Liliaceae, Lupinus, Malvaceae, Marcgraviaceae, Moritzia-type, Mutisia, Nertea, Onagraceae, Orchidaceae, Pamphalea, Perezia, Phaseolus, Pilea, Piscidia, Plantago, Polemoniaceae, Polygala, Portulaccaceae undiff., Pouteria, Ranunculaceae, Rubiaceae, Rumex, Satureja, Scrophulariaceae, Selaginella, Thalictrum, Triumfetta, Umbelliferae, Urticaceae, Verbenaceae, Vernonia, Vicia, Vitis, Wendtia, Xyris.

sf Alternanthera, Amaranthaceae/Chenopodiaceae, Antheroceros, Armeria, Assulina, Asteraceae, Astelia, Borreria, Calyceraceae, Cardus, Caryophyllaceae, Cardus, Connarus, Cruciferae, Embothrium, Eriogonum, Eryngium, Euphorbia, Euphorbiaceae, Fabaceae, Geraniaceae Gomphorena, Gunnera, Hebenaria, Hippeastrum, Hydrocotyle, Iridaceae, Jungia, Justicia, Liliaceae, Lamiaceae, Liquidambar, Mutisia, Nanodea, Nassauvia-type, Orchidaceae, Oxalis, Phacelia, Physalis, Plantago, Polemoniaceae, Pouteria, Ranunculaceae, Restionaceae, Rosaceae, Rubiaceae, Satureja, Scutellaria-type, Umbelliferae, Urticaceae, Vicia, Vitis, Wendtia, Xyris.

af Arenaria, Astragalus, Azorella, Bartsia-type, Borreria, Bromeliaceae, Bravaisia, Campanulaceae, Cardus, Caryophyllaceae, Deschampsia antarctica, Diphasiastrum complanatum-type, Donatia, Draba, Epilobium, Eriocaulaceae Eriocaulon, Eriogonum, Gaimardia, Gilia, Halenia, Hebenaria, Hippeastrum, Hydrocotyle, Iridaceae, Jamesonia, Labiatae, Lachemella, Lamiaceae, Liquidambar, Lupinus, Lysipomia, Montia, Moritzia-type, Muehlenbeckia, Nassauvia-type, Orchidaceae, Oxalis, Perezia, Plantago, Puya, Quinchamalium, Relbunium, Rosaceae, Rubiaceae, Rumex, Satureja, Scrophulariaceae, Scutellaria-type, Selaginella, Sisyrinchium-type, Umbelliferae, Valeriana, Viola.

\begin{tabular}{|c|c|}
\hline $\mathrm{cp}$ & Apiaceae, Azorella, Gaimardia, Montia, Plantago, Saxifraga. \\
\hline wte & $\begin{array}{l}\text { Aegiphila, Allophylus, Aphelandra, Araliaceae, Azara, Baccharis, Bauhinia, Begoniaceae, Buddleja, Bumelia-type, Clu- } \\
\text { sia, Croton, Daphnopsis, Desfontainia, Elaeocarpaceae, Embothrium, Eucryphia/Caldcluvia paniculata, Euterpe, Fuch- } \\
\text { sia, Geonoma, Geraniaceae, Griselinia, Guettardia, Gunnera, Guttiferae, Hedyosmum, Heliocarpus, Humiria, Labiatae, } \\
\text { Lomatia/Gevuina, Loranthaceae, Ludwigia, Luehea, Malpighiaceae, Matayba, Melastomatacae, Meliaceae, Mimosa, } \\
\text { Mimosa cf. M. scabrella, Mutisia, Myrica, Mysine, Nothofagus obliqua-type, Oreopanax, Palicourea, Proteaceae, } \\
\text { Prunus, Pseudopanax laetevirens, Psychotria, Quercus, Roupala, Sambucus, Solanaceae, Stryphnodendron, Styloceras, } \\
\text { Tepualia stipularis, Tetrochiduim, Thymelaeaceae, Trichilia, Verbenaceae, Viburnum, Warsewiczia, Weinmannia. }\end{array}$ \\
\hline wte $_{1}$ & $\begin{array}{l}\text { Aegiphila, Alnus, Arecaceae, Aragoa, Arcytophyllum, Aristotelia, Azara, Banara, Banisteriopsis, Begoniaceae, Bocco- } \\
\text { nia, Brunellia, Buddleja, Calandrinia, Campanulaceae, Celastraceae, Chuquiraga, Clethra, Daphnopsis, Desfontainia, } \\
\text { Dodonaea, Drimys, Epacridaceae, Ericaceae, Fuchsia, Galium, Gaultheria ulei, Geraniaceae, Gesneriaceae, Hedyos- } \\
\text { mum, Hydrangea, Hypericum, Labiatae, Loranthaceae, Ludwigia, Maytenus, Meliaceae, Meliosma, Muehlenbeckia, } \\
\text { Myrtaceae, Mysine, Nothofagus, Nothofagus antarctica-type, Ostrya-type, Proteaceae, Prunus, Pseudopanax laete- } \\
\text { virens, Quercus, Ribes, Roupala, Sambucus, Solanaceae, Tepualia stipularis, Verbenaceae, Viburnum, Weinmannia. }\end{array}$ \\
\hline wte $_{4}$ & $\begin{array}{l}\text { Abatia, Adesmia, Alchemilla, Alfaroa, Arcytophyllum, Assulina, Asteraceae, Clethra, Colignonia, Dodonaea, Ericaceae, } \\
\text { Gaiadendron, Gaultheria ulei, Guttifera, Laurelia, Muehlenbeckia, Myrteola, Polylepis-Acaena, Ribes, Rosaceae, } \\
\text { Tetrochiduim, Weinmannia. }\end{array}$ \\
\hline ts & $\begin{array}{l}\text { Alnus, Banksia, Carpinus, Cayaponia, Fagus, Fraxinus, Juglans, Loranthaceae, Luehea, Myrica, Populus, Styrax, } \\
\text { Trema, Ulmaceae, Vallea. }\end{array}$ \\
\hline $\mathrm{ts}_{1}$ & $\begin{array}{l}\text { Escallonia, Eugenia, Gordonia, Liquidambar, Luehea, Misodendrum, Myzodendron, Styrax, Ternstroemia cf. } T . \\
\text { brasiliensis, Trema, Vallea. }\end{array}$ \\
\hline aa & $\begin{array}{l}\text { Aragoa, Arcytophyllum, Arenaria, Asteraceae, Baccharis, Cruciferae, Draba, Empetrum, Ephedra, Ericaceae, Erio- } \\
\text { gonum, Escallonia, Gentiana, Gentianaceae, Hypericum, Nassauvia-type, Puya, Rosaceae, Senecio. }\end{array}$ \\
\hline
\end{tabular}




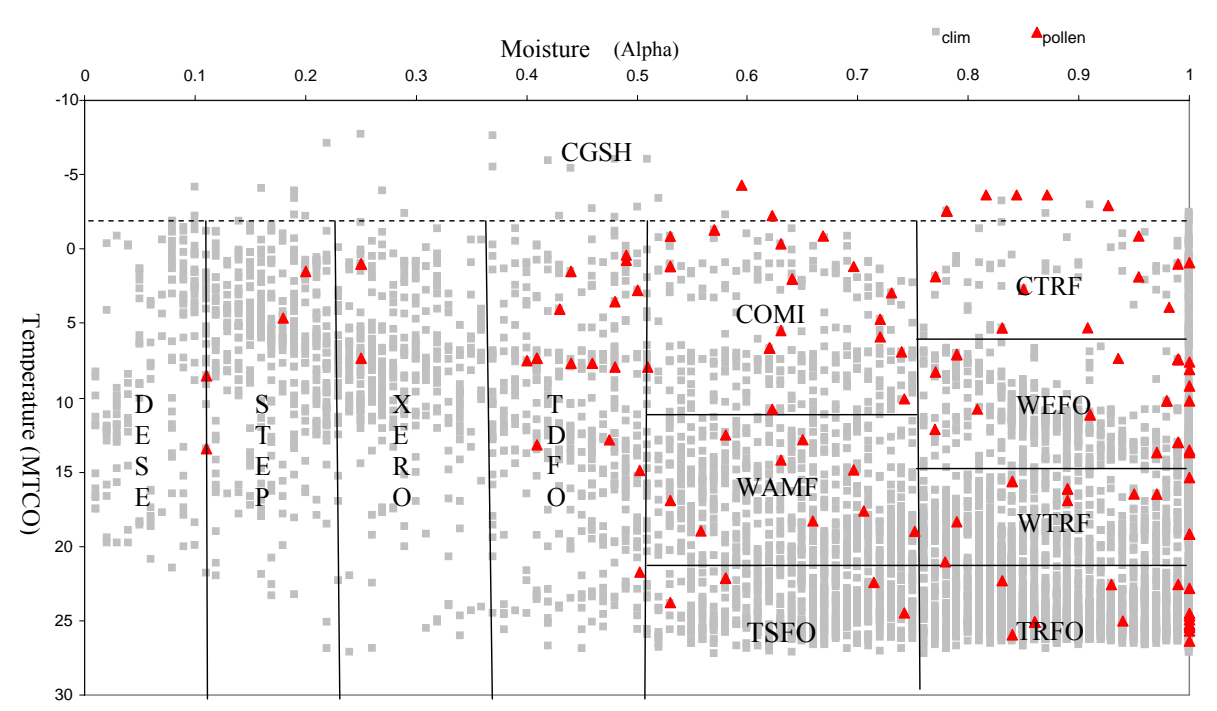

Fig. 5. Theoretical biome scheme for Latin America portrayed on a grid of environmental space along the gradients of temperature (mean temperature of the coldest month) and plant available moisture (alpha). Grey fields reflect the climate space in $100 \times 100 \mathrm{~km}$ grid cells from Mexico to Patagonia as derived from Leemans and Crammer (1991). The location of sites where pollen data is used for the reconstruction of biomes across Latin America are shown as red triangles.

Table 5. Assignment of Latin American plant functional types to Biomes.

\begin{tabular}{|c|c|}
\hline CODES & Plant functional types \\
\hline TRFO & man, tx, $\mathrm{Te}_{1}, \mathrm{Te}_{2}, \mathrm{tf}$ \\
\hline TSFO & $\mathrm{tx}, \mathrm{Tr}_{1}, \mathrm{Tr}_{2}, \mathrm{Te}_{2}, \mathrm{tf}$ \\
\hline TDFO & $\mathrm{Tr}_{2}, \mathrm{tf}, \mathrm{txts}, \mathrm{df}$ \\
\hline WTRF & tx, $\operatorname{Tr}_{1}, \mathrm{Te}_{1}$, wtc, $\mathrm{ctc}_{2}$, tef, wte \\
\hline WEFO & $\mathrm{tx}, \mathrm{Tr}_{2}$, wtc, $\mathrm{ctc}_{2}, \mathrm{ec}$, tef, wte \\
\hline CTRF & tx, h, ctc $_{1}$, tef, wte, wte 1 \\
\hline WAMF & $\mathrm{Tr}_{2}$, wtc, tef, wte, ts \\
\hline COMI & $\mathrm{ctc}_{1}$, tef, wte 1 , wte 4 , ts 1 \\
\hline STEP & sf \\
\hline DESE & $\mathrm{ds}, \mathrm{df}$ \\
\hline CGSH & af, aa, wte $4, h$ \\
\hline CGSS & af, aa, cp \\
\hline
\end{tabular}

vegetation commonly result from the inclusion of high altitude arboreal pollen, this resulting in assignments of cool mixed forest and cool temperate rain forest. The other common assignment is towards steppe; the dominance of the pollen spectra by Poaceae, and lack of shrubby taxa, result in the assignment to the steppe biome. Indeed, the affinity scores to the cool grass/shrubland and steppe biome at most sites, where one of these biomes is dominant, is normally quite close. For the cool mixed forest biome $66 \%$ of sites accurately reconstruct the potential vegetation. The $34 \%$ of "wrong" assignments mainly result in either a reconstruction of cool grass/shrubland, thought to represent possible forest clearance, and the dominance of the vegetation by grassland, or cool temperate rain forest biome due to the numerous shared taxa between these two biomes. $75 \%$ of cool temperate rain forest biome reconstructions match the potential vegetation at the site. The remaining $25 \%$ of the sites mainly show either warm mixed forest or warm temperate rain forest assignments. For the tropical dry forest biome some $90 \%$ of the sites accurately reflect the potential vegetation at the site. For the remaining $10 \%$ of "wrong" assignments the common result is towards a cool temperate rain forest or steppe. For the tropical rain forest biome $85 \%$ of the sites accurately reflect the potential vegetation. For the sites that do not match, a common reconstruction is warm temperate rain forest. This can be explained by the number of Andean elements being present within lowland tropical forests with a couple of sites reconstructing the closely related biome of tropical seasonal forest. This facet of the pollen data is also exemplified by a number $(35 \%)$ of the tropical seasonal forest sites recording the warm temperate rain forest biome. Warm evergreen forest is correctly assigned at $80 \%$ of the sites. Warm temperate rain forest is assigned correctly at $78 \%$ of sites. $75 \%$ of the sites that do not reconstruct this biome "correctly" lead to assignments of tropical rainforest and tropical seasonal forest at low altitudes $(<500 \mathrm{~m})$ and cool mixed forest at high $(>2000 \mathrm{~m})$.

The generally correct biome assignments, in relation to a map of potential vegetation confirm the robustness of our application of the biomisation method to Latin America. Where the match between pollen and potential vegetation reconstructions is relatively low (tropical seasonal forest and warm temperate rain forest), then a common forcing factor, that of "high altitude" plants presently growing at low altitudes 
Table 6. Site locations showing biome changes from the present, $6000 \pm 500$ and $18000 \pm 1000{ }^{14} \mathrm{C}$ yr BP. The relationship between the present observed biome and the biome reconstructed from the modern pollen data is also shown.

\begin{tabular}{|c|c|c|c|c|c|c|c|}
\hline Site & Long. & Latitude & Alt. & Present biome & Modern & 6000 & 18000 \\
\hline Lake Åsa3 & -61.13 & -62.62 & 35 & CGSH & CGSH & & \\
\hline Harberton & -67.16 & -54.88 & 20 & STEP & STEP & STEP & \\
\hline Puerto del Hambre & -70.92 & -53.59 & 5 & CTRF & CTRF & CTRF & \\
\hline La Misión & -67.83 & -53.5 & 5 & STEP & STEP & STEP & \\
\hline Punta Arenas & -70.97 & -53.15 & 75 & CTRF & CTRF & CTRF & CGSH \\
\hline Meseta Latorre 1 & -72.05 & -51.52 & 980 & CGSH & COMI & CTRF & \\
\hline Meseta Latorre 2 & -72.03 & -51.44 & 1000 & CGSH & COMI & CGSH & \\
\hline Torres del Paine & -72.66 & -50.98 & 100 & CTRF & CTRF & TDFO & \\
\hline Moreno Glacier Bog & -73.00 & -50.46 & 200 & CTRF & CTRF & COMI & \\
\hline Patagonia & -72.90 & -50.25 & 50 & CTRF & CTRF & & \\
\hline Patagonia & -72.90 & -50.20 & 20 & CTRF & CTRF & & \\
\hline Patagonia & -72.70 & -50.20 & 80 & CTRF & CTRF & & \\
\hline Patagonia & -72.00 & -50.15 & 150 & CTRF & CTRF & & \\
\hline Patagonia & -71.70 & -50.15 & 180 & CTRF & CTRF & & \\
\hline Patagonia & -71.50 & -50.15 & 180 & CTRF & CTRF & & \\
\hline Patagonia & -71.10 & -50.15 & 180 & CTRF & CTRF & & \\
\hline Patagonia & -69.90 & -50.15 & 180 & CTRF & CTRF & & \\
\hline Patagonia & -69.30 & -50.15 & 180 & CTRF & CTRF & & \\
\hline Patagonia & -71.30 & -50.15 & 190 & CTRF & CTRF & & \\
\hline Patagonia & -70.90 & -50.15 & 190 & CTRF & CTRF & & \\
\hline Patagonia & -70.20 & -50.15 & 190 & CTRF & CTRF & & \\
\hline Patagonia & -69.60 & -50.15 & 190 & CTRF & CTRF & & \\
\hline Patagonia & -72.98 & -50.15 & 20 & CTRF & CTRF & & \\
\hline Patagonia & -70.50 & -50.15 & 200 & CTRF & CTRF & & \\
\hline Patagonia & -72.95 & -50.15 & 50 & CTRF & CTRF & & \\
\hline Patagonia & -72.90 & -50.15 & 50 & CTRF & CTRF & & \\
\hline Patagonia & -72.85 & -50.15 & 60 & CTRF & CTRF & & \\
\hline Patagonia & -72.75 & -50.15 & 60 & CTRF & CTRF & & \\
\hline Patagonia & -72.80 & -50.15 & 70 & CTRF & CTRF & & \\
\hline Patagonia & -72.55 & -50.10 & 100 & CTRF & CTRF & & \\
\hline Patagonia & -71.95 & -50.10 & 180 & CTRF & CTRF & & \\
\hline Patagonia & -69.00 & -50.10 & 190 & CTRF & CTRF & & \\
\hline Patagonia & -68.90 & -50.05 & 100 & CTRF & CTRF & & \\
\hline Patagonia & -72.00 & -50.05 & 150 & CTRF & CTRF & & \\
\hline Patagonia & -72.90 & -50.05 & 50 & CTRF & CTRF & & \\
\hline Patagonia & -68.60 & -50.05 & 50 & CTRF & CTRF & & \\
\hline Patagonia & -68.10 & -50.00 & 0 & CTRF & CTRF & & \\
\hline Patagonia & -68.30 & -50.00 & 20 & CTRF & CTRF & & \\
\hline Dichan & -73.88 & -49.66 & 50 & CTRF & CTRF & CTRF & \\
\hline Puerto Eden & -74.41 & -49.13 & 50 & CTRF & CTRF & CTRF & \\
\hline La Esperanza & -72.83 & -46.63 & 330 & CTRF & CTRF & & \\
\hline LagoBsAs & -71.45 & -46.44 & 230 & CTRF & WAMF & & \\
\hline Laguna Six Minutes & -74.33 & -46.43 & 50 & CTRF & CTRF & CTRF & CTRF \\
\hline Laguna Stibnite & -74.43 & -46.43 & 50 & CTRF & CTRF & CGSH & \\
\hline Laguna Stibnite & -74.38 & -46.43 & 50 & CTRF & CTRF & CTRF & $\mathrm{CGSH}$ \\
\hline Pico Salam & -67.43 & -45.42 & 637 & STEP & STEP & & \\
\hline Laguna Lincoln & -74.07 & -45.34 & 50 & CTRF & CTRF & CTRF & $\mathrm{CGSH}$ \\
\hline Laguna Lofel & -74.43 & -44.85 & 50 & CTRF & CTRF & CTRF & $\mathrm{CGSH}$ \\
\hline Estero Huitanque & -73.82 & -43.61 & 52 & CTRF & CTRF & COMI & \\
\hline AustroEsqu & -71.47 & -42.66 & 1100 & CTRF & CTRF & & \\
\hline Mayol & -73.75 & -42.64 & 75 & CTRF & CTRF & CTRF & \\
\hline Puchilco & -73.62 & -42.63 & 110 & CTRF & CTRF & CTRF & \\
\hline AlercesNor & -71.60 & -42.56 & 800 & CTRF & CGSH & & \\
\hline San Pedro & -73.95 & -42.25 & 650 & CTRF & CTRF & & \\
\hline Chepu & -73.66 & -42.17 & 140 & CTRF & CTRF & & \\
\hline
\end{tabular}


Table 6. Continued.

\begin{tabular}{|c|c|c|c|c|c|c|c|}
\hline Site & Long. & Latitude & Alt. & Present biome & Modern & 6000 & 18000 \\
\hline Mallin Book & -71.58 & -41.33 & 800 & CTRF & COMI & CTRF & \\
\hline Comallo & -70.21 & -41.01 & 815 & CGSH & STEP & & \\
\hline Puerto Octay PM13 & -72.90 & -40.93 & 120 & CTRF & CTRF & CTRF & COMI \\
\hline Espuma & -63.25 & -40.67 & 50 & TDFO & TDFO & & \\
\hline Primavera & -71.18 & -40.66 & 800 & CTRF & CGSH & TDFO & \\
\hline Encantado & -71.13 & -40.66 & 960 & CTRF & COMI & & \\
\hline Ruta 3.4 & -62.79 & -40.50 & 20 & STEP & STEP & & \\
\hline Ruta 3.3 & -62.59 & -40.08 & 20 & STEP & STEP & & \\
\hline Caunahue & -72.00 & -40.00 & 500 & CTRF & & CTRF & \\
\hline Ruta 250.19 & -65.58 & -39.54 & 117 & STEP & STEP & & \\
\hline Pedro Luro & -62.53 & -39.50 & 20 & STEP & STEP & & \\
\hline Origone & -62.43 & -39.08 & 20 & STEP & STEP & & \\
\hline Gaviotas & -63.65 & -39.07 & 90 & TDFO & TDFO & & \\
\hline Salina Anzotegui & -63.77 & -39.06 & -5 & STEP & STEP & STEP & \\
\hline Cueva Haichol & -70.66 & -38.58 & 1050 & STEP & STEP & STEP & \\
\hline Arroyo Sauce Chico & -62.23 & -38.07 & 85 & STEP & STEP & & \\
\hline Cerro La China & -58.64 & -37.84 & 200 & STEP & TDFO & TDFO & \\
\hline Empalme Querandíes & -60.65 & -37.00 & 105 & STEP & STEP & TDFO & \\
\hline Veranada Pelan & -70.38 & -36.88 & 1860 & CGSH & CGSH & TDFO & \\
\hline Vaca Lauquen & -71.08 & -36.83 & 1450 & CTRF & COMI & CGSH & \\
\hline Veranada Vulkanpickel & -70.41 & -36.68 & 2800 & CGSH & STEP & TDFO & \\
\hline Salado & -69.75 & -35.33 & 3200 & CGSH & CGSH & & \\
\hline Salina 2 & -69.33 & -32.25 & 2000 & CGSH & STEP & STEP & \\
\hline Serra do Rio Rastro & -49.55 & -28.55 & 1420 & WTRF & WTRF & CGSH & \\
\hline Morro da Igreja & -49.86 & -28.18 & 1800 & WTRF & WTRF & WTRF & \\
\hline Serra da Boa Vista & -49.15 & -27.70 & 1160 & WTRF & WTRF & CTRF & \\
\hline Rano Kao & -109.43 & -27.18 & 110 & TDFO & TDFO & & \\
\hline Rano Raraku Bore 3 & -109.28 & -27.16 & 75 & TDFO & TDFO & TDFO & TDFO \\
\hline Rano Aroui & -109.40 & -27.08 & 425 & TDFO & TDFO & CGSH & WTRF \\
\hline Poço Grande & -48.86 & -26.41 & 10 & WTRF & WTRF & & \\
\hline Reserva Volta Velha & -48.38 & -26.04 & 0 & WTRF & WTRF & & \\
\hline Atlantic & -48.35 & -25.95 & 200 & WTRF & WTRF & & \\
\hline Colombo & -49.23 & -25.33 & 920 & TSFO & TSFO & & \\
\hline Serra Campos Gerais & -50.21 & -24.66 & 1200 & WTRF & WAMF & TDFO & \\
\hline Aguilar & -65.75 & -23.83 & 4000 & CGSH & CTRF & STEP & \\
\hline Rio da Curuá & -48.83 & -23.83 & 800 & WTRF & & TRFO & \\
\hline Tumbre 2 & -67.78 & -23.31 & 3880 & CGSH & CGSH & STEP & \\
\hline Aguas Calientas & -67.42 & -23.08 & 4210 & CGSH & CGSH & CGSH & \\
\hline Botucatu & -48.00 & -23.00 & 700 & WTRF & WTRF & & \\
\hline Curcuab & -48.00 & -23.00 & 700 & WTRF & WTRF & & \\
\hline Lagoa da Caço & -43.43 & -22.97 & 5 & TDFO & & TDFO & STEP \\
\hline Morro de Itapeva & -45.63 & -22.78 & 1850 & WTRF & WEFO & TDFO & $\mathrm{CGSH}$ \\
\hline Assis & -50.50 & -22.68 & 540 & TSFO & TSFO & & \\
\hline Lagoa Santa & -47.45 & -22.36 & 630 & TDFO & TDFO & & \\
\hline Bauru & -49.07 & -22.32 & 570 & TSFO & TSFO & & \\
\hline Brotas & -48.08 & -22.29 & 700 & WTRF & WTRF & & \\
\hline Salitre & -46.78 & -19.00 & 1050 & WTRF & WTRF & TDFO & $\mathrm{CGSH}$ \\
\hline Ajata & -69.20 & -18.25 & 4700 & CGSH & CGSH & & \\
\hline Sajama & -68.88 & -18.16 & 4250 & CGSH & CGSH & & \\
\hline Lago do Pires & -42.21 & -17.95 & 390 & TSFO & TSFO & TSFO & \\
\hline Wasa Mayu & -65.91 & -17.54 & 2720 & COMI & & CGSH & STEP \\
\hline Crominia & -49.45 & -17.28 & 200 & TDFO & TDFO & TSFO & TDFO \\
\hline Mt. Blanco & -67.35 & -17.02 & 4780 & CGSS & & CGSS & \\
\hline Lake Huinãmimarca & -69.00 & -16.50 & 3765 & CGSH & CGSH & CGSH & CGSS \\
\hline Cerro Calvario & -68.50 & -16.50 & 3950 & CGSH & $\mathrm{CGSH}$ & CGSH & \\
\hline
\end{tabular}


Table 6. Continued.

\begin{tabular}{|c|c|c|c|c|c|c|c|}
\hline Site & Long. & Latitude & Alt. & Present biome & Modern & 6000 & 18000 \\
\hline Rio Kaluyo & -68.13 & -16.43 & 4070 & CGSH & CGSH & STEP & \\
\hline Chacaltaya 1 & -68.13 & -16.36 & 4750 & CGSH & CGSH & CGSH & \\
\hline Cumre Unduavi & -68.03 & -16.33 & 4620 & CGSS & CGSS & CGSS & \\
\hline Rio Jequitinhonha & -38.92 & -15.85 & 80 & TDFO & TDFO & & \\
\hline Brasilia 1 & -47.66 & -15.59 & 1030 & TDFO & CTRF & & \\
\hline Aguads Emendadas & -47.58 & -15.56 & 200 & TDFO & CTRF & TSFO & $\mathrm{CGSH}$ \\
\hline Cuiaba & -55.86 & -15.35 & 350 & TDFO & TDFO & & \\
\hline Amarete & -68.98 & -15.23 & 4000 & CGSH & CGSH & CGSH & \\
\hline Cotapampa & -69.11 & -15.21 & 4450 & CGSH & CGSH & CGSH & \\
\hline Katantica & -69.18 & -14.8 & 4820 & CGSH & CGSH & CGSH & \\
\hline Laguna Chaplin & -61.05 & -14.50 & 750 & TSFO & TSFO & TDFO & TSFO \\
\hline Rio de Contas & -39.00 & -14.28 & 80 & TDFO & STEP & & \\
\hline Laguna Bella Vista & -61.56 & -13.58 & 750 & TSFO & TSFO & TSFO & TDFO \\
\hline Laguna Jeronimo & -75.21 & -11.78 & 4450 & CGSH & CGSH & CGSH & \\
\hline Laguna Pomacocha & -75.50 & -11.75 & 4450 & CGSH & CGSH & CGSH & \\
\hline Laguna Paca & -75.50 & -11.71 & 3600 & CGSH & CTRF & & \\
\hline Laguna Tuctua & -75.00 & -11.66 & 4250 & CGSH & COMI & WTRF & \\
\hline Laguna Milloc & -76.35 & -11.56 & 4325 & CGSH & CGSH & CGSH & \\
\hline Laguna Junin2 & -76.18 & -11.00 & 4100 & CGSH & COMI & COMI & CGSH \\
\hline Laguna Huatacocha & -76.55 & -10.76 & 4500 & CGSH & CGSH & CGSH & \\
\hline Rio São Francisco & -43.00 & -10.46 & 400 & TDFO & TDFO & TSFO & \\
\hline Saquinho & -43.23 & -10.44 & 480 & TSFO & TSFO & WEFO & \\
\hline Rio São Francisco & -36.50 & -10.26 & 80 & TDFO & STEP & & \\
\hline Katira & -63.00 & -9.00 & 750 & TDFO & TDFO & TDFO & TDFO \\
\hline Campina Grande I & -35.75 & -7.23 & 70 & TSFO & STEP & & \\
\hline Lagoa Grande & -47.45 & -7.08 & 75 & TDFO & CTRF & & \\
\hline Picos & -41.40 & -7.06 & 70 & TDFO & WTRF & & \\
\hline Lago Bolim & -35.18 & -6.04 & 90 & TDFO & STEP & & \\
\hline Rio Protengi & -35.25 & -5.78 & 80 & TDFO & CTRF & & \\
\hline Mirim & -35.30 & -5.68 & 70 & TDFO & STEP & & \\
\hline Rio Mirim & -35.40 & -5.64 & 70 & TDFO & WTRF & & \\
\hline Rio (unclear) & -38.00 & -5.50 & 50 & TDFO & CTRF & & \\
\hline Carajas & -48.00 & -5.00 & 150 & TSFO & TSFO & TSFO & TSFO \\
\hline Ciudad Universitaria X & -74.18 & -4.75 & 2560 & COMI & COMI & WAMF & WAMF \\
\hline Rio Jaguaribe II & -37.76 & -4.55 & 50 & TDFO & TDFO & & \\
\hline Rio Jaguaribe I & -37.75 & -4.43 & 50 & TDFO & STEP & & \\
\hline Lake Surucucho & -78.95 & -3.75 & 970 & WTRF & CTRF & WTRF & \\
\hline Ayauch & -78.13 & -2.09 & 550 & WTRF & WTRF & TSFO & \\
\hline Llaviucu & -79.43 & -1.83 & 3120 & CTRF & WTRF & & \\
\hline Indanza & -78.83 & -1.53 & 2100 & CTRF & WTRF & & \\
\hline Kumpack & -78.51 & -1.53 & 700 & WTRF & WTRF & & \\
\hline Puyo Bog & -79.06 & -1.43 & 953 & WTRF & WTRF & & \\
\hline Rum Tum & -79.03 & -1.13 & 2392 & CTRF & WTRF & & \\
\hline Yambo & -79.03 & -1.03 & 2600 & CTRF & WTRF & & \\
\hline Lago Crispim & -48.00 & -0.8 & 0 & TRFO & STEP & TSFO & \\
\hline Lagoa da Curuça2 & -47.85 & -0.76 & 35 & TRFO & TRFO & TRFO & \\
\hline Mariñame-II & -72.03 & -0.66 & 160 & TRFO & TRFO & WTRF & \\
\hline Monica-1 & -72.50 & -0.60 & 160 & TRFO & TRFO & TRFO & \\
\hline Añangucocha & -77.03 & -0.53 & 280 & TRFO & WTRF & & \\
\hline Limoncocha & -76.66 & -0.38 & 230 & TRFO & CTRF & & \\
\hline Cayambe & -78.03 & -0.03 & 4350 & CGSH & CGSH & CGSH & \\
\hline Lago Agrio & -77.03 & 0.03 & 330 & TRFO & WTRF & & \\
\hline San Marcos & -79.03 & 0.03 & 3400 & CGSH & TDFO & & \\
\hline Santa Cecilia & -77.03 & 0.04 & 330 & TRFO & WTRF & & \\
\hline Lake Santa Cecilia & -77.02 & 0.06 & 330 & TRFO & TRFO & & \\
\hline Cuyabeno & -77.01 & 0.08 & 280 & TRFO & WTRF & & \\
\hline
\end{tabular}


Table 6. Continued.

\begin{tabular}{|c|c|c|c|c|c|c|c|}
\hline Site & Long. & Latitude & Alt. & Present biome & Modern & 6000 & 18000 \\
\hline Cunro & -79.03 & 0.08 & 2800 & CTRF & WTRF & & \\
\hline Mera & -76.92 & 0.11 & 1100 & WTRF & WTRF & & \\
\hline Lake Agrio & -76.92 & 0.11 & 330 & TRFO & TRFO & & \\
\hline Yaguara cocha & -79.03 & 0.13 & 2210 & CTRF & WTRF & & \\
\hline La Pata & -66.66 & 0.25 & 300 & TRFO & TSFO & WTRF & WTRF \\
\hline Lagoa das Patas & -66.68 & 0.26 & 300 & TRFO & WTRF & TSFO & WTRF \\
\hline Piusbi & -77.89 & 1.66 & 200 & TRFO & TRFO & TRFO & \\
\hline Pitalito & -76.50 & 1.75 & 1300 & WEFO & WEFO & TSFO & \\
\hline Piagua & -76.50 & 2.30 & 1700 & WEFO & WEFO & WEFO & \\
\hline Pantano de Genagra & -76.50 & 2.50 & 1750 & WEFO & TDFO & WEFO & \\
\hline Rio Timbio & -76.50 & 2.50 & 1750 & WEFO & WEFO & WEFO & \\
\hline El Camito & -76.60 & 2.53 & 50 & TRFO & TRFO & & \\
\hline Loma Linda & -73.35 & 3.22 & 310 & TDFO & TDFO & TDFO & \\
\hline Lago Agua Sucia & -73.54 & 3.46 & 260 & TDFO & TDFO & STEP & \\
\hline El Gobernador & -75.00 & 3.95 & 3815 & CTRF & CTRF & WTRF & \\
\hline La Guitarra & -74.28 & 4.00 & 3450 & COMI & COMI & CTRF & \\
\hline La Primavera & -74.13 & 4.00 & 3525 & CGSH & CGSH & COMI & \\
\hline Corazón Partido & -74.25 & 4.00 & 4100 & CGSH & CGSH & & \\
\hline El Trinagulo & -74.25 & 4.00 & 4100 & CGSH & CGSH & & \\
\hline Carimagua & -74.14 & 4.04 & 180 & TDFO & TDFO & CGSS & \\
\hline La Rabona & -74.25 & 4.05 & 4000 & CGSH & CGSH & CTRF & \\
\hline El Piñal & -70.40 & 4.09 & 185 & TDFO & TDFO & TDFO & TDFO \\
\hline Alsacia & -74.11 & 4.09 & 3100 & COMI & WAMF & COMI & \\
\hline Andabobos & -74.15 & 4.09 & 3570 & CGSH & CGSH & CGSH & \\
\hline Ubaqué & -73.55 & 4.33 & 2000 & WEFO & WEFO & & \\
\hline De la América & -74.00 & 4.33 & 3550 & CGSH & $\mathrm{CGSH}$ & CTRF & \\
\hline Turbera de Calostros & -73.48 & 4.41 & 3730 & CGSH & CGSH & & \\
\hline Laguna Angel & -70.54 & 4.45 & 205 & TDFO & TSFO & TDFO & \\
\hline Libano & -75.50 & 4.50 & 1820 & WEFO & WEFO & COMI & \\
\hline de Pedro Palo III & -74.41 & 4.50 & 2000 & COMI & WTRF & WTRF & \\
\hline Paramo Palacio & -73.88 & 4.76 & 3550 & CGSH & CGSH & CGSH & \\
\hline Greja & -73.70 & 4.86 & 4000 & CGSH & CGSH & CGSH & \\
\hline Sardinas & -69.45 & 4.95 & 180 & TDFO & TDFO & TDFO & \\
\hline Herrera & -73.91 & 5.00 & 2000 & COMI & CGSH & COMI & CTRF \\
\hline Agua Blanca & -74.45 & 5.0 & 3250 & COMI & COMI & CTRF & $\mathrm{CGSH}$ \\
\hline El Abra II & -73.96 & 5.02 & 2570 & COMI & CTRF & CTRF & \\
\hline Paramo de Peña Negra & -74.09 & 5.09 & 3625 & CGSH & CGSH & COMI & \\
\hline ODP site 932 & -47.03 & 5.18 & 0 & TRFO & WTRF & WTRF & TSFO \\
\hline Comprida & -47.63 & 5.18 & 130 & WTRF & WTRF & TRFO & \\
\hline Geral & -47.53 & 5.18 & 130 & WTRF & WTRF & TRFO & \\
\hline Paramo de Laguna Verde & -74.00 & 5.25 & 3625 & CGSH & CGSH & CGSH & \\
\hline Fúquene II & -73.87 & 5.50 & 2580 & COMI & CGSH & CTRF & CTRF \\
\hline Jotaordo & -76.66 & 5.66 & 0 & TRFO & TRFO & & \\
\hline Cienaga del Visitador & -72.83 & 6.13 & 3300 & COMI & COMI & CGSH & \\
\hline Bobos & -72.85 & 6.13 & 3800 & CGSH & CGSH & & \\
\hline Ciega I & -72.31 & 6.50 & 3510 & COMI & CTRF & & \\
\hline Valle de Lagunillas & -72.34 & 6.50 & 3880 & CGSH & CGSH & CTRF & \\
\hline Cana, Darien & -77.58 & 7.68 & 1000 & TSFO & TSFO & & \\
\hline Cana Swamp & -77.59 & 7.74 & 500 & TRFO & TRFO & & \\
\hline Wodehouse Swamp & -77.58 & 7.75 & 500 & TRFO & TRFO & & \\
\hline La Chonta & -82.00 & 8.00 & 2310 & CTRF & CTRF & COMI & WAMF \\
\hline El Valle & -79.78 & 8.43 & 500 & TSFO & TSFO & & TDFO \\
\hline La Yeguada, & -80.78 & 8.43 & 650 & TSFO & TSFO & TSFO & \\
\hline Cerro Campana & -79.93 & 8.63 & 800 & WTRF & WTRF & & \\
\hline Quebrada Nelson & -82.31 & 8.66 & 1130 & WTRF & WTRF & & \\
\hline Laguna Volcán & -82.75 & 8.75 & 1500 & WTRF & WAMF & & \\
\hline
\end{tabular}


Table 6. Continued.

\begin{tabular}{|c|c|c|c|c|c|c|c|}
\hline Site & Long. & Latitude & Alt. & Present biome & Modern & 6000 & 18000 \\
\hline Valle Laguna Negra & -70.76 & 8.79 & 3450 & CGSH & CGSH & & \\
\hline Valle Laguna Victoria & -70.79 & 8.80 & 3250 & CGSH & CGSH & $\mathrm{CGSH}$ & \\
\hline Horsefly Ridge & -82.24 & 8.83 & 1150 & WTRF & WTRF & & \\
\hline Volcan Baru & -82.52 & 8.85 & 2600 & CTRF & CTRF & & \\
\hline El Bosque & -75.45 & 8.85 & 3650 & CGSH & CGSH & & \\
\hline Finca Lerida & -82.45 & 8.87 & 1630 & WTRF & WAMF & & \\
\hline Volcan Irazu & -82.52 & 8.88 & 2300 & CTRF & CTRF & & \\
\hline Paramo de Miranda & -70.85 & 8.91 & 3290 & CGSH & CGSH & CGSH & \\
\hline Panama & -80.87 & 9.00 & 100 & TSFO & WTRF & TSFO & \\
\hline Ocelot Pond & -79.59 & 9.12 & 20 & TSFO & WTRF & & \\
\hline Soberania & -79.66 & 9.13 & 20 & TSFO & WTRF & & \\
\hline Paramo Piedras Blancas & -70.83 & 9.16 & 4080 & CGSH & CGSH & CGSH & \\
\hline Pipeline Rd & -79.66 & 9.33 & 40 & TSFO & WTRF & & \\
\hline Barro Colorado Island & -79.75 & 9.35 & 50 & TSFO & WTRF & & \\
\hline Lago Chirripó & -83.48 & 9.48 & 3520 & CTRF & WAMF & & \\
\hline Talamancas & -83.72 & 9.5 & 2500 & CTRF & WAMF & & \\
\hline Lago de las Morrenas & -83.49 & 9.50 & 3480 & CTRF & WAMF & CTRF & \\
\hline Bog 70 & -83.85 & 9.61 & 2670 & CTRF & WTRF & & \\
\hline Tres de Junio & -83.87 & 9.62 & 2670 & CTRF & CTRF & & \\
\hline Bog 68 & -83.85 & 9.64 & 2670 & CTRF & WTRF & & \\
\hline Asuncion & -83.75 & 9.64 & 3340 & CTRF & CTRF & & \\
\hline Carara Biological Reserve & -84.62 & 9.73 & 0 & TSFO & TSFO & & \\
\hline Quebrador & -83.84 & 9.74 & 3040 & CTRF & CTRF & & \\
\hline Cataracta, Carara 1 & -84.63 & 9.83 & 270 & TSFO & WTRF & & \\
\hline Cataracta, Carara 2 & -84.63 & 9.85 & 270 & TSFO & WTRF & & \\
\hline Carara & -84.60 & 9.88 & 35 & WTRF & WTRF & & \\
\hline Laguna Bonilla & -83.61 & 9.99 & 380 & TSFO & WTRF & & \\
\hline Laguna Barva & -84.11 & 10.14 & 2840 & CTRF & WTRF & & \\
\hline Laguna Botos & -84.18 & 10.18 & 2600 & CTRF & WTRF & & \\
\hline Laguna Palmita & -84.95 & 10.18 & 60 & TSFO & WTRF & & \\
\hline Bosque Alegre & -84.21 & 10.21 & 740 & WTRF & WTRF & & \\
\hline Laguna González & -84.45 & 10.25 & 710 & WTRF & WTRF & & \\
\hline Laguna Congo & -84.29 & 10.27 & 740 & WTRF & WTRF & & \\
\hline Laguna Hule & -84.19 & 10.27 & 740 & WTRF & WTRF & & \\
\hline Laguna María Aguilar & -84.18 & 10.27 & 770 & WTRF & WTRF & & \\
\hline Monteverde, Heredia & -84.8 & 10.3 & 1500 & WTRF & WTRF & & \\
\hline Volcan Poas & -84.19 & 10.3 & 2580 & CTRF & CTRF & & \\
\hline Brauillo Carillo, Heredia & -83.94 & 10.3 & 630 & WTRF & WTRF & & \\
\hline Lake Valencia & -67.75 & 10.32 & 403 & STEP & STEP & TDFO & \\
\hline Laguna Río Cuarto & -84.18 & 10.34 & 380 & WTRF & WTRF & & \\
\hline La Selva, Heredia & -84 & 10.43 & 80 & TSFO & TSFO & & \\
\hline La Pacifica, Guanacaste & -85.11 & 10.45 & 110 & TSFO & TSFO & & \\
\hline Cantarrana Swamp & -84.00 & 10.45 & 36 & TSFO & WTRF & & \\
\hline Sendro Sedro Swamp & -84.00 & 10.46 & 40 & TSFO & WTRF & & \\
\hline Laguna La Palma & -84.73 & 10.49 & 570 & WTRF & WTRF & & \\
\hline Laguna Cedeño & -84.71 & 10.49 & 610 & WTRF & WTRF & & \\
\hline Tortuguero & -83.53 & 10.53 & 0 & TSFO & WTRF & & \\
\hline Santa Rosa2 & -85.64 & 10.83 & 0 & TSFO & TSFO & & \\
\hline Santa Rosa1 & -85.66 & 10.84 & 0 & TRFO & TRFO & & \\
\hline Boca de Lopez & -75.36 & 10.85 & 0 & TRFO & TRFO & & \\
\hline Cafetal, Guanacaste & -85.65 & 10.85 & 300 & TSFO & WTRF & & \\
\hline Santa Rosa3 & -85.62 & 10.86 & 280 & TSFO & TSFO & & \\
\hline Santa Rosa4 & -85.62 & 10.86 & 280 & TSFO & WTRF & & \\
\hline Escondido & -85.61 & 10.87 & 280 & TSFO & WTRF & & \\
\hline Volcán Cacao & -85.47 & 10.92 & 1000 & WTRF & WTRF & & \\
\hline Sierra de Cuchumatanes5 & -91.00 & 15.75 & 2800 & WAMF & WAMF & & \\
\hline
\end{tabular}


Table 6. Continued.

\begin{tabular}{lrrrrrrr}
\hline Site & Long. & Latitude & Alt. & Present biome & Modern & 6000 & 18000 \\
\hline Sierra de Cuchumatanes4 & -91.25 & 15.75 & 3000 & CTRF & CTRF & & \\
Sierra de Cuchumatanes3 & -91.5 & 15.75 & 3400 & CTRF & CTRF & & \\
Sierra de Cuchumatanes2 & -91.75 & 15.75 & 3600 & WAMF & WAMF & & \\
Sierra de Cuchumatanes1 & -92.00 & 15.75 & 4200 & CGSH & CTRF & & \\
Lago Quexil & -89.88 & 16.92 & 110 & TSFO & WAMF & WEFO & WAMF \\
Lake Peten-Itza & -90.00 & 17.25 & 200 & TDFO & TDFO & WEFO & \\
Lago Catemaco & -95.00 & 18.66 & 340 & TRFO & WAMF & & \\
Zempoala & -99.30 & 19.20 & 3100 & WAMF & WAMF & & \\
Quila & -99.20 & 19.30 & 2800 & WAMF & WAMF & WAMF & \\
Lake Texcoco & -99.12 & 19.44 & 2330 & WAMF & WAMF & WAMF & WAMF \\
Chalco Lake & -99.00 & 19.50 & 2240 & WAMF & WAMF & WAMF & WAMF \\
Lake Pátzcuaro & -101.58 & 19.58 & 2044 & WAMF & WAMF & WAMF & WAMF \\
San Jose Chulchaca & -90.13 & 20.86 & 1 & TDFO & TDFO & WEFO & \\
Lake Coba & -87.55 & 20.86 & 100 & WAMF & WAMF & WEFO & \\
\hline
\end{tabular}

appears important. For other sites where the reconstructed biome does not match the potential vegetation map a series of different explanations, particularly local site-specific factors such as human impact, can be invoked, these will be discussed fully. Taking our modern pollen to potential vegetation calibration, and the design of the matrices that drive it, we reconstruct vegetation at past time intervals with "cautious confidence".

\section{$3.26000{ }^{14} \mathrm{C}$ yr BP biome reconstruction}

The biomes reconstructed at $6000 \pm 500{ }^{14} \mathrm{C}$ yr BP (Fig. 8) show relatively small patterns of change compared to the present. $49 \%$ of the sites retain the same biome assignment as present (Table 6). In southeastern Brazil, the majority of the sites that were previously assigned to the warm temperate forest biome remain unchanged. A number of sites (e.g. Serra Campos Gerais, Rio São Francisco, Aguads Emendadas) record tropical dry forest at $6000 \pm 500{ }^{14} \mathrm{C} \mathrm{yr}$ BP replacing tropical seasonal forest (Lagiuna Angel, Laguna Chaplin) record at the present. Sites assigned to tropical rain forest and tropical seasonal forest today mainly remain unchanged at $6000 \pm 500{ }^{14} \mathrm{C}$ yr BP. Steppe continues to be reconstructed in southeastern Argentina, as today. However, a number of sites had substantially more arboreal components at $6000 \pm 500{ }^{14} \mathrm{C}$ yr BP than today, for example Empalme Querandíes and Lake Valencia show a transition from steppe to tropical dry forest. An expansion of steppe is recorded at sites previously assigned to cold mixed forest on the Cordillera de los Andes. Unlike sites in southernmost South America that similarly record steppe, these sites also contain significant amounts of Alnus and Podocarpus indicative of "parkland" at this time. On closer inspection of the affinity scores, sites record an increased affinity to cool temperate rain forest, primarily due to increased amounts of Nothofagus pollen, although this was not sufficiently numerous to produce a cool temperate rain forest assignment. Southernmost South America continues to have a mixture of cool mixed forest, cool grass/shrubland, steppe and cool temperate rain forest biomes, the latter being dominant. Along the southern Andean spine, the assignments do not differ greatly from the modern assignment. There are broadly similar assignments to the present at Colombian sites although there is a slight increase in the number of cool mixed forest and cool temperate rain forest biome assignments relative to cool grass/shrubland of the present day. The sites where this occurs (e.g. Primevera, and Páramo de Peña Negra) are located at high altitude and may reflect either an extension of the forest line or increased distribution of Andean forest that predates early human impact. Sites in coastal northern South America show a transition to tropical dry forest and tropical seasonal forest from steppe and tropical dry forest respectively, both indicative of a relatively mesic environment. A number of sites on the Yucatán peninsula show a clear distribution of warm evergreen forest at $6000 \pm 500{ }^{14} \mathrm{C}$ yr BP changing from the warm mixed forest and tropical dry forest reconstruction for the present day. These transitions are not recorded everywhere, for example sites located in the Mexican highlands retain the same biome assignment at the present - warm mixed forest.

\section{$3.318000{ }^{14} \mathrm{C}$ yr BP biome reconstruction}

Vegetation at $18000 \pm 1000{ }^{14} \mathrm{C}$ yr BP was substantially different from the present-day, or that reconstructed at $6000 \pm 500{ }^{14} \mathrm{C}$ yr BP (Fig. 9). The intensity of this vegetation transformation is demonstrated by $74 \%$ of the sites change the biome assignment relative to the two previous periods. In Amazonia, tropical seasonal forest and tropical dry forest is recorded instead of tropical rain forest 

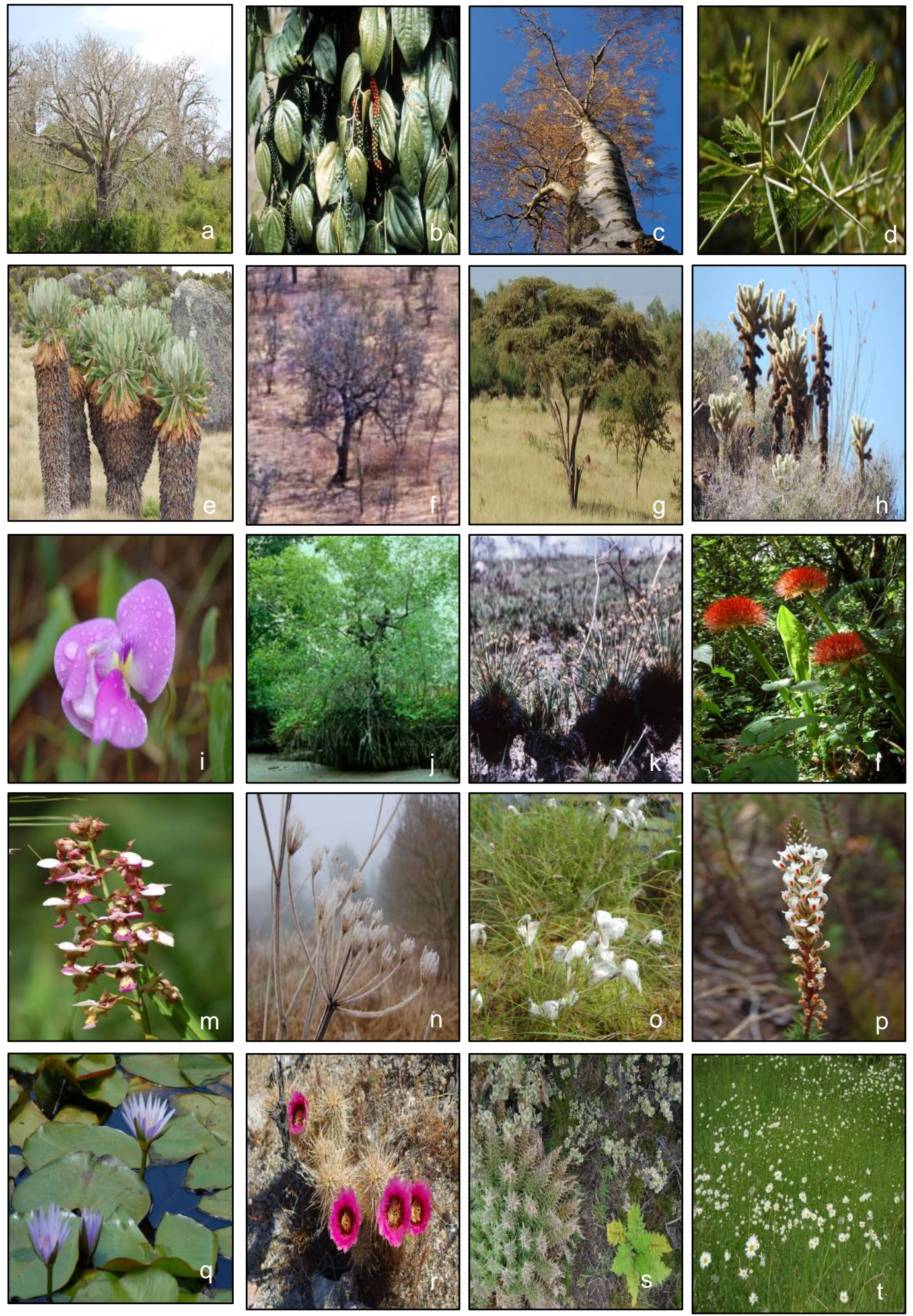

Fig. 6. Latin America plant functional types: (tropical rain green tree (a), tropical evergreen tree (b), temperate summer-green tree (c), tropical xerophytic tree/shrub (d), arctic shrub (e), desert shrub (f), dry tropical rain green tree (h), desert shrub (i), tropical forb (i), mangrove (j), xerophytic forb (k), tropical forb (l), temperate forb $(\mathbf{m}, \mathbf{n})$, grass (o), alpine forb (p), aquatic (q), desert forb (r, s) grass (t). 


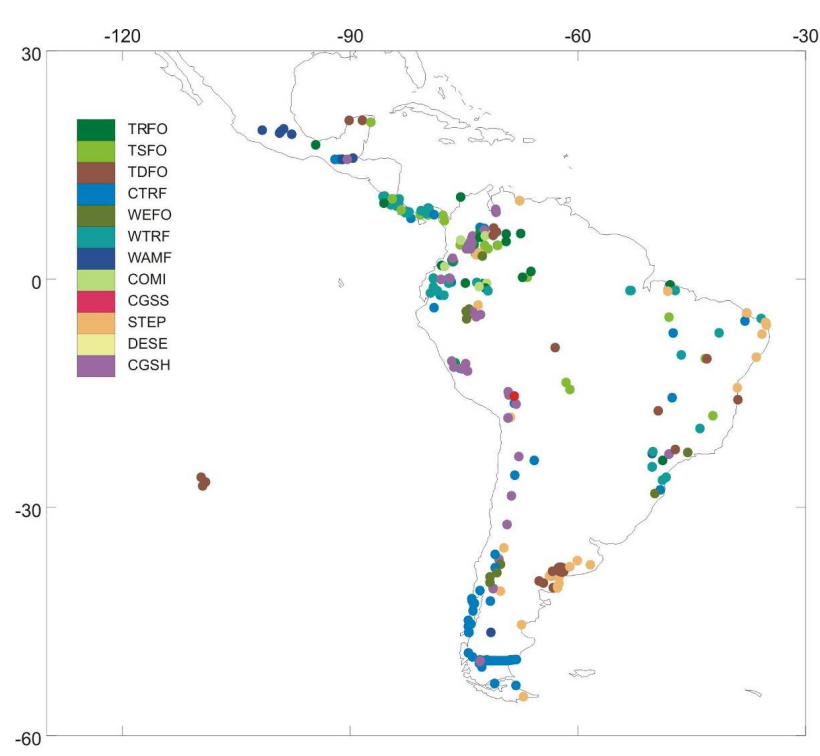

Fig. 7. Modern biomes reconstructed from surface pollen data (core top, trap, surface sediment) used to compare against the potential vegetation (Fig. 2).

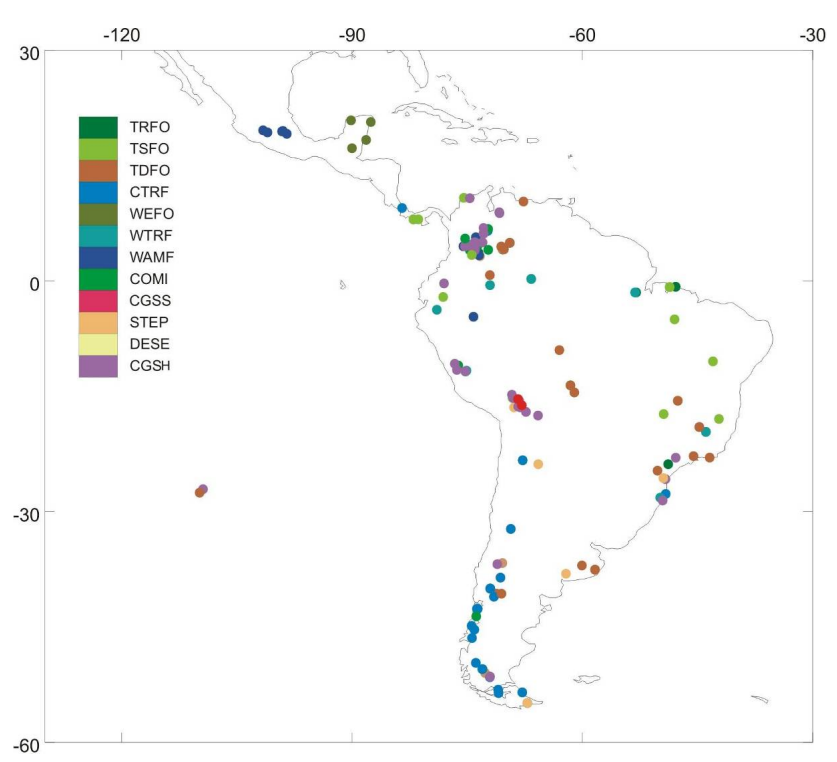

Fig. 8. Biome reconstruction at $6000 \pm 500{ }^{14} \mathrm{C}$ yr BP from radiocarbon dated fossil pollen.

or tropical seasonal forest reconstructed for the present. A site on the present southern Amazonian boundary (Laguna Chaplin) records tropical seasonal forest. Sites in southern South America nearly all show a transition from cool mixed forest biomes and cool mixed forest to cool grass/shrubland and cool grassland. However, within this homogenous reconstruction a number of sites have a relatively high affinity to the cool temperate rain forest biome, due to a mix of Donartia and Nothofagus pollen: this ex-

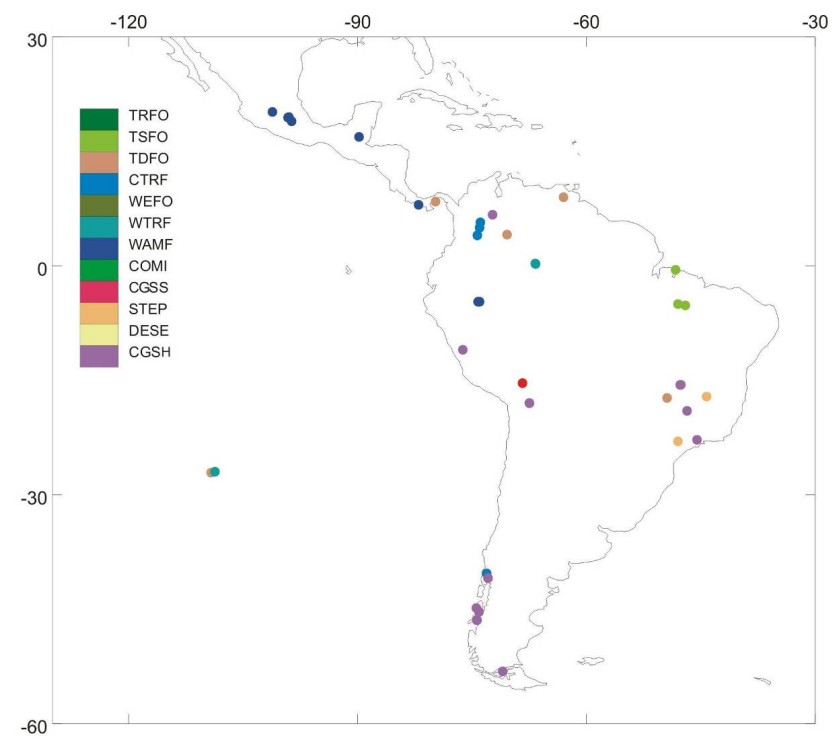

Fig. 9. Biome reconstruction at $18000 \pm 1000{ }^{14} \mathrm{C}$ yr BP from radiocarbon dated fossil pollen.

plains why the northernmost site in this cluster records cool temperate rain forest. Sites in southeastern Brazil record a transition from tropical dry forest to tropical seasonal forest and cool grass/shrubland. Sites in Amazonia record mainly tropical seasonal forest, warm temperate rain forest or steppe; this combination indicating relatively mesic forest. In the Colombian lowlands, tropical dry forest continues to be assigned whereas Colombian highland locations reflect a marked change from cool temperate rain forest and cool mixed forest to the cool grass/shrubland biome. Sites in Central America show a change from tropical seasonal forest to tropical dry forest, e.g. El Valle. The Mexican highland sites remain unchanged, continuing to support warm mixed forest with the pollen records being dominated by Pinus and Quercus.

\section{Discussion and conclusions}

\subsection{Modern reconstruction}

Previous applications of the biomisation method in Africa (Jolly et al., 1998a; Elenga et al., 2000), China (Yu et al., 1998, 2001), Australia (Pickett et al., 2004), Eastern North America (Williams et al., 2000), Eurasia (Tarasov et al., 1998a), Europe (Prentice et al., 1996a, b; Tarasov et al., 1998a, b; Elenga et al., 2000), Japan (Takahara et al., 2001) and Western North America (Thompson and Anderson, 2000) demonstrate that technique is able to translate multi-site pollen data to coarse resolution vegetation reconstructions that works well over a range of vegetation types. The Latin American results presented here provide a further test of this ability. The ability of the biomisation method to 
reconstruct biomes derives in part from the relatively coarse vegetation classification (Fig. 2); which conceals significant intra-biome variation; for example, we do not distinguish subtypes of the warm evergreen forest biome which contains Araucaria in southern and southeastern Brazil and Podocarpus in the northern Andes. The success of the biomisation technique is in part be due to reconstructions being carried out at a regional scale, allowing the methodology to be adapted to the local flora, bioclimatic gradients and pollen spectra. For example, the treatment of Quercus pollen in Latin America is quite different from that in a European context. Similarly, in Africa Podocarpus is assigned to the warm temperate broad-leaved evergreen PFT (Jolly et al., 2001), although this taxon is a coniferous needle-leafed tree, in Latin America it is assigned to cool and intermediate temperate conifers. This regional focus also allows the pollen to plant functional type allocations to be based on good ecological information concerned with environmental tolerances to growth limits and an understanding of how representative the pollen is of the surrounding vegetation. This is particularly important as the pollen taxa identified to the generic level (the taxonomic level usually identified to) exhibit considerable plasticity in their growth form and environmental tolerance. For example, within the genus Cordia, commonly a woody shrub of open thorn woodland, of the northern Andes (Cleef and Hooghiemstra, 1984) two species of Cordia are herbs in cerrado (Pereira et al., 1990; Sarmiento, 1975), the genus is also present (C. lomatoloba and $C$. sagotii) in Amazonian terra firme forest and Guyanese lowland rain forest (Steege, 1998). Furthermore the specific nature of pollen production, dispersal and incorporation into a sedimentary environment exhibits considerable variability that is part dependent on site characteristic. All these factors have a bearing on the results and need to be considered in the designing of the input matrices into the biomisation process and interpretation of results.

Biomes are mainly accurately reconstructed for the present-day even though large areas of Latin America are covered with vegetation that has been altered by a long history of human land use (Behling, 1996; Binford et al., 1987; Fjeldså, 1992; Gnécco and Mohammed, 1994; Gnécco and Mora, 1997; Marchant et al., 2004; Northrop and Horn, 1996). One possible reason for this may relate to the nature of the "modern" samples. Within our analysis the modern samples are largely derived from sedimentary columns rather than surface trap pollen data, and hence they may stem from the last 500 years and be reflective of a period prior to intensive human-induced change. However, the signal of vegetation clearance does impact on the modern reconstruction as shown by the large number of sites recording cool grass/shrubland, particularly at lower and mid-altitudes that should support cool mixed forest or cool temperate rain forest. These assignments are thought to result from human impact with the pollen spectra being dominated by Poaceae and hence recording more open vegetation. To quantify the nature of this impact, it is possible to tailor the biomisation methodology to include elements of the pollen spectra, such as agricultural and ruderal taxa, that may indicate human impact (Marchant et al., 2002). The ability to reconstruct potential, rather than actual vegetation, may also relate to the type of impact; although spatially relatively widespread, forest clearance is often only partial with many localised patches of forest and secondary vegetation remaining. This results in the floristic composition of the remaining vegetation, in palynological terms at least, closely reflecting the original vegetation composition. For example, the forest surrounding the Fúquene-II site is a successional type of forest whereas the natural vegetation would be a Andean forest type dominated by Quercus and Weimannia mixed with Croton, Oreopanax and Phyllanthus (Van Geel and Van der Hammen, 1973). In addition to the relatively coarse potential vegetation and biome classification, mapping the highest biome affinity score to each site as a single dot also allows the method to be relativity robust. Although this is suitable for the relatively coarse reconstructions necessitated by the continental/sub-continental scale, when investigating a small area, more information can be preserved from the analysis. Indeed at a regional scale information on sub-dominate biomes can be kept (Marchant et al., 2001a), new more defined biomes (Bigelow et al., 2003) or at a site-specific scale where the affinity scores to all the biomes can be retained (Marchant et al., 2001b, 2002b).

Despite the overall agreement between potential and reconstructed biomes a number of locations show anomalies. Due to the floristic and structural similarities between warm and cool grasslands (Tarasov et al., 1998a), grass-dominated biomes can be particularly difficult to distinguish from one another. Differentiation is possible by the other plants within steppe and cool grass/shrubland, although there remains a high affinity score to the cool grass/shrubland at low altitudes with the reverse for the steppe biome at high altitudes. Another facet is that some lowland sites show reconstructions of highland biomes, e.g. sites in central Panama and Amazonia recording warm temperate rain forest. This result is driven by the presence of genera that are typical of montane vegetation, e.g. Hedyosmum, Podocarpus and Quercus. A possible explanation for the presence of these highland elements is that they are relictual; relatively isolated today they were previously much more widespread under the glacial climate norm of the Quaternary. This suggestion is supported by the similarity, at a generic level, of the flora in highland Brazil and the northeastern Andes, and the isolated patches of savanna within Amazonian forest and the Brazilian cerrado. Furthermore, it is interesting that the presence of highland elements appears to be greater when the moisture levels are high. For example, within the Chocó Pacific region, where rainfall exceeds $15000 \mathrm{~mm} \mathrm{yr}^{-1}$, montane elements appear more common than within Amazonia (Cleef, personal communication). 
Notwithstanding some of the anomalies mentioned, the biomisation method applied to Latin American pollen data can reconstruct large-scale vegetation patterns despite many pollen taxa having different ecological interpretations under different environmental settings (Grabandt, 1980), representation of parent vegetation by pollen likely to be subject to inter-annual variability (Behling et al., 1997b), and tropical vegetation is difficult to reconstruct through pollen assemblages (Bush, 1991; Mancini, 1993; Bush and Rivera, 1998; Behling et al., 1997). These factors demonstrate the importance of basing the input matrices for the biomisation process on all the available ecological information that allowing for the multiple assignment of the pollen taxa to the PFTs.

\subsection{Late Quaternary biome change and palaeoenviron- mental interpretations}

\subsection{1 $6000 \pm 500{ }^{14} \mathrm{C} \mathrm{yr} \mathrm{BP}$}

Compared to the present, the sites at $6000 \pm 500{ }^{14} \mathrm{C} \mathrm{yr}$ $\mathrm{BP}$ record either the same biome or one indicating more xeric vegetation. Dry environmental conditions in southern Brazil extend from the early Holocene until approximately $4500{ }^{14} \mathrm{C}$ yr BP when there was an increase in arboreal taxa (Alexandre et al., 1999). Maximum aridity in southeast Brazil was reached between approximately 6000 and $5000{ }^{14} \mathrm{C}$ yr BP, prior to the transition to a modern climate (Behling, 1997a). The driest phase in central Brazil is at approximately $5000{ }^{14} \mathrm{C}$ yr BP; relatively moist climate conditions similar to today setting in after $4000{ }^{14} \mathrm{C}$ yr BP (Ledru, 1993; Marchant and Hooghiemstra, 2004). Although fire has been proposed as being responsible for late Holocene variation in the forest/savanna boundary in Brazil (Vernet et al., 1994; Desjardins et al., 1996; Horn, 1993), this relative aridity is thought to reflect an extended dry season during this period (Behling, 1997b). An extended dry season may explain why Araucaria-dominated forest were still restricted in their distribution relative to the modern day, not significantly increasing in range until approximately $3000{ }^{14} \mathrm{C}$ yr BP (Behling, 1997a). From our analysis the temporal perspective is missing, hence, we are unable to indicate if the vegetation reflects a stable dry period, or a period where there are alternating periods of dry and humid climates linked for example to El Niño activity (Martin et al., 1993; Sifeddine et al., 2001). A relatively dry phase is also recorded in northwestern Argentina between 7500 and $5800{ }^{14} \mathrm{C}$ yr BP (Schäbitz, 1991). Although pollen assemblages do not lend themselves well to distinguishing moisture from temperature changes, stable hydrogen isotope analysis on mosses show the vegetation of southern South America is highly sensitive to changes in moisture regime (Pendall et al., 2001). The predominance of steppe in southeastern Argentina agrees with the reconstruction by Prieto (1996): steppe characterising the area between 7000 and $5000{ }^{14} \mathrm{C}$ yr BP. Locally high moisture levels at sites closer to the Atlantic Ocean (Prieto,
1996) may explain why sites under strongest maritime influence (Aguads Emendadas, Cerro La China) changes from steppe to tropical dry forest as the local environment is able to support more arboreal taxa. In soutwestern Patagonia a sustained increase in Nothofagus pollen has been detected from around $6800 \mathrm{yr}$ BP thought to result from locally increased moisture levels (Villa-Martinez and Moreno, 2007) Locally increased moisture levels in this part of Latin America during the mid Holocene are though to stem from intensification of the southern Westerlies (Gilli et al., 2005).

Farther west, cool temperate rain forest assignments indicate a similar climatic regime and the maintenance of Valdivian rain forest (Villigran, 1988). A dry phase is also recorded at many Andean sites, for example, in northern Chile desiccation of the Puna ecosystem is recorded between 8000 and $6500{ }^{14} \mathrm{C}$ yr BP (Baied and Wheeler, 1993; Villigran, 1988). In lowland Chile, the period of maximum aridity occurred between 9400 and $7600{ }^{14} \mathrm{C}$ yr BP with drier than present conditions continuing until $5000{ }^{14} \mathrm{C}$ yr BP (Heusser, 1982), this could explain the increased presence of steppe at sites along the southern Andes. On the central Peruvian Andes, a dry warm climate was encountered between 7000 and $4000{ }^{14} \mathrm{C}$ yr BP (Hansen et al., 1994). $\delta^{18} \mathrm{O}$ measurements from an ice core record taken from highland Peru show that mid-Holocene climatic warming and drying was recorded from 8200 to $5200{ }^{14} \mathrm{C}$ yr BP with maximum aridity between 6500 to $5200{ }^{14} \mathrm{C}$ yr BP (Thompson et al., 1995). Farther north on the Bolivian Andes, a dry phase is recorded from approximately $5500{ }^{14} \mathrm{C}$ yr BP (Abbot et al., 1997). The slight increase in the number of arboreal biome assignments at northern Andean sites can be interpreted as an up-slope shift of forest line. This conforms to the suggestion based on pollen data by van Geel and van der Hammen (1973) that the vegetation zones in the northern Andes were several hundred of meters higher than the present at approximately $6000{ }^{14} \mathrm{C}$ yr BP. Relatively dry conditions have also been indicated for lowland Colombia for the mid-Holocene although the onset of dry conditions varied considerably between sites - occurring between 6500 and $4500{ }^{14} \mathrm{C}$ yr BP (Behling et al., 1999). Added complexity is caused by steep environmental gradients associated with non-climatic factors. For example, the presence of the tropical dry forest biome in lowland Colombia, e.g. the catchment of El Piñal, results from a combination of strongly seasonal conditions at present and locally strong edaphic influence (Behling and Hooghiemstra, 1999).

Farther north, the assignment of Lake Valencia to the tropical dry forest is in agreement with the site-specific interpretation that more arboreal taxa (Bursera, Piper and Trema) were present after approximately $10000{ }^{14} \mathrm{C}$ yr BP due to the onset of a more humid climate (Bradbury et al., 1981): these tropical raingreen taxa are indicative of a seasonal climate with relatively dry conditions. This appears to be a regional signal as early Holocene evergreen forests of northern Venezuela were replaced by semi-deciduous elements during the mid-Holocene (Leyden, 1984). Enhanced precipitation 
over Central America would have been accompanied by a northward shift of the ITCZ, enhanced southerlies and cooler equatorial sea surface temperatures (Harrison et al., 2003). Low lake levels in central Panama also indicate that environmental conditions at this period were more xeric (Piperno et al., 1991b; Bush et al., 1992) whereas sites on the Yucatán peninsula show a shift to warm evergreen forest where the warmer conditions that characterise the early Holocene persisted until approximately $6500{ }^{14} \mathrm{C}$ yr BP (Brown, 1985). This result may stem from locally high moisture levels as a result of maritime influence, a similar mechanism having being proposed to explain a comparable shift in coastal Brazil and Argentina. Despite the majority of the evidence for a mid-Holocene dry period, there still remains a debate about the intensity, and even the occurrence, of this. SalgadoLabouriau et al. (1998) suggests that most savanna areas were characterised by increased rainfall between 7000 and $6000{ }^{14} \mathrm{C}$ yr BP although there is considerable variation in the timing of the onset of more humid conditions so it may be that such a mesic period falls outside our temporal window.

One of the main mechanisms used to explain moisture shifts is fluctuations in the Southern Oscillation and the migration of the ITCZ (Martin et al., 1997). Martin et al. (1997) suggests that during the mid-Holocene, the ITCZ was located farther north than its present-day position (Fig. 1) this would produce a summer rainfall deficit and increased winter precipitation; in short greater seasonality. Rather than changes in the median position of the ITCZ, changes in the character of the ITCZ oscillation, such as greater latitudinal range for annual migration, can be invoked to explain vegetation changes (Behling and Hooghiemstra, 2001). However, due to the topographical influence of the Andes and the convergence of westerly and easterly winds, the ITCZ has a sinusoidal profile over northern South America (Fig. 1). Therefore, moisture changes over northeastern South America are likely to result from the importance of convective moisture sources; reduced precipitation, particularly in mid latitude western South America, following reduced intensity of westerly climate systems. It is also possible that episodic dry events that presently occur in South America in relation to sea-surface temperature anomalies of the Pacific Ocean (ENSO) were more frequent in the mid-Holocene (Markgraf, 1998). This later suggestion may also have led to the increased fire frequency indicated in southeast Brazil (Alexandre et al., 1999).

The spatial relationship between Latin American and Africa (Servant et al., 1993; Jolly et al., 1998a) warrants further investigation (Marchant and Hooghiemstra, 2004). A particular target for the investigation could be the impact and feedbacks of vegetation changes on climate. For example, large changes in African vegetation about the Sahel are suggested to have been important in influencing Indian monsoon dynamic (Doherty et al., 2000). Such a phenomena of vegetation feedbacks on the climate system appears weaker in
South America than in Africa although it is likely to have had an impact as yet unqualified. Certainly Latin America would benefit from targeted model applications in the same way that has been applied to Africa (Kubatzki and Claussen, 1998; Doherty et al., 2000). This modelling of climate dynamics Latin American represents a special challenge for climate models and modellers (Valdes, 2000) primarily due to the steep environmental gradients and rapid transition from one biome to another (Fig. 2).

\subsection{2 $18000 \pm 1000{ }^{14} \mathrm{C} \mathrm{yr} \mathrm{BP}$}

The dating of the LGM in Latin America can be problematic (Bush et al., 1990; Hooghiemstra et al., 1992; Ledru et al., 1996, 1998; Sifeddine et al., 2001); late Pleistocene sediments often containing sedimentary gaps at, or about, the LGM (Ledru et al., 1998), that are compounded by slow sedimentation rates. These sedimentary constraints make characterisation of the LGM vegetation highly contentious and have fuelled debates on LGM climates spanning two decades (Hooghiemstra and van der Hammen, 1998; Colinvaux et al., 2000; Thomas, 2000). Indeed, it has been suggested that some of the sites used in our analysis do not contain a sedimentary record of the LGM (Ledru et al., 1998) although due to application of a 2000 year-wide time window, we are able to include some of these sites with contentious sediments.

The LGM in Latin America, like most of the tropics, was characterised by a cold dry climate (González et al., 2008) with temperature reduced by about $4^{\circ} \mathrm{C}$ and precipitation by about $30 \%$ (Farrera et al., 1999). Ice caps were present on the southern tip of South America which spread onto the plains and the coastal area (Heine, 1995). Evidence from glacial moraines also indicates considerable expansion of Andean glaciers (Hollis and Schilling, 1981; Villagran, 1988; Birkland et al., 1989; Seltzer, 1990; Thouret et al., 1997). Most of southern South America was characterised by an erosional environment; locations that would later accumulate sediments were glaciated, or subject to fluvial activity (Heine, 2000). This situation is recorded by ice cores from the high Andes that contain large amounts of dust about the LGM, this being derived from surrounding deflating desert areas (Thompson et al., 1995). This cold, arid environment is clearly reproduced by the vegetation which shows a transformation from the cool temperate rain forest to the cool grass/shrubland biome. Although Nothofagus-dominated forest is thought to have been extirpolated from coastal Chile at the LGM (Hollis and Schilling, 1981), fossil beetle assemblages in basal peat from Puerto Eden $\left(49^{\circ} \mathrm{S}, 74^{\circ} \mathrm{W}\right)$ indicate that Nothofagus-dominated forest survived glaciation within the Chilean channels (Ashworth et al., 1991). An earlier date of deglaciation of the Taitao Peninsula indicates there was relatively local migration from Chiloé Island that may explain the rapid re-growth of Nothofagus-dominated forest (Lumley and Switsur, 1993). Along the Chilean Pacific coast the present cool evergreen 
forest was shifted approximately $5^{\circ}$ northwards relative to the present day; not as a discrete forest type but as a parkland type vegetation mosaic (Villagran, 1988), not forming closed forests until the early Holocene (Schäbitz, 1994; Heusser, 1995). This vegetation is evidenced with the analysis presented here by the northernmost site (Laguna Six Minutes) recording cool temperate rain forest. However, it is unlikely this represents closed forest persisting in the area, trees being present within a woodland/steppe vegetation mosaic (Villagran, 1988). The rate of spreading of this forest into the Holocene would probably have been strongly dependent on the density of the parent plants from the initial seeding fraction (Huntingford et al., 2000). The maintenance of cool temperate rain forest taxa, albeit at relatively low levels, may result from high moisture levels as recorded by high lake stands at this time (Markgraf et al., 2000). These may reflect outbreaks of polar air and subsequent generation of low-pressure systems in the western Atlantic; combined with lower temperatures this situation would lead to a positive water balance. Indeed, the presence of relatively local moisture sources would have been important at the LGM and allow us to explain regional patterns of biome change outside the influence of the ITCZ migrations (Markgraf et al., 2000).

Considering the sites along the northern Andes, it is clear from the vegetation that climate was colder during the LGM, reductions up to $12^{\circ} \mathrm{C}$ may have been reached at very high altitudes (Thompson et al., 1995). A substantial temperature depression during the last glacial period is mirrored by a significant impact on the vegetation composition and distribution. From our analysis it is apparent that the tree line was significantly lower at the LGM, concordant with a suggested lowering of vegetation zones by approximately 1000 to $1500 \mathrm{~m}$ relative to the present-day position (Monslave, 1985; Wille et al., 2001). At lower elevations in western Colombia, a more conservative depression of the vegetation has been suggested from Timbio (Wille et al., 2000). Indeed, the spatial character of the cooling and drying in the Neotropics is still under debate (Markgraf, 1993; Colinvaux, 1996; Hooghiemstra and Van der Hammen, 1998; Farrera et al., 1999; Boom et al., 2002). Greater temperature change at high altitudes compared with those at low altitudes and at the sea surface (CLIMAP, 1976) can be explained in terms of changes in lapse rate (Bush et al., 1990; Peyron et al., 2000; Wille et al., 2001) or compression of vegetation belts (Van der Hammen and Absy, 1994). The lapse-rate gradient is partly influenced by atmospheric moisture levels (Barry and Chorley, 1990). As precipitation was reduced at the LGM, an overall steeper lapse rate, particularly at higher altitudes where moisture reductions would have been highest, seems likely (Wille et al., 2001). The extent to which lapserate changes can be used to explain spatially different signals from the data must be used with caution, particularly as most palaeoclimatic reconstructions have been carried out with some kind of modern analogue approach (Farrera et al., 1999). These reconstructions commonly do not take into ac- count non-climatic parameters which would impact on vegetation composition and distribution such as volcanic activity (Kuhry, 1988), fire (Cavelier et al., 1998; Rull, 1999), UVB insolation (Flenley, 1998) or atmospheric composition, in particular changing $\mathrm{CO}_{2}$ levels (Woodward and Bond, 2004). For example, concentrations of $\mathrm{CO}_{2}$ reduced to glacial levels $(200 \mathrm{ppmV})$ have been shown to have a very significant impact on tropical vegetation (Jolly and Haxeltine, 1997; Boom et al., 2002; Marchant et al., 2002b; Wu et al., 2007a, b).

In south-east Brazil vegetation at the LGM was characterised by tropical dry forest and tropical seasonal forest; this latter vegetation type may have been restricted within deep valleys and along waterways; site-specific records from southeast Brazil indicate open grasslands (campo limpo) with forest elements being retained as gallery forest (Behling, 1997a). Some model reconstructions of global vegetation patterns have indicated that there was an increase in warm evergreen forest in Brazil at the LGM at the expense of tropical seasonal forest (Prentice et al., 1993). This pattern of change is supported by the data presented here where plants generally found at high altitudes today were more common in Amazonia at the LGM. Clapperton (1993) used geomorphic data to infer a very sparsely vegetated landscape on the Brazilian Highlands, possibly relating to our reconstruction of steppe for a site in eastern Brazil. However, the cool grass/shrubland biome appears to be a common type of vegetation at this time. Cold climates in eastern South America could have resulted from the incursion of polar cold fronts that would occasionally reach northwards of the equator (Ledru, 1993; Behling and Hooghiemstra, 2001). This phenomenon could combine with equatorward shift of polar high-pressure areas and mid-latitude cyclones resulting in displacement and compression of the subtropical anticyclone between mid latitudes westerlies (Dawson, 1992). This climatic regime would result in more restricted migration of the ITCZ and pronounced aridity that would have been compounded by lower sea surface temperatures and associated reduction in atmospheric moisture.

At altitudes of approximately $3000 \mathrm{~m}$ in northern Peru vegetation about the LGM comprised a mixture of wet and moist montane forest elements with open woodland (Hansen and Rodbell, 1995); this vegetation association having no modern analogue. Although the Andes remained relatively moist at the LGM, particularly in the northern part where the concave shape of the mountain chain entrap moisture from the rising air (Fjeldså et al., 1997), it is not certain what occurred in the lowland areas to the east of the Andes (Colinvaux, 1989; Colinvaux et al., 1997; Bush et al., 1990; Thouret et al., 1997). In the Colombian lowlands two sites are characterised by the tropical dry forest biome, this agrees with the suggestion from a pollen study at Rondonia that very open savanna characterised the catchment at the LGM (van der Hammen and Absy, 1994). Similarly, sparse vegetation cover would have been present on the Plateau of Mato Grosso (Servant et al., 1993) and is likely to have extended along the 
coastal areas of Guyana and Surinam (Wijmstra, 1971) - this scenario is supported by our analysis, i.e. a site in lowland Panama recording tropical seasonal forest. Although the majority of the area presently covered by drier types of tropical forest would probably have been replaced by more open woodland at the LGM, environmental changes in savannas at the LGM appear to have been spatially complex. Whether the drier, cooler, conditions resulted in restricted range forest refugia cannot be answered from the available evidence although the vegetation appears heterogeneous as a mosaic of Andean, savanna and tropical rain forest taxa combined. Indeed, this reiterates the suggestion by Colinvaux et al. (2001) and now widely accepted within the palaeoecological community, that plants responded to Quaternary climate changes as individuals not as biomes. Therefore, to fully investigate vegetation response to climate change is necessary to retain information contained within the affinity scores to the subdominant biomes (Marchant et al., 2002), or to carry out the analysis at the PFT level. Indeed this approach would allow investigations into which elements of the vegetation were particularly sensitive to environmental change. Expansion of savanna could have been aided by reduced $\mathrm{CO}_{2}$ concentrations and the resultant competitive advantage attained by $\mathrm{C}_{4}$ grasses over $\mathrm{C}_{3}$ plants (Haberle and Maslin, 1999; Marchant et al., 2002).

Within highland México, warm mixed forest continues to be reconstructed due to the presence of Pinus and Quercusdominated forests. Although the same biome is recorded at all time periods, it unlikely to be analogous to present day mixed forest; this was characterised by sparsely forested temperate scrub (Binford et al., 1987). This continued reconstruction also could be demonstrating that certain locations are unlikely to register a change due to their position in bioclimatic space: those sites located far away from the boundaries of adjacent biomes would need a massive climatic change to result in an ecosystem shift. Such a situation has clearly been shown for the Holocene where the sites to undergo biomisation show little change relative to the modern (Ortega-Rosas et al., 2008a, b). Indeed, a site located in northwestern México records a transition from cool conifer forest to warm mixed forest about $6000 \mathrm{yr}$ BP (Ortega-Rosas et al., 2008b). Similarly, a strong aridity signal is directly recorded by low lake levels in central México due to reduced northern excursion of the ITCZ, trade wind circulation, and ensuing reduced oceanic-land moisture transfer (Markgraf et al., 2000) that would have been reflected in ecosystem response. For example, forest on the Pacific side of the Central America contained a mosaic of high and low altitude forest species; a similarly novel type of forest has also been shown for Mera, Ecuador (Liu and Colinvaux, 1985) and Peten, Guatemala (Leyden, 1984). Of the two sites that record the warm evergreen forest biome at this period a site in Guatemala was dominated by Chenopodiaceae, Juniperus, Pinus and Quercus.
We have presented vegetation reconstructions throughout Latin America at $6000 \pm 500{ }^{14} \mathrm{C}$ yr BP and $18000 \pm 1000$ ${ }^{14} \mathrm{C}$ yr BP using an objective method based on biomes, constituent PFTs that are described by a set of unique pollen spectra. As a unified methodology has been applied to the pollen data, this reconstruction of biomes provides an objective basis for interpreting large-scale vegetation dynamics, and the environmental controls on these over the late Quaternary and can be used as a dataset for model-data comparisons at 6000 and $18000 \mathrm{yr}$ BP. Changes at $6000 \pm 500$ ${ }^{14} \mathrm{C}$ yr BP, although relatively small, indicate a transition to more xeric vegetation. The changes at $18000 \pm 1000{ }^{14} \mathrm{C}$ yr $\mathrm{BP}$ are more homogenous and indicative of a cooler, drier climate. These reconstructions are consistent with numerous site-specific interpretations of the pollen data. The success of the reconstruction has in part been determined by the coarse resolution of biome definitions, and using the most dominant biome for description and interpretation of the results. To develop understanding of vegetation response to environmental change, and possible feedbacks, information that is presently redundant should be retained and the results combined with climate/vegetation modelling initiatives. It is apparent from the relatively sparse coverage, in comparison to Europe and North America, that the late Quaternary vegetation history of the Neotropical phytogeographical realm remains still relatively poorly resolved despite its importance in model testing, developing biogeographical theory (Tuomisto and Ruokolainen, 1997), and understanding issues concerned with biodiversity and human-environment interactions. It has been shown that environmental change is rarely spatially uniform and as such necessitates an even greater number of sites to determine more precisely this complexity and the driving mechanisms behind this. New sites, located in key areas, combined with the application of a range of proxies of environmental change, are required to refine our understanding of Neotropical ecosystem responses to late Quaternary climatic variations.

Acknowledgements. This research has been funded by the Netherlands Organisation for Scientific Research (NWO) under award 750:198:08 to Henry Hooghiemstra/Rob Marchant. Rob Marchant recent contribution being supported by EU Grant No: EU-MXC-KITE-517098. This paper contributes to the BIOME 6000-research initiative which is a community-wide collaboration that started in 1994 under four elements of the International Geosphere-Biosphere Program (IGBP-GAIM, IGBP-DIS, IGBPGCTE and IGBP-PAGES) with the aim to create fully documented pollen and plant macrofossil data sets for 6000 and $18000{ }^{14} \mathrm{C} \mathrm{yr}$ BP. Dominique Jolly was instrumental in developing and applying these techniques in Africa and subsequent application to different geographical regions - indeed the Latin American application presented here would not have been possible without this massive contribution and encouragement throughout the development of this work. In particular we thank Sandra Diaz, Liz Pickett, Colin Prentice and Bob Thompson for comments on earlier drafts of this manuscript. C. Peñalba and an anonymous reviewer are thanked for insightful reviews that have improved the manuscript 
and some of the associated figures. Ary Teixeira de Oliveira-Filho and Olando Rangel are particularly thanked for comments on the ecology of the Latin American taxa. Kirsten Sickel, Gerhard Boenish and Silvana Schott are thanked for help in producing the figures and archiving the results. Particular thanks must go to Eric Grimm, John Keltner and Vera Markgraf for their energies in establishing, and developing, the Latin American Pollen Database. At the very centre of this work are all those palynologists who have supplied data to the LAPD, or specifically to be used for this paper; without their efforts such syntheses would not be possible.

Edited by: R. Bonnefille

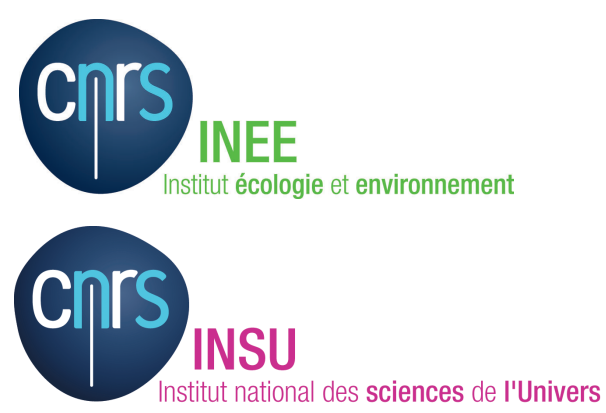

Publication of this paper was granted by EDD (Environnement, Développement Durable)

and INSU (Institut des Sciences de l'Univers) at CNRS.

\section{References}

Abbot, M. B., Sletzer, G., Kelts, K. R., and Southon, J.: Holocene paleohydrology of the tropical Andes from lake records, Quatern. Res., 47, 70-80, 1997.

Absy, M. L.: A palynolgical study of Holocene sediments in the Amazon basin. Dissertation, University of Amsterdam, The Netherlands, 1979.

Absy, M. L., Cleef, A., Fournier, M., Servant, M., Siffedine, A., Da Silva, M. F., Soubies, F. Suguio, K., Turcq, B., and van der Hammen, T.: Mise en évidence de quatre phases d'ouverture de la forêt dense dans la sud-east de l'Amazonie au cours des 60000 dernières années. Première comparison avec d'autres regions tropicales, Competes Rendues Academie Science Paris, 313, 673-678, 1991.

Alexandre, A., Meunier, J. D., Mariotti, A., and Soubies, F.: Late Holocene phytolith and carbon-isotope record from a latosol at Salitre, South-Central Brazil. Quatern. Res., 51, 187-194, 1999.

Almeida, L.: Vegetación, fitogeographica y paleoecologíca del zacatonal alpine y bosques montanos de la región central de México, PhD thesis, University of Amsterdam, 1997.

Armesto, J. J., Villágran, C. Aravena, J. C., Pérez, C., SmithRamirez, C., Cortés, M., and Hedin, L.: Conifer forests of the Chilean coastal ranges, Ecology of Southern Conifers, edited by: Enright, N. J. and Hill, R. S., Melbourne University Press, 1995.

Ashworth, A. C. and Markgraf, V.: Climate of the Chilean Channels between 11,000 and 10,000 yr B.P. based on fossil pollen and beetle analysis, Revista Chilena de Historia Natural., 62, 61-74, 1989.

Ashworth, A. C., Markgraf, V., and Villagran, C.: Late Quaternary climatic history of the Chilean channels based on fossil pollen and beetle analyses, with an analysis of the modern vegetation and pollen rain, Jn Quatern. Sci., 6, 279-291, 1991.

Argollo, J. and Mourguiart, P.: Late Quaternary climate history of the Bolivian Altiplano, Quatern. Int., 72, 37-51, 2000.

Baied, C. A. and Wheeler, J. C.: Evolution of high Andean Puna ecosystems: environment, climate, and cultural change over the last 12,000 years in the central Andes, Mount. Res. Dev., 13, 145-156, 1993.

Barry, P. and Chorley, L.: Mountain weather and climate, Methuen, London and New York, 1990.

Beard, J. S.: The classification of tropical American vegetation, Jn. Ecol., 36, 89-100, 1955.

Behling, H.: Untersuchungen zur spätpleistozänen und Holozänen Vegetations und Klimageschichte der tropische Kustenwälder und der Araukarienwälder in Santa Catarina (Süd Brazilien), Dissertations Botanicae Band 206, J. Cramer, Berlin Stuttgart, 1993.

Behling, H.: First report on new evidence for the occurrence of Podocarpus and possible human presence at the mouth of the Amazon during the Late-glacial, Veg. His. Archaeobot., 5, 241246, 1996.

Behling, H.: Late Quaternary vegetation, climate and fire history from the tropical mountain region of Morro de Itapeva, SE Brazil, Palaeogeog., Palaeoclim., Palaeoecol. 129, 407-422, 1997a.

Behling, H.: Late Quaternary vegetation, climate and fire history of the Araucaria forest and campos region from Serra Campos Gerais, Paraná State (South Brazil), Rev. Palaeobot. Palynol., 97, 109-121, 1997b.

Behling, H., Berrio, J. C., and Hooghiemstra, H.: Late Quaternary pollen records from the middle Caquetá River basin in central Colombian Amazon. Palaeogeog., Palaeoclim., Palaeoecol., 145, 193-213, 1999.

Behling, H. and Hooghiemstra, H.: Late Quaternary palaeoecology and palaeoclimatology from pollen records of the savannas of the Llanos Orientales in Colombia, Palaeogeog., Palaeoclim., Palaeoecol., 139, 251-267, 1998.

Behling, H. and Hooghiemstra, H.: Environmental history of the Colombian savannas of the Llanos Orientales since the Last Glacial Maximum from lake records El Piñal and Carimagua, Jn. Paleolim., 21, 461-476, 1999.

Behling, H. and Hooghiemstra, H.: Holocene Amazon rain forestsavanna dynamics and climatic implications: high-resolution pollen record from Laguna Loma Linda in eastern Colombia, Jn Quatern. Sci., 15, 687-695, 2000.

Behling, H. and Hooghiemstra, H.: Neotropical savanna environments in space and time: Late Quaternary inter-hemispheric comparisons, in: Inter-hemispheric climate linkages, edited by: Markgraf, V., Academic Press, pp. 307-323, 2001.

Behling, H., Hooghiemstra, H., and Negret, A. J.: Holocene history of the Chocó rain forest from Laguna Piusbi, Southern Pacific Lowlands of Colombia, Quatern. Res., 50, 300-308, 1999.

Behling, H., Keim, G., Irion, G., Junk, W., and Nunes de Mello, J.: Holocene changes in the Central Amazon Basin inferred from Lago Calado (Brazil), Palaeogeog., Palaeoclim., Palaeoecol., 173, 87-101, 2001.

Behling, H. and Lima da Costa, M.: Holocene vegetational and environmental changes from the Lago Crispim record in northeastern Para State, eastern Amazonia, Rev. Palaeobot. Palynol., 114, 
145-155, 2001.

Behling, H., Negrelle, R. B., and Colinvaux, P.: Modern pollen rain from the tropical Atlantic rain forest, Reserve Volta Velha, South Brazil, Rev. Palaeobot. Palynol., 97, 287-299, 1997.

Behling, H., Negret, A. J., and Hooghiemstra, H.: Late Quatrnary vegeteational and climatic change in the Popayan region, southern Colombian Andes, Jn Quatern. Sci., 13, 43-53, 1999.

Bennet, K. D., Haberle, S., and Lumley, S. H.: The Late GlacialHolocene transition in southern Chile, Science, 290, 325-327, 2000.

Berrio, J. C., Hooghiemstra, H., Behling, H., and Van der Borg, K.: Late Holocene history of savanna gallery forest from Carimagua area, Colombia, Rev. Palaeobot. Palynol., 111, 295-308, 2000.

Berrio, J. C., Behling, H., and Hooghiemstra, H.: Tropical rain forest history from the Colombian Pacific area: a 4200-yr pollen record from Laguna Jotaordó, The Holo., 10, 749-756, 2000.

Berrio, J. C., Hooghiemstra, H., Behling, H., Botero, P., and Van der Borg, K.: Late Quaternary savanna history of the Colombian Llanos Orientales from Lagunas Chenevo and Mozambique: a transect synthesis, The Holo., 12, 35-48, 2002.

Berrio, J. C., Boom, A., Botero, P., Herrera, L., Hooghiemstra, H., Romero, F., and Sarmiento, G.: Multi-interdisciplinary evidence of The Holocene history of a cultivated floodplain area in the wetlands, Veg. His. Archaeobot., 10, 161-174, 2002.

Berrio, J. C., Hooghiemstra, H., Marchant, R., and Rangle, O.: Late glacial and Holocene history of dry forest in the Cauca Valley from sites Quilichao and La Teta, Jn Quatern. Sci., 17, 667-682, 2002.

Bigelow, N. H., Brubaker, L. B., Edwards, M. E., Harrison, S. P., Prentice, I. C., Anderson, P. M., Andreev, A. A., Bartlein, P. J., Christensen, T. R., Cramer, W., Kaplan, J. O., Lozhkin, A. V., Matveyeva, N. V., Murray, D. V., McGuire, A. D., Razzhivin, V. Y., Ritchie, J. C., Smith, B., Walker, D. A., Gajewski, K., Wolf, V., Holmqvist, B. H., Igarashi, Y., Kremenetskii, K., Paus, A., Pisaric, M. F. J., and Vokova, V. S.: Climate change and Arctic ecosystems I. Vegetation changes north of $55^{\circ} \mathrm{N}$ between the last glacial maximum, mid-Holocene and present, J. Geophys. Res., 108, 1-17, 2003.

Binford, M. W., Brenner, M., Whitmore, T. J., Higuera-Gundy, A., Deevey, E. S., and Leyden, B. W.: Ecosystems, palaeoecology and human disturbance in subtropical and tropical America, Quatern. Sci. Rev., 6, 115-128, 1987.

Birkland, P. W., Rodbell, D. T., and Short, S. K.: Radiocarbon dates on deglaciation, Cordillera Central, Northern Peruvian Andes, Quatern. Res., 32, 111-113, 1989.

Björck, S., Håkansson, H., Olsson, S., Barnekow, L., and Janssens, J.: Palaeoclimatic studies in South Shetland Islands, Antarctica, based on numerous stratigraphic variables in lake sediments, J. Palaeolim., 8., 233-272, 1993.

Boom, A., Marchant, R. A., Hooghiemstra, H., and Sinninghe Damste, J. S.: $\mathrm{CO}_{2}$ and temperature-controlled altitudinal shifts of $\mathrm{C}_{4}$ - and $\mathrm{C}_{3}$ - dominated grasslands allow reconstruction of $\rho \mathrm{CO}_{2}$, Palaeogeog., Palaeoclim., Palaeoecol., 177, 29-45, 2002.

Braconnot, P., Otto-Bliesner, B., Harrison, S., Joussaume, S., Peterchmitt, J.-Y., Abe-Ouchi, A., Crucifix, M., Driesschaert, E., Fichefet, Th., Hewitt, C. D., Kageyama, M., Kitoh, A., Lan, A., Loutre, M.-F., Marti, O., Merkel, U., Ramstein, G., Valdes, P., Weber, S. L., Yu, Y., and Zhao, Y.: Results of PMIP2 coupled simulations of the Mid-Holocene and Last Glacial Maximum -
Part 1: experiments and large-scale features, Clim. Past, 3, 261277, 2007, http://www.clim-past.net/3/261/2007/.

Bradbury, J. P., Leyden, B., Salgado-Labouriau, M. L., Lewis Jr., W. M., Schubert, C., Binford, M. W., Schubert, C. Binford, M. W., Frey, D. G., Whitehead, D. R., and Weibezahn, F. H.: Late Quaternary environmental History of Lake Valencia, Venezuela, Science, 214, 1299-1305, 1981.

Brown, B.: A summary of Late Quaternary pollen records from México west of the Isthmus of Tehuantepec, Pollen records of Late Quaternary North American Sediments, edited by: Bryant, V. M. and Holloway, R. G., American Association of Stratigraphic Palynologists Foundation, Dallas, Texas, 1985.

Brown, S. and Lugo, A. E.: Tropical secondary forests, Jn. Trop. Ecol., 6, 1-32, 199.

Bush, M. B.: Modern pollen-rain data from South and Central America: a test of the feasibility of fine-resolution lowland tropical palynology, The Holo., 1, 162-167, 1991.

Bush, M. B.: Neotropical plant reproductive strategies and fossil pollen representation, The American Naturalist, 145, 594-609, 1995.

Bush, M. B. and Colinvaux, P.: A 7000-year pollen record from the Amazon lowlands, Ecuador. Vegetatio., 76, 141-154, 1988.

Bush, M., Colinvaux, P., Wiemann, M. C., Piperno, D. R., and Liu, K.: Late Pleistocene temperature depression and vegetation change in Ecuadorian Amazonia, Quatern. Res., 34, 330-345, 1990.

Bush, M. B., Miller, M., de Oliveira, P. E., and Colinvaux, P.: Two histories of environmental change and human disturbance in eastern lowland Amazonia, The Holo., 10, 543-553, 2000.

Bush, M., Piperno, D. O., Colinvaux, P., Oliveria, P. E., Krissek, L. A., Miller, M. C., and Rowe, W. E.: A 14,300-yr palaeoecological profile of a lowland tropical lake in Panama, Ecol. Mono., 62, 251-275, 1992.

Bush, M. and Rivera, M.: Pollen dispersal and representation in a Neotropical rain forest, Global Ecol. Biogeog. Lett., 7, 379-392, 1998.

Björck, S., Håkansson, S. H., Olsson, S., Barnekow, L., and Janssens, J. A.: Palaeoclimatic studies in South Shetland Islands, Antarctica, based on numerous stratigraphic variables in lake sediments, J. Paleolim., 8, 233-272, 1993.

Byrne, R. and Horn, S. P.: Prehistoric agriculture and forest clearance in the Sierra de los Tuxtlas, Veracruz, México, Palynology, 13, 181-194, 1989.

Cavelier, J., Aide, T. M., Santos, C., Eusse, A. M., and Dupuy, J. M.: The savannization of moist forests in the Sierra Nevada de Santa Marta, Colombia, J. Biog., 25, 901-912, 1998.

Cerverny, R. S.: Present climates of South America, in: Climates of the Southern Continents: Present, Past and Future, edited by: Hobbs, J. E., Lindesay, J. A., and Bridgeman, H. A., J. Wiley and Sons, Chichester, 1998.

Clapperton, C. M.: Quaternary geology and geomorphology of South America. Elsevier, Amsterdam, 1993.

Claussen, M.: On coupling global climate models with climate models, Clim. Res., 4, 203-221, 1994.

Claussen, M. and Esch, M.: Biomes computed from simulated climatologies, Clim. Dynam., 9, 235-243, 1994.

Cleef, A. M. and Hooghiemstra, H.: Present vegetation of the area of the high plain of Bogotá, in: Vegetation and Climatic History 
of the High Plain of Bogotá, Colombia, edited by: Hooghiemstra, H., Ganter Verlag, Vaduz. Dissertationes Botanicae, 79, 1-368, 1984.

CLIMAP Project Members: The surface of ice-age Earth, Science, 191, 1131-1137, 1976.

Colinvaux, P. A.: Ice-age Amazon revisited, Nature, 340, 188-189, 1989.

Colinvaux, P. A.: Quaternary Environmental History and Forest Diversity in the Neotropics, in: Evolution and Environment in Tropical America, edited by: Jackson, B. C., Budd, A. F., and Coates, A. G., The University of Chicago Press, Chicago, London, 1996

Colinvaux, P. A., Bush, M., Steinitz-Kannan, M., and Miller, M. C.: Glacial and postglacial pollen records from the Ecuadorian Andes and the Amazon, Quatern. Res., 48, 69-78, 1997.

Colinvaux, P. A., De Oliveira, P. E., Moreno, J. E., Miller, M. C., and Bush, M.: A long pollen record from Lowland Amazonia: Forest and cooling in glacial times, Science, 274, 85-88, 1996.

Colinvaux, P. A., de Oliveira, P. E., and Bush, M. B.: Amazonian and neotropical communities on glacial time-scales: the failure of the aridity and refuge hypothesis, Quatern. Sci. Rev., 19, 141$169,2000$.

Colinvaux, P. A., Frost, M., Frost, I., Liu, K.-B., and SteinitzKannan, M.: Three pollen diagrams of forest disturbance in the western Amazon basin, Rev. Palaeobot. Palynol., 55, 83-99, 1988.

Cuatrecasas, J. and Barreto, A. J.: Pàramos. Villegas editores, Bogotá, Colombia, 1988.

De Oliveira, P. E.: A palynological record of Late Quaternary vegetation and climatic change in southeastern Brazil, PhD Thesis, Ohio State University, Colombus, OH, 1992.

De Oliveira, P. E., Barreto, A. M. F., and Suguio, K.: Late Pleistocene/Holocene climatic and vegetational history of the Brazilian caatinga: the fossil dunes of the middle Sao Francisco River, Palaeogeog., Palaeoclim., Palaeoecol., 152, 319-337, 1999.

Dawson, A. G.: Ice Age Earth: Late Quaternary Geology and Climate, Routledge, 1992.

Desjardins, T., Carneiro-Filho, A., Mariotti, A., Chauvel, A., and Girardin, C.: Changes of the forest-savanna boundary in Brazilian Amazonia during The Holo. revealed by stable isotope ratios of soil organic carbon, Oecologia, 108, 749-187, 1996.

Dominquez, G., Rojo-Vazquez, J. R., Galvan-Pina, V., and AguilarPalomino, B.: Changes in the structure of a coastal fish assemblage exploited by a small scale gillnet fishery during an El NiñoLa Niña event, Est. Coast. Shelf Sci., 51, 773-787, 2000.

Dov Par, F.: Sooretama: The Atlantic rain forest of Brazil, SPB Academic Publishing, The Hague, 1992.

Doherty, R., Kutzbach, J., Foley, J., and Pollard, D.: Fully coupled climate/dynamical vegetation model simulations over northern Africa during the mid-Holocene, Clim. Dynaam., 16, 561-573, 2000.

Duivenvoorden, J. F. and Cleef, A. M.: Amazonian savanna vegetation on the sandstone near Araracuara, Colombia, Phytocoenesis, 24, 197-232, 1994.

Edwards, M. E., Anderson, P. M., Brubaker, L. B., Ager, T., Andreev, A. A., Bigelow, N. H., Cwynar, L. C., Eisner, W. R., Harrison, S. P., Hu, F.-S., Jolly, D., Lozhkin, A. V., MacDonald, G. M., Mock, C. J., Ritchie, J. C., Sher, A. V., Spear, R. W., Williams, J., and Yu, G.: Pollen-based biomes for Beringia 18,000, 6000 and $0{ }^{14}$ C yr B.P, J. Biog., 27, 521-554, 2000.

Eidt, R. C.: The climatology of South America, in: Biogeography and ecology in South America, edited by: Fittkau, E. J., 1, 54-81, Junk, The Hague, 1968.

Eiten, G.: The cerrado vegetation of Brazil, Bot. Rev., 2, 210-341, 1972.

Elenga, H., Peyron, O., Bonnefille, R., Prentice, I. C., Jolly, D., Cheddadi, R., Guiot, J., Andrieu, V., Bottema, S., Buchet, G., de Beaulieu, J. L., Hamilton, A. C., Maley, J., Marchant, R., Perez-Obiol, R., Reille, M., Riollet, G., Scott, L., Straka, H., Taylor, D., Van Campo, E., Vincens, A., Laarif, F., and Jonson, H.: Pollen-based reconstruction for southern Europe and Africa 18,000 years ago, J. Biog., 27, 621-634, 2000.

Ellis, E. C. and Ramankutty, N.: Putting people in the map: anthropgenic biomes of the world, Front Ecol. Environ., 6, 18-25, 2008.

Farrera, I., Harrison, S. P., Prentice, I. C., Ramstein, G., Guiot, J., Bartlein, P. J., Bonnefille, R., Bush, M., Cramer, W., von Grafenstein, U., Holmgren, K., Hooghiemstra, H., Hope, G., Jolly, D., Lauritzen, S. E., Ono, Y., Pinot, S., Stute, M., and Gu, Y.: Tropical climates at the last glacial maximum: a new synthesis of terrestrial palaeoclimate data. I. Vegetation, lake-levels and geochemistry, Clim. Dynam., 11, 823-856, 1999.

Flenley, J. R.: Tropical forests under the climates of the last 30,000 years, Climate Change, 39, 177-197, 1998.

Flenley, J. R. and King, S. M.: Late Quaternary pollen records from Easter Island, Nature, 307, 47-50, 1984.

Flenley, J. R., King, S. M., Jackson, J., Chew, C., Teller, J. T., and Prentice, M. E.: The late Quaternary vegetation and climatic history of Easter Island, J. Quat. Sci., 6, 85-115, 1991.

Fjeldså, J. A.: Un analisis biogeografico de la avifauna de los bosques de quenoa (Polylepis) de los Andes y su relevancia para establecer prioridades de conservacion, Memorias del Museo de Historia Natural, U.N.M.S.M. (Lima), 21, 207-221, 1992.

Fjeldså, J. A.: The avifauna of the Polylepis woodlands of the Andean highlands: the efficiency of basing conservation priorities on patterns of endemism, Bird Conservation International, 3, 3 55, 1993.

Fjeldså, J. A., Ehrlich, D., Lambin, E., and Prins, E.: Are biodiversity 'hot spots' correlated with current ecoclimatic stability? A pilot study using the NOAA-AVHRR remote sensing data, Biodiv. Conserv., 6, 399-420, 1997.

Foley, J. A., Prentice, I. C., Ramankutty, N., Levis, S., Pollard, D., Sitch, S., and Haxeltine, A.: An integrated biosphere model of land surface processes, terrestrial carbon balance and vegetation dynamics, Global Biogeochem. Cycles, 10, 603-628, 1996.

Gilli, A., Anselmetti, F. S., Ariztegui, D., Beres, M., McKenzie, J. A., and Markgraf, V.: Seismic stratigraphy, buried beach ridges and contourite drifts: the Late Quaternary history of the closed Lago Cardiel basin, Argentina ( $\left.49^{\circ} \mathrm{S}\right)$, Sedimentology, 52, 1-22, 2004.

Gentry, A. H.: A field guide to the families and genera of woody plants of South America (Colombia, Ecuador, Perú), Conservation International, Washington, D.C., 1993.

Godinez-Domínquez, M., Rojo-Vazquez, J. R., Galvan-Pina, V., and Aguilar-Palomino, B.: Changes in the structure of a coastal fish assemblage exploited by a small scale gillnet fishery during an El Niño-La Niña event, Est. Coast. Shelf Sci., 51, 773-787, 2000 . 
Godley, E. J. and Moar, N. T.: Vegetation and pollen analysis of two bogs on Chiloé, New Zea. J. Bot., 11, 255-268, 1973.

Gnécco, C.: An archaeological perspective on the Pleistocene/Holocene boundary in northern South America, Quatern. Int., 53, 3-9, 1999.

Gnécco, C. and Mohammed, A.: Technologia de cazadoresrecolectores subandinos: Analisis functional y organización tecnologica, Rev. of Colombian Anthropol., 31, 6-31, 1994.

Gnécco, C. and Mora, S.: Early occupations of the tropical forests of northern South America by hunter-gatherers, Antiquity, 71, 683-690, 1997.

Grabandt, R. J.: Pollen rain in relation to arboreal vegetation in the Colombian Cordillera Oriental, Rev. Palaeobot. Palynol., 29, 65-147, 1980.

Graf, K.: Palinologiadel cuaternario reciente en los Andes del Ecuador, del Peru, y de Bolivia, Boletin Servicio Geologico Bolivia, 4, 69-91, 1989.

Graf, K.: Pollen diagramme aus den Anden: Eine Synthese zur Klimageschichte und Vegetationsentwicklung seit der letzen Eiszeit. Physiche Geographie vol 34 University of Zurich, Switzerland, 72-94, 1992.

Graherr, G.: Farbatlas Ökosysteme der Erde: natürliche, naturnahe bund künstliche Land-Ökosysteme aus geobotanischer Sicht, Ulmer, Stuttgart, Germany, 1997.

Hansen, B. C. S. and Rodbell, D. T.: A glacial/Holocene pollen record from the eastern Andes of northern Peru, Quat. Res., 44, 216-227, 1995.

Hansen, B. C. S., Seltzer, G. O., and Wright Jr., H. E.: Late Quaternary vegetational change in the central Peruvian Andes, Palaeogeog., Palaeoclim., Palaeoecol., 109, 263-285, 1994.

Haberle, S. and Maslin, M.: Late Quaternary vegetation and climate change in the Amazon Basin based on a 50,000 year pollen record from the Amazon fan, ODP site 932, Quatern. Res., 51, 27-38, 1999.

Harley, R. M.: Introduction to the vegetation, in: Flora of the Pico das Almas. Chapada Diamantina, Bahia, Brazil, edited by: Stannards, B. L., Royal Botanic Gardens, Kew, 1995.

Harrison, S. P., Kutzbach, J. E., Liu, Z., Bartlein, P. J., OttoBliesner, B., Muhs, D., Prentice, I. C., and Thompson, R. S.: Mid-Holocene climates of the Americas: a dynamical response to changed seasonality, Clim. Dynam., 20, 663-668, 2003.

Haxeltine, A. and Prentice, I. C.: BIOME3: an equilibrium terrestrial biosphere model based on ecophysiological constraints, resource availability, and competition among plant functional types, Global Biogeochem. Cycles, 10, 693-709, 1996.

Heine, K. K.: Late Quaternary glacial sequences in Bolivia, Ecuador and México-data and palaeoclimatic implications. Abstracts, 14 th INQUA Congress, Berlin, 1995.

Heine, K. K.: Tropical South America during the Last Glacial maximum: evidence from glacial, periglacial and fluvial records, Quatern. Int., 72, 7-21, 2000.

Helmens, K. F. and Kuhry, P.: Middle and late Quaternary vegetational and climatic history of the Páramo de Agua Blanca (Eastern Cordillera Colombia), Palaeogeog., Palaeoclim., Palaeoecol., 56, 291-335, 1986.

Heusser, C. J.: Quaternary palynology of Chile, Quaternary of South America and the Antarctic Peninsula, 1, 5-22, 1982.

Heusser, C. J.: Three late Quaternary pollen diagrams from Southern Patagonia and their palaeoecological implications, Palaeo- geog., Palaeoclim., Palaeoecol., 118, 1-24, 1995.

Heusser, C. J., Denton, G. H., Hauser, A., Anderson, B., and Lowell, T.: Quaternary pollen records from the Archipelago de Chiloe in the context of glaciation and climate, Revista Geologica de Chile, 22, 25-46, 1995.

Heusser, C. J., Lowell, T., Heusser, L., Moreira, A., and Moreira, S. M.: Pollen sequence from the Llanquihue glaciation in marine Oxygen Isotope Stage 4-2, J. Quat. Sci., 15, 115-125, 2000.

Hollis, J. H. T. and Schilling, D. H.: Late Wisconsin-Weichselian mountain glaciers and small ice caps, in: The last great ice sheets, edited by: Denton, G. H. and Hughes, T. J., Wiley, New York, 1981.

Hooghiemstra, H.: Vegetation and climate history of the high plain of Bogotá, Columbia: a continuous record of the last 3.5 million years, Ganter Verlag, Vadiz, 1984.

Hooghiemstra, H. and Cleef, A. M.: Development of Vegetational and Climatic Sequence of the Area of the High Plain of Bogotá, in: Vegetation and climate history of the high plain of Bogotá, Columbia: a continuous record of the last 3.5 million years, edited by: Hooghiemstra, H., Ganter Verlag, Vadiz, 1984.

Hooghiemstra, H., Cleef, A. M., Noldus, G. W., and Kappelle, M.: Upper Quaternary vegetation dynamics and palaeoeclimatology of the La Chonta bog area (Cordillera de Talamanca, Costa Rica), J. Quat. Sci., 7, 205-225, 1992.

Hooghiemstra, H. and van der Hammen, T.: Neogene and Quaternary development of the neotropical rain forest : the forest refugia hypothesis, and a literature overview, Earth Sci. Rev., 44, 147-103, 1998.

Horn, S. P.: Prehistoric fires in the highlands of Costa Rica: sedimentary charcoal evidence, Rev. Bio. Trop., 37, 139-148, 1989.

Horn, S. P.: Postglacial vegetation and fire history in the Chirripó Páramo of Costa Rica, Quatern. Res., 40, 107-116, 1993.

Hück, K.: Die waldgeographische Regionen und Unterregionen von Südamerika (with map 1:55,000,000), Geographische Taschenbuch, 1960.

Huntingford, C., Cox, P. M., and Lenton, T. M.: Contrasting response of a simple terrestrial ecosystem model to global change, Ecol. Mod., 134, 41-58, 2000.

International Plant Names Project: International Plant Names Index, http://www.ipni.org, 2009.

Iselbe, G. and Hooghiemstra, H.: Recent pollen spectra of highland Guatemala, J. Biog., 22, 1091-1099, 1995.

Iselbe, G. and Hooghiemstra, H.: Vegetation and the Climate History of Montane Costa Rica since the Last Glacial, Quat. Sci. Rev., 16, 589-604, 1997.

Iselbe, G., Hooghiemstra, H., and van der Borg, K.: A cooling event during the Younger Dryas Chron in Costa Rica, Palaeogeog., Palaeoclim., Palaeoecol., 117, 73-80, 1995a.

Iselbe, G., Hooghiemstra, H., and van der Borg, K.: The Younger Dryas in the Cordillera de Talamanca, Costa Rica, Geologie en Mijnbouw, 74, 281-283, 1995b.

Iselbe, G., Hooghiemstra, H., Brenner, M., Curtis, J. H., and Hodell, D. A.: A Holocene vegetation history from lowland Guatemala, The Holo., 6, 265-271, 1996.

Jolly, D., Harrison, S. P., Damnati, B., and Bonnefille, R.: Simulated climate and biomes of Africa during the Late Quaternary: comparisons with pollen and lake status data, Quat. Sci. Rev., 17, 629-657, 1998b.

Jolly, D. and Haxeltine, A.: Effect of low glacial atmospheric $\mathrm{CO}_{2}$ 
on tropical African montane vegetation, Science, 276, 786-788, 1997.

Jolly, D., Prentice, I. C., Bonnefille, R., Ballouche, A., Bengo, M., Brenac, P., Buchet, G., Burney, D., Cazet, J-P., Cheddadi, R., Edorh, T., Elenga, H., Elmoutaki, S., Guiot, J., Laarif, F., Lamb, H., Lezine, A.-M., Maley, J., Mbenza, M., Peryon, O., Reille, M., Reynaud-Farrera, I., Riollet, G., Ritchie, J. C., Roche, E., Scott, L., Ssemmanda, I., Straka, H., Umer, M., Van Campo, E., Vilimumbalo, S., Vincens, A., and Waller, M.: Biome reconstruction from pollen and plant macrofossil data for Africa and the Arabian peninsula at 0 and $6 \mathrm{ka}$, J. Biog., 25, 997-1005, 1998a.

Kahn, F. and de Granville, J. J.: Palms in the forest ecosystems of Amazonia, Springer Verlag, Germany, 1992.

Kappelle, M.: Structural and floristic differences between western Atlantic and moist Pacific montane Myrsine-Quercus forest in Costa Rica. Academic Press, 1992.

Kappelle, M.: Ecology of mature and recovering Talamancan Montane Quercus forests, Costa Rica, PhD Thesis, University of Amsterdam, The Netherlands, 1995.

Kershaw, A. P. and McGlone, M.: The Quaternary history of the southern conifers, in: Ecology of Southern Conifers, edited by: Enright, N. J. and Hill, R. S., Melbourne University Press, 1995.

Kewensis: Index Kewensis version 2.0, Developed by systems simulations Ltd, Oxford University Press.

Kubatzki, C. and Claussen, M.: Simulation of the global biogeophysical interactions during the Last Glacial Maximum, Clim. Dynam., 14, 461-471, 1997.

Kuhry, P.: A Paleobotanical and palynological study of Holocene peat from the El Bosque Mire, located in a volcanic area of the Cordillera Central of Colombia, 1988a.

Kuhry, P.: Palaeobotantical-palaeoecological studies of tropical high Andean peatbog sections (Cordillera Oriental, Colombia), Dissertationes Botanicae 116, J. Cramer, Berlin-Stuttgart, 1988.

Kuhry, P., Salomons, J. B., Riezebos, P. A., and Van der Hammen, T.: Paleoecologia de los ultimos 6.000 anos en el area de la Laguna de Otun-El Bosque. In T. van der Hammen, P.A. Perez, and P. Pinto (eds.) Studies on Tropical Andean Ecosystem, Vol 1: La Cordillera Central Colombiana transecto Parque Los Nevados, Cramer, Vaduz, 1983.

Latin American Pollen Database (LAPD): http://www.ncdc.noaa. gov/paleo/lapd.html, last access 4 November 2009.

Ledru, M.-P.: Modifications de la vegetation du Brazil entre la dernieier epoque glaciaire et línterglaciaire ectuel, Comptes Rendus de l'Academie des Sciences Paris, 314, 117-123, 1992.

Ledru, M.-P.: Late Quaternary environmental and climatic changes in Central Brazil, Quatern. Res., 39, 90-98, 1993.

Ledru, M.-P., Behling, H., Fournier, M. L., Martin, L., and Servant, M.: Localisation de la fôret d'Acaucaria du Brazil au cours de l'Holocène. Implications paleoclimatiques, C.R. Acadamie Science Paris, 317, 517-521, 1994.

Ledru, M.-P., Bertaux, J., and Sifeddine, A.: Absence of Last Glacial Maximum records in lowland tropical forests, Quatern. Res., 49, 233-237, 1998.

Ledru, M.-P, Corderio, R. C., Dominquez, J. M. L., Martin, L., Mourguiart, P., Sifeddine, A., and Turcq, B.: Late-glacial cooling in Amazonia inferred from pollen at Lagoa de Caco, northern Brazil, Quatern. Res., 55, 47-56, 2001.

Ledru, M.-P. and Lorscheitter, M. L.: Vegetation dynamics in southern and Central Brazil during the last 10,000 yr B.P, Rev.
Palaeobot. Palynol., 99, 131-142, 1998.

Ledru, M.-P., Soares Braga, P. I., Soubies, F., Fournier, M. L., Martin, L., Suguio, K., and Turcq, B.: The last 50,000 years in the Neotropics (southern Brazil): evolution of vegetation and climate, Palaeogeog., Palaeoclim., Palaeoecol., 123, 239-257, 1996.

Leemans, R. and Cramer, W. P.: The IISA database for mean monthly values of temperature, precipitation and cloudiness on a global terrestrial grid. Rep IIASA Research RR-91-18, International Institute for Applied Systems Analysis, Laxenburg, Austria, 1991.

Leyden, B. W.: Guatemalan forest synthesis after Pleistocene aridity. Proceedings of the National Academy of Sciences (USA), 81, 4856-4859, 1984.

Leyden, B. W.: Late Quaternary aridity and Holocene moisture fluctuations in the Lake Valencia Basin, Venezuela, Ecol., 66, 12791295, 1985.

Leyden, B. W.: Man and climate in Maya lowlands, Quatern. Res., 28, 407-414, 1987.

Leyden, B. W., Brenner, M., Hodell, D. A., and Curtis, J. H.: Late Pleistocene climate in the central American lowlands, in: American Geophysical Union Geophys. Mono., 78, edited by: Swar, P. K., Washington, D.C., USA, 1993.

Leyden, B. W., Brenner, M., Hodell, D. A., and Curtis, J. H.: Orbital and internal forcing of the climate on the Yucatán peninsula for the past ca 36 ka, Palaeogeog., Palaeoclim., Palaeoecol., 109, 193-210, 1994.

Leyden, B. W., Brenner, M., Whitmore, T., Curtis, J. H., Piperno, D. R., and Dahlin, B. H.: A record of long and short-term climatic variation from northwest Yucatán: Cenote San Jose Chulchaca, in: The managed mosaic: Ancient Maya agriculture and resource use, edited by: Fedick, S. L., University of Utah Press, USA, 1995.

Liu, K. and Colinvaux, P.: Forest changes in the Amazon Basin at the LGM, Nature, 318, 556-577, 1985.

Lozano-Garcial, M. S. and Ortega-Guerroero, B.: Palynological and magnetic susceptibility records of Lake Chalco, central México, Palaeogeog., Palaeoclim., Palaeoecol., 109, 177-191, 1994.

Lozano-Garcial, M. S., Ortega-Guerroero, B., CaberalleroMirandan, M., and Urrutia-Fucugauchi, F.: Late Holocene Paleoenvironments of Chalco Lake, Central México, Quatern. Res., 40, 332-342, 1993.

Lozano-Garcial, M. S. and Xelhuantzi-Lopéz, M. S.: Some problems in the late Quaternary pollen records of Central México: Basins of México and Zacapu, Quatern. Int., 43, 117-123, 1993

Lumley, S. H. and Switsur, R.: Late Quaternary of the Taitao Peninsula, southern Chile, J. Quatern. Sci., 8, 161-165, 1993.

Maberly, D. J.: The Plant Book: A portable dictionary of the higher plants, Cambridge University Press, 1993.

Mancini, M. V.: Recent pollen spectra from forest and steppe of South Argentina: a comparison with vegetation and climate data, Rev. Palaeobot. Palynol., 77, 129-142, 1993.

Marchant, R. A., Almeida, L., Behling, H., Berrio, J. C., Bush, M., Cleef, A., Duivenvoorden, J., Kappelle, M., De Oliveira, P.E., Hooghiemstra, H., Teixeira de Oliveira-Filho, A., Ledru, M-P., Lozano-García, M. S., Ludlow-Wiechers, B., Mancini, V., Paez, M., Markgraf, V., Prieto, A., Rangel, O., and Salgado-Labouriau, M. L.: Distribution and ecology of parent taxa of pollen lodged 
within the Latin American Pollen Database, Rev. Palaeobot. Palynol., 121, 1-75, 2002c.

Marchant, R. A., Behling, H., Berrio, J. C., Cleef, A., Duivenvoorden, J., van Geel, B, van der Hammen, T., Hooghiemstra, H., Kuhry, P., Melief, B. M., van Reenen, G., and Wille, M.: Late Holocene Colombian vegetation dynamics, Quat. Sci. Rev., 20, 1289-1308, 2001a.

Marchant, R. A., Behling, H., Berrio, J. C., Cleef, A., Duivenvoorden, J., van Geel, B, van der Hammen, T., Hooghiemstra, H., Kuhry, P., Melief, B. M., van Reenen, G., and Wille, M.: A reconstruction of Colombian biomes derived from modern pollen data along an altitude gradient, Rev. Palaeobot. Palynol., 117, 79-92, 2001b.

Marchant, R. A., Behling, H., Berrio, J. C., Cleef, A., Duivenvoorden, J., van Geel, B, van der Hammen, T., Hooghiemstra, H., Kuhry, P., Melief, B. M., van Reenen, G., and Wille, M.: Colombian vegetation derived from pollen data at $0,3000,6000,9000$, $12,000,15,000$ and 18,000 radiocarbon years before present, J. Quatern. Sci., 17, 113-129, 2002a.

Marchant, R. A., Behling, H., Berrío, J. C., Cleef, A., Duivenvoorden, J., van Geel, B., van der Hammen, T., Hooghiemstra, H., Kuhry, P., Melief, B. M., van Reenen, G., and Wille, M.: Mid to Late Holocene vegetation disturbance in Colombia - a regional reconstruction, Antiquity, 78, 828-838, 2004b.

Marchant, R. A., Berrío, J. C., Behling, H., Boom, A., and Hooghiemstra, H.: Colombian dry moist forest transitions in the Llanos Orientales - a comparison of model and pollenbased biome reconstructions, Palaeogeog., Palaeoclimatol., Palaeoecol., 234, 28-44, 2006.

Marchant, R. A., Boom, A., Behling, H., Berrío, J. C., van Geel, B., van der Hammen, T., Hooghiemstra, H., Kuhry, P., and Wille, M.: Colombian vegetation at the Late Glacial Maximum - a comparison of model and pollen-based biome reconstructions, J. Quat. Sci., 19, 721-732, 2004.

Marchant, R. A., Boom, A., and Hooghiemstra, H.: Pollen-based biome and $\delta^{13} \mathrm{C}$ reconstructions for the past 450,000 years from the Funza II core, Colombia - ecosystem relationships to late Quaternary $\mathrm{CO}_{2}$ change as detected in the Vostok ice core record, Palaeogeog., Palaeoclim., Palaeoecol., 177, 29-45, 2002b.

Marchant, R. A. and Hooghiemstra, H.: Rapid environmental change in tropical Africa and Latin America about 4000 years before present: a review, Earth Sci. Rev., 66, 217-260, 2004.

Marchant, R. A. and Hooghiemstra, H.: 'Letter to the Editor' Climate of East Africa $6000{ }^{14} \mathrm{C}$ Yr B.P. as inferred from pollen data, By Odile Peyron, Dominique Jolly, Raymonde Bonnefille, Annie Vincens and Joël Guiot, Quatern. Res., 56, 133-135, $2001 b$.

Marchant, R. A. and Taylor, D. M.: Numerical analysis of modern pollen spectra and in situ montane forest - implications for the interpretation of fossil pollen sequences from tropical Africa, New Phytol., 146, 505-515, 2000.

Markgraf, V.: Late and postglacial vegetational and paleoclimatic changes in sub-Antarctic, temperate, and arid environments in Argentina, Palynol., 7, 43-52, 1983.

Markgraf, V.: Late Pleistocene and Holocene vegetation history of temperate Argentina: Lago Morenito, Bariloche, Diss. Bot., 72, 235-254, 1984.

Markgraf, V.: Late Pleistocene faunal extinction in southern Patagonia, Science, 228, 1110-1112, 1985a.
Markgraf, V.: Paleoenvironmental history of the last 10,000 years in northwestern Argentina, Zentralblatt für Geologie und Paläontolgie, 11/12, 1739-1749, 1985b.

Markgraf, V.: Paleoenvironmental changes at the northern limit of the sub-Antarctic Nothofagus forest, latitude 37S, Argentina, Quatern. Res., 28, 119-129, 1987.

Markgraf, V.: Fells cave: 11,000 years of changes in paleoenvironments, fauna, and human occupation. In J. Hyslop (ed.) Travels in Archaeology in South Chile, University of Iowa, Iowa City, USA, 1988.

Markgraf, V.: Late Pleistocene/Holocene paleoclimates from subAntarctic latitudes, Antarctic Journal of the United States, 24, $1-2,1989$

Markgraf, V.: Palaeoclimates in Central and South America since 18,000 BP based on pollen and lake-level records, Quat. Sci. Rev., 8, 1-24, 1989.

Markgraf, V.: Climatic history of Central and Southern America since 18,000 yr B.P., in: Global Climates since the Last Glacial Maximum, edited by: Wright, H. E., Kutzbach, J. E., Webb III, T., Ruddiman, W. F., Street-Perrot, F. A., and Bartlein, P. J., University of Minnesota Press, USA, 1993.

Markgraf, V.: Younger Dryas in southern South America?, Boreas, 20, 63-69, 1991.

Markgraf, V.: Paleoenvironments and paleoclimates in Tierra del Fuego and southernmost Patagonia, South America. Palaeogeog., Palaeoclim., Palaeoecol. 102, 53-68, 1993.

Markgraf, V.: Past climates of South America, in: Climates of the Southern Continents: Present, Past and Future, edited by: Hobbs, E., Lindesay, J. A., and Bridgman, H. A., Wiley, Chichester, 1998.

Markgraf, V., Baumgartner, T. R., Bradbury, J. P., Diaz, H. F., Dunbar, R. B., Luckman, B. H., Seltzer, G. O., Swetman, T. W., and Villalba, R.: Paleoclimate reconstruction along the Pole-EquatorPole transect of the Americas (PEP 1), Quat. Sci. Rev., 19, 125$140,2000$.

Markgraf, V., Bradbury, J. P., and Fernández, P.: Bajada de Rahue, Province Neuquen, Argentina: an interstadial deposit in northern Patagonia, Palaeogeog., Palaeoclim., Palaeoecol., 56, 251-258, 1986.

Martin, L., Fournier, M., Mourguiart, P., Sifeddine, A., and Turcq, B.: Southern Oscillation signal in South American palaeoclimatic data of the last 7000 years, Quatern. Res., 39, 338-346, 1993.

Martin, L., Bertaux, J., Correge, T., Ledru, M.-P., Mourguiart, P., Sifeddine, A., Soubiès, F., Wirrmann, D., Suguio, K., and Turcq, B.: Astronomical forcing of contrasting rainfall changes in tropical South America between 12,400 and 8800 cal yr BP, Quatern. Res., 47, 117-122, 1997.

Mayle, F. E., Burbridge, R., and Killeen, T. J.: Millennial-scale dynamics of southern Amazonian rain forest, Science, 290, 22912294, 2000.

Melief, A. B. M.: Late Quaternary palaeoecology of the Parque National los Nevados Cordillera Central, and Sumapaz Cordillera Oriental areas, Colombia, Dissertation University of Amsterdam, Amsterdam, The Netherlands, 1985.

Mercer, J. H. and Ager, T. A.: Glacial and floral changes in southern Argentina since 14,000 years ago, National Geographic Science Reports, 15, 457-477, 1983.

Moreno, P. I.: Vegetation and climate near Lago Llanquihue in 
the Chilean Lake District between 20,200 and $950{ }^{14} \mathrm{C}$ yr BP, J. Quat. Sci., 12, 485-500, 1994.

Metclafe, S. E., O’Hara, S. L., Caballero, M., and Davies, S. J.: Records of late Pleistocene-Holocene climate change in México - a review, Quat. Sci. Rev., 19, 699-721, 2000.

Monsalve, J. G.: A pollen core from the Hacienda Lusitania, Pro Calima, 4, 40-44, 1985.

Mourguiart, P. H., Argollo, J., and Wirrmann, D.: Evoucioun del lago Titicaca desde 25,000 nos BP, in: Climas cuaternarios en America del Sur, edited by: Argollo, J. and Mourguiart, Ph., Editions ORSTOM-La Paz, Bolivia, p. 157-171, 1995.

Noblet-Docoudré, N., Claussen, M., and Prentice, C.: MidHolocene greening of the Sahara: first results of the GAIM 6000 year BP experiment with two asynchronously coupled atmosphere/biome models, Clim. Dynam., 16, 643-659, 2000.

Northrop, L. A. and Horn, S. P.: Precolombian agriculture and forest disturbance in Costa Rica: palaeoecological evidence from two lowland rain forest lakes, The Holo., 6, 289-299, 1996.

Ortega-Guerrero, B.: Paleomagnestismo, magnetoestrtifia y paleoecologia del Cuaternario tardio en el Lago de Chalco, Cuenca de México, Dissertation, National University of México, 1992.

Ortega-Rosas, C. I., Guiot, J., Peñalba, M. C., and Ortiz-Acosta, M. E.: Biomization and quantitative climate reconstruction techniques in northwestern Mexico - with an application to four Holocene pollen sequences, Glob. Plan. Cha., 61, 242-266, 2008a.

Ortega-Rosas, C. I., Peñalba, M. C., and Guiot, J.: Holocene altitudinal shifts in vegetation belts and environmental changes in the Sierra Madre Occidental, northwestern Mexico based on modern and fossil pollen data, Rev. Paleobot. Palynol., 151, 1-20, $2008 \mathrm{~b}$.

Páez, M. M. and Prieto, A. R.: Paleoenvironmental reconstruction by pollen analysis from loess sequences from the Southeast of Buenos Aires (Argentina), Quatern. Int., 17, 139-153, 1993.

Pendall, E., Markgraf, V., White, J. W. C., and Dreier, M.: Multiproxy record of late Pleistocene-Holocene climate and vegetation changes from a peat bog in Patagonia, Quatern. Res., 55, 168178, 2001.

Peng, C. H., Guiot, J., and van Campo, E.: Estimating changes in terrestrial vegetation and carbon storage: using palaeoecological data and models, Quatern. Sci. Rev., 17, 719-735, 1998.

Pereira, B. A. S., de-Mendonça, R. C., Filgueiras, T. S., de-Paula, J. E., and Heringer, E. P.: Levantamento florístico da área de proteção ambiental (APA) da bacia do Rio São Bartolomeu, Distrito Federal, Anais XXXVI Congresso Brazileiro de Botânica, Socied. Bot. Brazil, 1985, edited by: IBAMA, Curitiba, vol. 1, 1990.

Peyron, O., Jolly, D., Bonnefille, R., Vincens, A., and Guiot, J.: Climate of East Africa $6000{ }^{14} \mathrm{C}$ yr B.P. as inferred from pollen data, Quatern. Res., 54, 90-101, 2000.

Pickett, E. J., Harrison, S. P., Hope, G., Harle, K., Dodson, J. R., Kershaw, A. P., Prentice, I. C., Backhouse, J., Colhoun, E. A., D'Costa, D., Flenley, J., Grindrod, J., Haberle, S., Hassell, C., Kenyon, C., Macphail, M., Martin, H., Martin, A. C., McKenzie, M., Newsome, J. C., Penny, D., Powell, J., Raine, J. I., Southern, W., and Stevenson, J.: Pollen-based reconstructions of biome distributions for Australia, Southeast Asia and the Pacific (SEAPAC region) at 0, 6000 and 18,000 C-14 yr BP, J. Biogeog., 31, 1381-1444, 2004.

Piperno, D. R., Bush, M., and Colinvaux, P. A.: Palaeoenvironments and human occupation in Late Glacial Panama, Quatern. Res., 33, 108-116, 1990.

Piperno, D. R., Bush, M., and Colinvaux, P. A.: Palaeoecological perspectives on human adaption in Central Panama. I: The Pleistocene, Geoarchaeol., 6, 201-226, 1991a.

Piperno, D. R., Bush, M., and Colinvaux, P. A.: Palaeoecological perspectives on human adaption in Central Panama. II: The Holo., Geoarchaeol., 6, 227-250, 1991b.

Piperno, D. R., Ranere, A. J., Holst, I., and Hansell, P.: Starch grains reveal early root crop horticulture in the Panamanian tropical forest, Nature, 407, 894-897, 2000.

Pires, J. M. and Prance, G. T.: The vegetation types of the Brazilian Amazon, in: Key Environments Amazonia, edited by: Prance, G. T. and Lovejoy, T., Pergamon Press, 1985.

Prance, G. T.: The changing forest, in: Key Environments Amazonia, edited by: Prance, G. T. and Lovejoy, T., Pergamon Press, 1985.

Prentice, I. C., Cramer, W., Harrison, S. P., Leemans, R., Monserud, R. A., and Solomon, A. M.: A global biome model based on plant physiology and dominance, soil properties and climate, J. Biog., 19, 117-134, 1992.

Prentice, I. C., Sykes, M. T., Lautenschlager, M., Harrison, S. P., Denissenko, O., and Bartlein, P.: Modelling global vegetation patterns and terrestrial carbon storage at the last glacial maximum, Glob. Ecol. Biogeog. Lett., 3, 67-76, 1993.

Prentice, I. C., Guiot, J., Huntley, B., Jolly, D., and Cheddadi, R.: Reconstructing biomes from palaeoecological data: a general method and its application to European pollen data at 0 and $6 \mathrm{ka}$., Clim. Dynam., 12, 185-194, 1996a.

Prentice, I. C., Harrison, S. P., Jolly, D., and Guiot, J.: The climate and biomes of Europe at $6000 \mathrm{yr}$ BP: comparison of model simulations and pollen-based reconstructions, Quat. Sci. Rev., 17, 659-668, 1996b.

Prentice, I. C. and Webb III, T.: BIOME 6000: reconstructing global mid-Holocene vegetation patterns from palaeoecological records, J. Biog., 25, 997-1005, 1998.

Prentice, I. C., Jolly, D., and BIOME 6000 Participants: MidHolocene and glacial-maximum vegetation: geography of the northern continents and Africa, J. Biog., 27, 507-519, 2000.

Prieto, A. R.: Late Quaternary vegetation and climatic changes in the Pampa grassland of Argentina, Quatern. Res., 45, 73-88, 1996.

Prieto, A. R. and Páez, M. M.: Pollen analysis of discontinuous stratigraphical sequences: Holocene at Cerro La China locality (Buenos Aires, Argentina), Quaternary of South America and Antarctica Peninsula, 7, 219-236, 1989.

Rodgers, J. C. and Horn, S. P.: Modern pollen spectra from Costa Rica, Palaeogeog., Palaeoclim., Palaeoecol., 124, 53-71, 1996.

Rull, V.: A palynological record of a secondary succession after fire in Gran Sabana, Venezuela, J. Quat. Sci., 14, 137-152, 1999.

Rull, V., Salgado-Labouriau, M. L., Schubert, C., and Valastro Jr., S.: Late Holocene temperature depression in the Venezuelan Andes: Palynological evidence, Palaeogeog., Palaeoclim., Palaeoecol., 60, 109-121, 1987.

Rzedowski, V.: Vegetacion de México, Editorial Lémusa, México, 1983.

Salami, M.: Additional information on the findings in the Mylodo cave at Ultima Esperanza, Acta Geografica, 14, 314-333, 1955.

Salgado-Labouriau, M. L.: Sequence of colonisation by plants in 
the Venezuelan Andes after the Pleistocene glaciation, J. Palynol., 23-24, 189-204, 1988.

Salgado-Labouriau, M. L.: Late Quaternary palaeoclimate in the savannas of South America, J. Quatern. Sci., 12, 371-379, 1997.

Salgado-Labouriau, M. L., Barberi, M., Ferraz-Vicentini, K. R., and Parizzi, M. G.: A dry climatic event during the late Quaternary of tropical Brazil, Rev. Palaeobot. Palynol., 99, 115-129, 1998.

Salgado-Labouriau, M., Ferraz-Vicentini, K. R., and Parizzi, M. G.: A dry climatic event during the late Quaternary of tropical Brazil, Rev. Palaeobot. Palynol., 99, 1515-129, 1998.

Saporito, M. S.: Chemical and mineral studies of a core from Lake Patzcuaro, México, MSc thesis, University of Minnesota, Minneapolis, Minnesota, USA, 1975.

Sarmiento, G.: The dry plant formations of South America and their floristic connections, J. Biog., 2, 233-251, 1975.

Schäbitz, F.: Untersuchungen zum aktuellen Pollenniederschlag und zur Holozänen Klima-und Vegetationsentwicklung in den Anden Nord-Neuquéns, Argentinien, Bamberger Geographische Schriften, 8, 1-131, 1989.

Schäbitz, F.: Holocene vegetation and climate in southern Santa Cruz, Argentina, Bamberger Geographische Schriften [Bd.] 11, 235-244, 1991.

Schäbitz, F.: Holocene climatic variations in northern Patagonia, Argentina, Palaeogeog., Palaeoclim., Palaeoecol., 109, 287-294, 1994.

Schreve-Brinkman, E. J.: A palynological study of the upper Quaternary sequence in the El Abra corridor and rock shelters Colombia, Palaeogeog., Palaeoclim., Palaeoecol., 25, 1-109, 1978.

Schmithüsen, J.: Atlas zur Biogeographie, Bibliographisches Institut, Mannheim/Wien/Zürich, 1976.

Schofield, K.: Plants of the Galapagos Islands, Universe Books, 1984.

Seibert, P.: Farbatlas Südamerika - Landschaften und Vegetation, Verlag Eugen Ulmer, Mannheim, 1996.

Seltzer, G. O.: Recent glacial history and palaeoclimate of the Peruvian-Bolivian Andes, Quatern. Sci. Rev., 9, 137-152, 1990.

Servant, M., Maley, J., Turcq, B., Absy, M., Brenac, P. Furnier, M., and Ledru, M.-P.: Tropical Forest changes during the late Quaternary in African and South American lowlands, Glob. Planet. Cha., 7, 25-40, 1993.

Sifeddine, A., Martin, L., Turcq, B., Volkermer-Ribero, C., Soubies, F., Cordeiro, R. C., and Suguio, K.: Variations in the Amazonian rain forest environment: a sedimentological record covering 30,000 years, Palaeogeog., Palaeoclim., Palaeoecol., 168, 221-235, 2001.

Steege, H. T.: The use of forest inventory data for a National Protected Area Strategy in Guyana, Biodiv. Conserv., 7, 1457-1483, 1998.

Takahara, H., Sugita, S., Harrison, S. P., Miyoshi, N., Morita, Y., and Uchiyama, T.: Pollen-Based Reconstruction of Japanese Biomes at 0, 6000 and 18,000 yr B.P., J. Biog., 27, 665-683, 2001.

Tarasov, P. E., Webb III, T., Andreev, A. A., Afanas'eva, N. B., Berezina, N. A., Bezusko, L. G., Blyakharchuk, T. A., Bolikhovskaya, N. S., Chernavskaya, M. M., Chernova, G. M., Dorofeyuk, N. I., Dirksen, V. G., Elina, G. A., Filimonova, L. V., Glebov, F. Z., Guiot, J., Gunova, V. S., Harrison, S. P., Jolly, D., Khomutova, V. I., Kvavadze, E. V., Osipova, I. M., Panova, N.
K., Prentice, I. C., Saarse, L., Sevastyanov, C. S., Volkova, V. S., and Zernitskaya, V. P.: Present day and mid-Holocene Biomes Reconstructed from Pollen and Plant Macrofossil Data from the Former Soviet Union and Mongolia, J. Biog., 25, 1029-1053, 1998a.

Tarasov, P. E., Cheddadi, R. Guiot, J., Sytze, B., Peyron, O., Belmonte, J., Ruiz-Sanchez, V, Saadi, F., and Brewer, S.: A method to determine warm and cool steppe biomes from pollen data; application to the Mediterranean and Kazakhstan regions, J. Quatern. Sci., 13, 335-344, 1998b.

Texier, D., de Noblet, N., Harrison, S. P., Haxeltine, A., Joussaume, S., Jolly, D., Laarif, F., Prentice, I. C., and Tarasov, P. E.: Quantifying the role of biosphere-atmosphere feedbacks in climate change: a coupled model simulation for $6000 \mathrm{yr}$ B.P. and comparison with palaeodata for Northern Eurasia and northern Africa, Clim. Dynam., 13, 865-881, 1997.

Thompson, L. G., Mosely-Thompson, E., Davis, E. M., Lin, P. E., Henderson, K. A., Cole-Dai, B., and Liu, K.: Late glacial stage and Holocene tropical ice core records from Huascaren, Peru, Science, 269, 46-50, 1995.

Thompson, R. S. and Anderson, K. H.: Biomes of Western North America at 18,000, 6,000 and $0{ }^{14} \mathrm{C}$ yr B.P. Reconstructed from Pollen and Packrat Midden Data, J. Biog., 27, 655-664, 2000.

Thouret, J., van der Hammen, T., Salomons, B., and Juvigne, E.: Late Quaternary glacial studies in the Cordillera Central, Colombia, based on glacial geomorphology, tephra-soil stratigraphy, palynology and radiocarbon dating, J. Quatern. Sci., 12, 347369, 1997.

Thomas, M. F.: Late Quaternary environmental changes and the alluvial record in humid tropical environments, Quatern. Int., 72, 23-36, 2000.

Tuomisto, H. and Ruokolainen, K.: The role of ecological knowledge in explaining biogeography, and biodiversity in Amazonia, Biodiv. Conserv., 6, 347-357, 1997.

Valencio, A. A., Creer, K. M., Sinito, A. M., Mazzoni, M. M., Alonso, M. S., and Markgraf, V.: Palaeomagnetism, sedimentology, radiocarbon age determinations and palynology of the Llao area, southwestern Argentina (lat. $41 \mathrm{~S}$, long. $71.3 \mathrm{~W}$ ): paleolimnological aspects, Quaternary of South America and Antarctic Peninsular., 3, 109-148, 1985.

Van der Hammen, T.: Palinología de la region de Laguna de los Bobos, Historia de su clima, vegetacion y agricultura durante los últimos 5000 anos. Revista Acad Colombiana Ciencias Exactas Fisicas Naturales, 11, 359-361, 1962.

Van der Hammen, T.: A Palynological Study on the Quaternary of British Guyana, Leidse Geologische Mededelingen, 29, 126168, 1963.

Van der Hammen, T.: Changes in vegetation and climate in the Amazon Basin and surrounding areas during the Pleistocene, Geologie en Mijnbouw, 51, 641-643, 1972.

Van der Hammen, T.: The Pleistocene changes of vegetation and climate in tropical South America, J. Biog., 1, 3-26, 1974.

Van der Hammen, T. and Absy, M.: Amazonia during the last glacial Palaeogeog., Palaeoclim., Palaeoecol., 109, 247-261, 1994.

Van der Hammen, T., Barelds, T. J., de Jong, H., and de Veer, A. A.: Glacial sequence and environmental history in the Sierra Nevada del Cocuy Colombia, Palaeogeog., Palaeoclim., Palaeoecol., 32, 247-340, 1980. 
Van der Hammen, T. and González, E.: Upper Pleistocene and Holocene climate and vegetation of the Saban de Bogotá, Leides Geologische Mededelingen, 25, 261-315, 1960.

Van der Hammen, T. and González, E.: A Late glacial and Holocene pollen diagram from Cienaga del Vistador Dep. Boyaca, Colombia, Leids Geologische Mededelingen, 32, 193-201, 1965.

Van Geel, B. and Van der Hammen, T.: Upper Quaternary vegetation and climatic sequence of the Fuquene area Eastern Cordillera, Colombia, Palaeogeog., Palaeoclim., Palaeoecol., 14, 9-92, 1973.

Valdes, P.: South American palaeoclimate model simulations: how reliable are the models, J. Quat. Sci., 15, 357-368, 2000.

Veblen, T. T., Burns, B. R., Kitzberger, T., Lara, A., and Villalba, R.: The Ecology of the Conifers of southern South America, in: Ecology of southern Conifers, edited by: Enright, N. J. and Hill, R. S., Melbourne University Press, 1995.

Vernet J.-L., Wengler, L., Solari, M.-E., Ceccantini, G., Fournier, M., Fournier, M., Ledru, M.-P., and Soubies, F.: Feux, climats et végétations au Brésil central durant l'Holocène: les donnees dún profil de sol a charbons de bois (Salitre, Minas Gerais), CRAS, Paris, 319, 1391-1397, 1994.

Villagran, C.: Expansion of Megallanic Moorland during the Late Pleistocene: palynological evidence from Northern Isla de Chiloé, Chile, Quatern. Res., 30, 304-314, 1988.

Watts, W. A. and Bradbury, J. P.: Palaeoecological studies on Lake Patzcuaro on the wets-central Mexican Plateau and at Chalco in the Basin of México, Quatern. Res., 17, 56-70, 1982.

Webb III, T.: A global paleoclimatic data base for $6000 \mathrm{yr}$ BP, DOE/EV/10097-6, US Department of Energy, Washington, 1985.

Wijmstra, T. A. and van der Hammen, T.: Palynological data on the history of tropical savannas in northern South America, Leides Geologische Mededelingen, 38, 71-90, 1966.

Wille, M., Hooghiemstra, H., Behling, H., Van der Borg, K., and Negret, A. J.: Environmental change in the Colombian subandean forest belt from 8 pollen records: the last $50 \mathrm{kyr}$, Veg. His. Archaeobot., 10, 61-77, 2001.

Wille, M., Negret, J. A., and Hooghiemstra, H.: Paleoenvironmental history of the Popayan area since $27000 \mathrm{yr}$ BP at Timbio, Southern Colombia, Rev. Palaeobot. Palynol., 109, 45-63, 2000.

Wijmstra, T. A. and van der Hammen, T.: Palynological data on the history of tropical savannas in northern South America, Leidse Geologische Mededelingen, 38, 71-90, 1966.

Wijmstra, T. A.: The Palynology of the Guiana coastal Basin, University of Amsterdam, 72 pp, 1971.
Whitmore, T. C.: Quaternary History of tropical America, in: Biogeography and Quaternary History in Tropical America, edited by: Whitmore, T. C. and Prance, G. T., Clarendon Press, Oxford, 1987.

Williams, J. W., Summer, R. S., and Webb III, T.: Applying Plant Functional types to construct biome maps from eastern North American Pollen Data: Comparisons with model results, Quat. Sci. Rev., 17, 607-627, 1998.

Williams, J. W., Webb III, T., Richard, P. J. H., and Newby, P.: Late Quaternary Biomes of Canada and the Eastern United States, J. Biog., 27, 523-538, 2000.

Wingenroth, M. and Suarez, J.: Flores de los Andes - Alta montaña de Mendoza. IANIGLA (Instituto Argentino de Nivolgia y Glacologia, Argentina, 1984.

Witte, H. J. L.: Present and past vegetation and climate in the North Andes (Cordillera Central, Colombia); a quantitative approach. $\mathrm{PhD}$ Thesis, University of Amsterdam, The Netherlands, 1994.

Woodward, F. I.: Climate and plant distribution, Cambridge University Press, Cambridge, 1987.

Wu, H., Guiot, J., Brewer, S., and Guo, Z.: Climatic changes in Eurasia and Africa at the last glacial maximum and midHolocene: reconstruction from pollen data using inverse vegetation modelling, Clim. Dynam., 29, 211-229, 2007 a.

Wu, H., Guiot, J., Brewer S., Guo, Z., and Peng, C.: Dominant factors controlling glacial and interglacial variations in the treeline elevation in tropical Africa, PNAS 104, 9720-9724, 2007b

Yu, G. and Harrison, S. P.: Lake status records from Europe: data base documentation. NOAA Paleoclimatology Publication Series, report, 3, 1-451, NOAA, Boulder, Colorado, 1995.

Yu, G., Prentice, I. C., Harrison, S. P., and Sun, X.: Pollen-based biome reconstruction for China at 0 and 6000 years, J. Biog., 25, 1055-1069, 1998.

Yu, G., Xudong, C., Jian, N., Cheddadi, R., Guiot, J., Huiyou, H., Harrison, S. P., Ci-xuan, H., Jolly, D., Manhong, K., Zhaochen, K., Shengfeng, L., Wen-yi, L., Liew, P. M., Gunagxu, L., Jinling, L., Liu, K-B., Prentice, I. C., Guoyu, R., Changqing, S., Sugita, S., Xiangjun, S., Lingyu, T., Van Campo, E., Yumei, X., Qinghai, X., Shun, Y., Xiangdong, Y., and Zhuo, Z.: Palaeovegetation of China: a pollen data-based synthesis for the mid-Holocene and last glacial maximum, J. Biog., 27, 635-664, 2001.

Xelhuantzi-López, M. S.: Palynologie et paléoenvironment du bassin de Zacapu, Michoancán, Mexique, depuis 8000 ans, Geofisica Internacional., 34, 239-248, 1995. 